\title{
Contribution to the modelling of the light field distribution within Synechocystis sp. PCC 6803 cultures and its influence on cellular photosynthesis processes
}

PhD dissertation by:

David Fuente Herraiz

Supervisors:

Dr. Javier Fermín Urchueguía Schölzel

Dr. Pedro Fernández de Córdoba

Dr. Sascha Rexroth

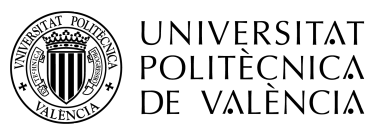

Departament de Matemàtica Aplicada

Universitat Politècnica de València

May, 2018 



\section{Acknowledgements}

La presente tesis engloba buena parte del trabajo realizado durante los últimos años y cierra mi primera etapa como investigador, vocación profesional que tengo desde hace muchos años. En este sentido, todas las personas que de alguna manera me han ayudado a conseguir mi objetivo, tienen mi profundo agradecimiento.

Querría agradecer todo el apoyo y comprensión que me han brindado mis tutores, Javier, Pedro y Sascha, tanto en los momentos buenos, como en los complicados. Muchas reuniones a última hora en las que discutíamos cómo mejorar el trabajo ya realizado, siempre con pasión y desde la humildad. Sin ellos, seguramente no estaría aquí. Además, han conseguido que aprenda conceptos hasta ahora desconocidos para mí, especialmente en el área de la óptica. También agradezco la oportunidad que me dieron al poder participar en un proyecto europeo que contó con tantos investigadores de renombre como fue CyanoFactory.

Si alguien se merece una mención especial a la persona con mejor sentido del humor y más generosidad, ese es sin duda Alberto Conejero, que en muchas ocasiones se ha comportado prácticamente como si fuera un director de tesis más.

Por otro lado, durante mis primeros años y mientras perfilaba mi investigación, hubo varios investigadores que me dedicaron su tiempo. Quiero destacar a Daniel, Ramón y Arnau, de los cuales también aprendí mucho. Especialmente me quiero acordar del apoyo de Daniel, que sin disponer casi de tiempo libre, siempre sacaba un hueco para atenderme. 
Otra persona de la cual he aprendido mucho es Maria Siurana, una brillante trabajadora con la que he vivido muchas aventuras a nivel técnico, docente y burocrático. Tampoco puedo olvidarme de Joan Vázquez, persona comprometida con los demás, que también ha aportado su granito de arena con sus sugerencias y sabios consejos. Asimismo recordar a mis compañeros de despacho, Miguel, Víctor y Borja, con los que convivido felizmente durante mucho tiempo. Especialmente con Borja, a quien conozco desde hace muchos años y ha demostrado ser una persona noble, y colaborativa. Por supuesto, también me acuerdo de aquellos estudiantes que estuvieron en nuestro laboratorio aprendiendo aspectos relacionados de la fotosíntesis, pero que también contribuyeron al desarrollo del equipo: Cristina, Katharina, Melanie y Carlos.

Hay otras personas que, de alguna manera, han sido parte de estos últimos años de desarrollo personal y profesional. Quiero recordar a Vicente Boria, con el cual intentamos una propuesta de astrobiología y, aunque finalmente la propuesta no cuajó, me sirvió para introducirme en dicho campo de trabajo, que quien sabe, quizás pueda retomar en el futuro. También mencionar a todos los estudiantes e investigadores de los distintos equipos iGEM en los que he participado y que de alguna manera han complementado mi formación.

Respecto a los amigos que han estado ahí, también quiero dar las gracias a Raquel por asesorarme en la redacción de proyectos, a Ivana por haberme ayudado durante la fase de redacción de la tesis y a Carolina por solucionarme problemas con la implementación del manuscrito. También a Veronica por darme algún consejo idiomático.

No me puedo olvidar del cariño de mis padres y de mi hermano, siempre en las duras y en las maduras, facilitándome las cosas cuando mi tiempo escaseaba y animando a continuar con la investigación.

Si hay alguna persona que considera que ha contribuido a mi investigación y no está aquí mencionada, le pido sinceras disculpas y le otorgo la posibilidad de reclamarme una cerveza como compensación. 
My big gratitude to Joseph Keller, Matthias Rögner and Carlos Lizama as they are another fundamental part of the here presented research contributions.

I would also like to thank Edda Klipp for hosting me in her lab. I found there a cohort of young talented researchers who deliver incredible results in a fantastic work atmosphere. Among the different students who helped me, Heiske Margit deserves a special mention. Sometimes you can feel after few minutes of conversation who is a really generous person. Without knowing me, she shared with me a big part of her knowledge in electron transport chain modelling that I will hopefully apply in my future research.

Regarding other reseach stages abroad, I want to highlight my short stage at Giuseppe's Torzillo group in Firenze. Giuseppe, Bernardo and Eleftherios were a fantastic host team, who deliver pioneer results in the field of photobioreactors and with whom I will hopefully keep in touch in the future.

The dozens of researchers that have sent to me unreachable published manuscripts, data and knowledge, and thus have contributed to my learning experience, deserve also a mention, especially Dariusz Stramski and David Lea-Smith.

After so many years of research, I cannot come up with a full list of all the people who had helped me, but in any case I want to offer my sincere thanks to all of them. 



\section{Abstract}

The present doctoral thesis, entitled "Contribution to the modelling of the light field distribution within Synechocystis sp PCC 6803 cultures and its influence on cellular photosynthesis processes", includes several works whose objective is to advance in the understanding of the light distribution in cyanobacterial cultures and in the effects of light on the photosynthetic mechanisms of these microorganisms. It is, ultimately, another step towards the integration of mathematical models on photosynthesis at the cellular level and at the scale of culture. First, to understand how a culture of photosynthetic bacteria behaves, it is essential to predict the distribution of the light field along the bioreactor profile, both at the level of total intensity, and with respect to its photon flux distribution. The distribution of wavelengths present in the medium is important since many processes of photosynthesis are regulated by certain wavelengths and are therefore modulated by the spectral distribution - the colour - of the light. In this sense, although there are several methodologies that predict the spatial evolution of the light field, these approaches usually consist in calculations that provide a non-analytical solution and are computationally demanding. Therefore, to have a simple tool and in turn scalable with other models of photosynthetic processes, would allow to advance in the understanding of them. Taking advantage of the inherent optical properties of the culture, a mathematical model based on the self-consistent field concept was developed. This algorithm, named in the corresponding publication as Auto-consistent Field Approximation Algorithm (AFA), provides an estimation of the light field, including the spectral evolution thereof along the optical path-length, for acclimated cultures to different radiation values. This research was published in the journal Algal Research through the article entitled "Light distribution and spectral composition within cultures of micro-algae: Quantitative modelling of the light field in photobioreactors", in which the algorithm is validated with experimental data of two strains of study of the cyanobacterium Synechocystis. Although the results were satisfactory, the use of the Lambert-Beer 
Law with a constant attenuation value, cannot correctly model the part of the light field with less intensity, where the attenuation coefficient ceases to be constant and the behaviour deviates from the exponential. Therefore, it was decided to model the light field with a function that generalizes the exponential case through the use of fractional calculus. A Mittag-Leffler function was used that fulfilled the formal requirements and offered a better data fit than that obtained with the LambertBeer law. As a remarkable finding, it was determined that the value of this parameter, which characterises the Mittag-Leffler function, was the same for the empirical data of both studied strains. This work was published in the contribution called "Estimation of the light field in photosynthetic microorganism cultures through Mittag-Leffler functions at depleted light conditions" in the journal Journal of Quantitative Spectroscopy $\mathcal{E} R a$ diative Transfer. Thereafter we proceeded to use both research works to calculate the light field within Synechocystis cultures and relate it to its maximum productivity. Specifically, it has been studied, as an indicator of the performance of photosynthesis, the production of oxygen and the associated respiratory mechanisms under different light intensities. This research is in its final phase and the writing of the article is being finalised to submit it to a scientific journal soon. This manuscript is titled "Experimental characterization of Synechocystis sp. PCC 6803 cultures productivity up on light conditions". Finally, a fourth contribution entitled "Individual pigment contribution to overall in vivo absorption in Synechocystis sp. PCC 6803 cells" is under development. This research studies the amount of light absorbed by Synechocystis chromophores according to the type of employed illumination and calculates the concentration of pigments present in the cell. In summary, the thesis presents modelling tools to the field of photosynthesis in order to advance in the integration of mathematical models that relate optical aspects with biological phenomena and thus support biotechnological initiatives in the field of cyanobacteria cultivation. 


\section{Resumen}

La presente tesis doctoral, titulada "Contribution to the modelling of the light field distribution within Synechocystis sp. PCC 6803 cultures and its influence on cellular photosynthesis processes", engloba diversos trabajos cuyo objetivo es avanzar en la compresión de la distribución lumínica en cultivos de cianobacterias y en los efectos de la luz sobre los mecanismos fotosintéticos de dichos microorganismos. Se trata, en definitiva, de otro paso hacia la integración de modelos matemáticos sobre la fotosíntesis a nivel celular y a escala de cultivo. En primer lugar, para comprender cómo se comporta un cultivo de bacterias fotosintéticas, es fundamental predecir la distribución del campo de luz a lo largo del perfil del biorreactor, tanto a nivel de intensidad total, como respecto a su distribución de flujo de fotones. La distribución de longitudes de onda presente en el medio es importante puesto que muchos procesos de la fotosíntesis están regulados por ciertas longitudes de onda y, por tanto, están modulados por la distribución espectral - el color - de la luz. En este sentido, si bien existen diversas metodologías que predicen la evolución espacial del campo de luz, dichas aproximaciones suelen consistir en cálculos que aportan una solución no analítica y con una carga computacional elevada. Por ello, poder disponer de una herramienta sencilla y a su vez escalable con otros modelos de procesos fotosintéticos, permitiría avanzar en el entendimiento de los mismos. Aprovechando las propiedades inherentes ópticas del cultivo, se desarrolló un modelo matemático basado en el concepto de campo auto-consistente. Este algoritmo, bautizado en la correspondiente publicación como Auto-consistent Field Approximation Algorithm (AFA), proporciona una predicción del campo lumínico, incluyendo la evolución espectral del mismo a lo largo del camino óptico, para cultivos aclimatados a distintos valores de radiación. Dicha investigación se publicó en la revista Algal Research mediante el artículo titulado "Light distribution and spectral composition within cultures of micro-algae: Quantitative modelling of the light field in photobioreactors", en el que se valida el algoritmo con datos experimentales de dos cepas de estudio de la 
cianobacteria Synechocystis. Si bien los resultados fueron satisfactorios, el empleo de la ley de Lambert-Beer con un valor constante de atenuación no permite modelizar la parte del campo de luz con menor intensidad, donde el coeficiente de atenuación deja de ser constante y el comportamiento se desvía del exponencial. Por ello, se decidió modelizar el campo de luz con una función que generaliza el caso exponencial mediante el uso de cálculo fraccionario. Se empleó una función de Mittag-Leffler que cumplía con los requisitos formales y ofrecía un ajuste de los datos mejor al obtenido mediante la ley de LambertBeer. Como un hallazgo notable, se determinó que el valor de dicho parámetro, que caracteriza la función de Mittag-Leffler, era el mismo para los datos empíricos de las dos cepas estudiadas. Este trabajo se publicó en la contribución llamada "Estimation of the light field inside photosynthetic microorganism cultures through Mittag-Leffler functions at depleted light conditions" en la revista Journal of Quantitative Spectroscopy $\mathcal{E}$ Radiative Transfer. Después se procedió a utilizar sendos trabajos de investigación para calcular el campo de luz en un cultivo de Synechocystis y relacionarlo con su productividad máxima. En concreto se ha estudiado, como indicador del rendimiento de la fotosíntesis, la producción de oxígeno y los mecanismos respiratorios asociados a distintas intensidades de luz. Esta investigación está en su fase final y se está ultimando la escritura del artículo para enviarlo a una revista científica próximamente. Dicho manuscrito se titula "Experimental characterisation of Synechocystis sp. PCC 6803 cultures productivity up on light conditions". Finalmente, se está desarrollando una cuarta contribución titulada "Individual pigment contribution to overall in vivo absorption in Synechocystis sp. PCC 6803 cells". Esta investigación estudia la cantidad de luz absorbida por los cromóforos de Synechocystis en función del tipo de iluminación utilizada y calcula la concentración de pigmentos presentes en la célula. En resumen, la presenta tesis aporta herramientas de modelización al campo de la fotosíntesis con el objeto de avanzar en la integración de modelos matemáticos que relacionen aspectos ópticos con fenómenos biológicos y de esta manera apoyar iniciativas biotecnológicas en el campo del cultivo de cianobacterias. 


\section{Resum}

La present tesi doctoral, titulada "Contribution to the modelling of the light field distribution within Synechocystis sp. PCC 6803 cultures and its influence on cellular photosynthesis processes", engloba diversos treballs l'objectiu dels quals és avançar en la compressió de la distribució lumínica en cultius de cianobacteris i en els efectes de la llum sobre els mecanismes fotosintètics d'aquests microorganismes. Llavors, es tracta en definitiva d'un altre pas cap a la integració de models matemàtics sobre la fotosíntesi a nivell cel-lular i a escala de cultiu. En primer lloc, per a comprendre com es comporta un cultiu de bacteris fotosintètics, és fonamental predir la distribució del camp de llum al llarg del perfil del bioreactor, tant a nivell d'intensitat total, com pel que fa a la seua distribució de flux de fotons. La distribució de longituds d'ona present en el medi és important ja que molts processos de la fotosíntesi estan regulats per certes longituds d'ona i, per tant, estan modulats per la distribució espectral - el color - de la llum. En aquest sentit, si bé hi ha diverses metodologies que prediuen l'evolució espacial del camp de llum, aquestes aproximacions solen consistir en càlculs que aporten una solució no analítica i amb una càrrega computacional elevada. Per això, poder disposar d'una eina senzilla i a la vegada escalable amb altres models de processos fotosintètics, permetria avançar en la comprensió dels mateixos. Aprofitant les propietats inherents òptiques del cultiu, es va desenvolupar un model matemàtic basat en el concepte de camp auto-consistent. Aquest algoritme, batejat en la corresponent publicació com Auto-consistent Field Approximation Algorithm $(A F A)$, proporciona una predicció del camp lumínic, incloent l'evolució espectral del mateix al llarg del camí òptic, per a cultius aclimatats a diferents valors de radiació. Aquesta investigació es va publicar a la revista Algal Research mitjançant l'article titulat "Light distribution and espectral composition within cultures of micro-algae: Quantitative modelling of the light field in photobioreactors", en què es valida l'algoritme amb dades experimentals de dues soques d'estudi de la cianobacteri Synechocystis. 
Si bé els resultats van ser satisfactoris, l'ús de la llei de Lambert-Beer amb un valor constant d'atenuació no permet modelitzar la part del camp de llum amb menys intensitat, on el coeficient d'atenuació deixa de ser constant i el comportament es desvia del exponencial. Per això, es va decidir modelitzar el camp de llum amb una funció que generalitza el cas exponencial mitjançant l'ús de càlcul fraccionari. Es va emprar una funció de Mittag-Leffler que complia amb els requisits formals i oferia un ajust de les dades millor a l'obtingut mitjançant la llei de Lambert-Beer. Com una troballa notable, es va determinar que el valor d'aquest paràmetre, que caracteritza la funció de Mittag-Leffler, era el mateix per a les dades empíriques de les dues soques estudiades. Aquest treball es va publicar en la contribució anomenada "Estimation of the light field inside Photosynthetic microorganisme cultures through Mittag-Leffler functions at depleted light conditions" a la revista Journal of Quantitative Spectroscopy \& Radiative Transfer. Després, es va procedir a utilitzar sengles treballs d'investigació per calcular el camp de llum en un cultiu de Synechocystis i relacionar-lo amb la seua productivitat màxima. En concret s'ha estudiat, com a indicador del rendiment de la fotosíntesi, la producció d'oxigen i els mecanismes respiratoris associats a diferents intensitats de llum. Aquesta investigació està en la seua fase final i s'està ultimant l'escriptura de l'article per enviar-lo a una revista científica pròximament. Dit manuscrit es titula "Experimental characterisation of Synechocystis sp. PCC 6803 cultures productivity up on light conditions". Finalment, s'està desenvolupant una quarta contribució titulada "Individual pigment contribution to overall in vivo absorption in Synechocystis sp. PCC 6803 cells". Aquesta recerca estudia la quantitat de llum absorbida pels cromòfors de Synechocystis en funció del tipus d'il-luminació utilitzada i calcula la concentració de pigments presents en la cèl-lula. En resum, la present tesi aporta eines de modelització al camp de la fotosíntesis amb l'objecte d'avançar en la integració de models matemàtics que relacionen aspectes òptics amb fenòmens biològics i d'aquesta manera donar suport a iniciatives biotecnològiques en el camp del cultiu de cianobacteris. 


\section{Contents}

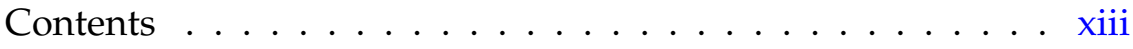

Main acronyms $\ldots \ldots \ldots \ldots \ldots$ xvii

Main symbols ................. . . . xxi

List of Figures . . . . . . . . . . . . . . . . . . . . . . . .

List of Tables . . . . . . . . . . . . . . . . . xxxiii

1 Introduction/Objectives 1

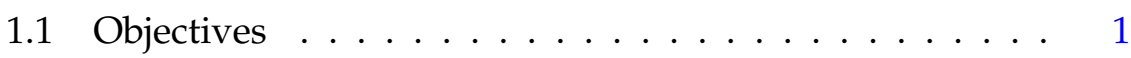

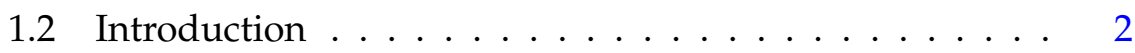

1.2.1 Synechocystis sp. PCC $6803 \ldots \ldots \ldots 2$

1.2.2 Light harvesting molecules .......... 3

1.2.3 Phycobilisome.............. 5

2 Light distribution and spectral composition within cultures of micro-algae $\quad 7$

2.1 Introduction $\ldots \ldots \ldots \ldots \ldots$

2.1.1 Light research in aquatic ecosystems . . . . . . . . . . 9

2.1.2 Modelling framework definition . . . . . . . . . . 16

2.2 Materials and Methods . . . . . . . . . . . . . . . 22

2.2.1 Validation strategy and modelling scheme implementation . . . . . . . . . . . 22

2.2.2 Linearity check of the $K_{d}$ vs. OD $_{750}$ relationship . 29

2.3 Results and discussion . . . . . . . . . . . . . 31

2.3.1 Optical spectra and analysis of the resulting IOPs 31

2.3.2 Attenuation profiles for white light exposure . . . 34

2.3.3 Attenuation profiles for colour light exposure . . 38

2.3.4 Spectrally dependent penetration depth and attenuation . . . . . . . . . . . . . 40 
2.3.5 Attenuation coefficient formula in Synechocystis

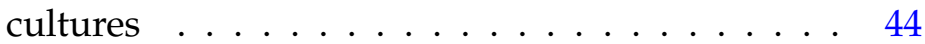

2.4 Conclusions and future work $\ldots \ldots \ldots$. . . . . . 46

2.5 Acknowledgements . . . . . . . . . . . . . . 47

3 Estimation of light field inside photosynthetic microorganism cultures through Mittag-Leffler functions 49

3.1 Introduction . . . . . . . . . . . . . . 50

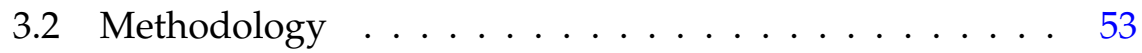

3.3 Fractional calculus in optics . . . . . . . . . . . 54

3.3.1 Data for modelling assessment . . . . . . . . . 55

3.4 Results . . . . . . . . . . . . . . . . 56

3.5 Conclusions . . . . . . . . . . . . . . . . . 59

3.6 Acknowledgements . . . . . . . . . . . . . . 60

4 Experimental characterisation of Synechocystis sp. PCC 6803 cultures productivity up on light conditions 61

4.1 Introduction . . . . . . . . . . . . . 63

4.1.1 Light within PBR cultures . . . . . . . . . . . 63

4.1.2 Biomass growth rates of Synechocystis cells . . . . 64

4.1.3 Oxygen related routes of Synechocystis cells . . . . 66

4.2 Materials and Methods . . . . . . . . . . . . . . 67

4.2.1 Strain and experimental design . . . . . . . . . 67

4.2.2 Optical measurements . . . . . . . . . . . . 67

4.2.3 Model description . . . . . . . . . . . . . . . . 69

4.3 Results and discussion . . . . . . . . . . . . . . 71

4.3.1 Main culture properties . . . . . . . . . . . 71

4.3.2 Modelled oxygen evolution . . . . . . . . . . 77

4.4 Conclusions . . . . . . . . . . . . . . . . 84

5 Individual pigment contribution to overall in vivo absorption in Synechocystis sp. PCC 6803 cells 95

5.1 Introduction . . . . . . . . . . . . . . . . 97

5.1.1 Light spectrum influence in photosynthesis . . . . 97

5.1.2 Absorption spectra of main pigments . . . . . . 98

5.1.3 Pigment content assessment . . . . . . . . . . 100 
5.2 Methodology . . . . . . . . . . . . . . . . . . . 101

5.2.1 In vitro absorption spectra of photosynthetic pigments . . . . . . . . . . . . . . . . . 101

5.2.2 Estimation of the package effect . . . . . . . . 102

5.2.3 Assessment of the cellular pigment concentration 105

5.2.4 Light absorption by each in vivo absorption spectra 106

5.3 Results and discussion . . . . . . . . . . . . . . 108

5.3.1 Package effect calculation . . . . . . . . . 108

5.3.2 Pigment quantification through the reconstruction of the in vivo absorption spectrum . . . . . . . 109

5.3.3 Absorption cross-section under different illumination environments . . . . . . . . . . . . . . . 114

5.4 Conclusions and future work . . . . . . . . . . . . 114

6 General discussion of the results

6.1 Article "Light distribution and spectral composition within cultures of micro-algae: Quantitative modelling of the light field in photobioreactors" . . . . . . . . . . . 117

6.2 Article "Estimation of the light field inside photosynthetic microorganism cultures through Mittag-Leffler functions at depleted light conditions" . . . . . . . . . . . . 120

6.3 Article "Experimental characterisation of Synechocystis sp. PCC 6803 cultures productivity up on light conditions" . 122

6.4 Article "Individual pigment contribution to overall in vivo absorption in Synechocystis sp. PCC 6803 cells" . . . . . . 125

7 Conclusions 



\section{Main acronyms}

AFA Auto-consistent Field Approximation Algorithm

AOP apparent optical property

APC allophycocyanin

att attenuance defined as the negative common logarithm of the transmittance

chl a chlorophyll a

CTD C-terminal domain

DCMU 3-(3,4-dichlorophenyl)-1,1-dimethylurea

DCQB 2,6-dichloro-1,4-benzoquinone

EET energy excitation transfer

ETC electron transport chain

FRAP fluorescence recovery after photobleaching

FRP fluorescence recovery protein

GME generalized master equation

GT glucose tolerant

hECN 3'-hydroxyechinenone

HL high light 
HPLC high-pressure liquid chromatography

IOP inherent optical property

L-D light-dark

LCM core membrane linker

LED light-emitting diode

LL low light

ML Mittag-Leffler

NPQ non-photochemical quenching

OCP orange carotenoid protein

OD optical density

ODE ordinary differential equation

opd absorbance defined as the negative common logarithm of the transmittance in the absence of scattering

PAR photosynthetically active radiation

PBR photobioreactor

PBS phycobilisome

PC phycocyanin

PCC Pasteur Culture Collection of Cyanobacteria

PFD photon flux density

PQ plastoquinone

PS photosystem

PSI photosystem I

PSII photosystem II

PSU photosynthetic unit 
RC reaction centre

ST state transitions

TE terminal emitter

UV ultraviolet

WT wild-type 



\section{Main symbols}

$\cos \varphi_{0} \quad$ Cosine of the angle of the photons to the vertical just below the water surface after refraction

$\rho_{\text {Chla }} \quad$ Chlorophyll a concentration in the culture $\left[\mathrm{mg} \mathrm{Chla} / \mathrm{m}^{3}\right.$ ]

$\mathrm{OD}_{\lambda} \quad$ Optical density at given wavelength $\lambda[\mathrm{nm}]$

$\Phi_{a}(\lambda) \quad$ Radiant flux, absorbed [W]

$\Phi_{b}(\lambda) \quad$ Radiant flux, scattered [W]

$\Phi_{c}(\lambda) \quad$ Radiant flux, attenuated [W]

$\Phi_{t}(\lambda) \quad$ Radiant flux, transmitted [W]

$\Phi_{i}(\lambda) \quad$ Radiant flux, incident [W]

$a(\lambda) \quad$ Absorption coefficient $\left[\mathrm{m}^{-1}\right]$

$b(\lambda) \quad$ Total scattering coefficient $\left[\mathrm{m}^{-1}\right]$

$c(\lambda) \quad$ Beam attenuation coefficient $\left[\mathrm{m}^{-1}\right]$

$j^{\star}\left(\lambda, E_{d, a c c}\right) \rho_{C h l a}$ specific coefficient at wavelength $\lambda$ and where culture is acclimated at intensity $E_{d, a c c} ; j$ is a placeholder that either stands for $a, b$ or $c\left[\mathrm{~m}^{2} \cdot \mathrm{mg} \mathrm{chl} \mathrm{a}^{-1}\right]$

$E_{0}(\lambda, z) \quad$ Scalar irradiance at the depth $\mathrm{z}$ and given a wavelength $\lambda$ $\left[\mu \mathrm{E} \cdot \mathrm{m}^{-2} \cdot \mathrm{s}^{-1}\right]$

$E_{d}(\lambda, z) \quad$ Downward irradiance at the depth $\mathrm{z}$ and at wavelength $\lambda$ $\left[\mu \mathrm{E} \cdot \mathrm{m}^{-2} \cdot \mathrm{s}^{-1}\right]$ 
$E_{d, P A R}(z)$ Downward irradiance integrated over PAR range and at the depth $\mathrm{z}\left[\mu \mathrm{E} \cdot \mathrm{m}^{-2} \cdot \mathrm{s}^{-1}\right]$

$\rho E_{d}(\lambda, z) \quad$ Spectral photon flux density of downward irradiance at the wavelength $\lambda$ and depth $\mathrm{z}\left[\mu \mathrm{E} \cdot \mathrm{m}^{-2} \cdot \mathrm{s}^{-1} \cdot \mathrm{nm}^{-1}\right]$

$E_{d, a c c} \quad$ Volume averaged and PAR integrated downward irradiance to which a given PBR culture has been acclimated $\left[\mu \mathrm{E} \cdot \mathrm{m}^{-2} \cdot \mathrm{s}^{-1}\right]$

$K_{0}(\lambda, z) \quad$ Attenuation coefficient for scalar irradiance at the depth $\mathrm{z}$ and at wavelength $\lambda\left[\mathrm{m}^{-1}\right]$

$K_{d}(\lambda, z) \quad$ Attenuation coefficient at the depth $\mathrm{z}$ and at wavelength $\lambda$ $\left[\mathrm{m}^{-1}\right]$

$\bar{K}_{d}\left(\lambda, E_{d, a c c}\right)$ Mean downward attenuation coefficient at wavelength $\lambda$ averaged for a culture acclimated at intensity $E_{d, a c c}\left[\mathrm{~m}^{-1}\right]$

$\bar{K}_{d, P A R}\left(E_{d, a c c}\right)$ Mean downward attenuation coefficient averaged within PAR range for a culture acclimated at intensity $E_{d, a c c}\left[\mathrm{~m}^{-1}\right]$

$\bar{K}_{d, P A R}^{\star}\left(E_{d, a c c}\right)$ Chlorophyll a-specific mean downward attenuation coefficient averaged within PAR range for a culture acclimated at intensity $E_{d, a c c}\left[\mathrm{~m}^{2} \cdot \mathrm{mg} \mathrm{chl} \mathrm{a}{ }^{-1}\right]$

I Exposure light intensity $\left[\mu \mathrm{E} \cdot \mathrm{m}^{-2} \cdot \mathrm{s}^{-1}\right]$

$I_{0} \quad$ Acclimation light intensity $\left[\mu \mathrm{E} \cdot \mathrm{m}^{-2} \cdot \mathrm{s}^{-1}\right]$

$x \quad$ Proportion of resting photosynthetic unit

$x^{*} \quad$ Proportion of activated photosynthetic unit

$x_{n f} \quad$ Proportion of non-functional photosynthetic unit

a Number of PSII units per cell

$Q_{P S I I} \quad$ Ratio of maximum to minimum PSII complexes

$Q_{P S I} \quad$ Ratio of maximum to minimum PSI complexes

$c_{P S I I} \quad$ Number of chl a molecules per PSII unit 
$c_{P S I} \quad$ Number of chl a molecules per PSI unit

c Chlorophyll content per cell $\left[\mathrm{fg} \mathrm{chla} \cdot \mathrm{cell}^{-1}\right]$

$c_{\max } \quad$ Maximal chlorophyll a mass per cell unit $\left[\mathrm{fg} \mathrm{chl} \mathrm{a} \cdot \operatorname{cell}^{-1}\right]$

$c_{\text {min }} \quad$ Minimal chlorophyll a mass per cell unit $\left[\mathrm{fg} \mathrm{chl} \mathrm{a} \cdot \mathrm{cell}^{-1}\right]$

$c_{\text {sol }} \quad$ Chlorophyll a content $\left[\mathrm{mg} \mathrm{chl} \mathrm{a} \cdot \mathrm{m}^{-3}\right]$

$\sigma \quad$ Chlorophyll-specific absorption-cross section [ $\mathrm{mg} \mathrm{chl} \mathrm{a} \cdot \mathrm{m}^{-3}$ ]

$P_{\text {gross }} \quad$ Gross photosynthesis rate $\left[\mathrm{mmol} \mathrm{O}_{2} \cdot \mathrm{mg} \mathrm{chl} \mathrm{a}^{-1} \cdot \mathrm{h}^{-1}\right]$

$P_{\text {net }} \quad$ Net photosynthesis rate $\left[\mu \mathrm{mol} \mathrm{O} \mathrm{O}_{2} \cdot \mathrm{mg} \mathrm{chl} \mathrm{a}^{-1} \cdot \mathrm{h}^{-1}\right]$

$P_{\max } \quad$ Maximum photosynthesis rate $\left[\mu \mathrm{mol} \mathrm{O} 2 \cdot \mathrm{mg} \mathrm{chl} \mathrm{a}^{-1} \cdot \mathrm{h}^{-1}\right]$

$R_{\text {dark }} \quad$ Dark respiration rate $\left[\mu \mathrm{mol} \mathrm{O}_{2} \cdot \mathrm{mg} \mathrm{chl} \mathrm{a}^{-1} \cdot \mathrm{h}^{-1}\right]$

$R_{\text {photo }} \quad$ Photo-respiration rate $\left[\mu \mathrm{mol} \mathrm{O}_{2} \cdot \mathrm{mg} \mathrm{chl} \mathrm{a}^{-1} \cdot \mathrm{h}^{-1}\right]$

$Q_{a}^{*} \quad$ Package effect factor

$a_{\text {sol }} \quad$ Absorption coefficient for an ideally dispersed pigment solution $\left[\mathrm{m}^{-1}\right]$

$\rho^{\prime} \quad$ Dimensionless factor ruling the discreteness of absorption

$c_{i} \quad$ Intracellular chlorophyll concentration

$d_{i} \quad$ Cell internal diameter

$F(d) \quad$ Cell size distribution function

$n^{\prime} \quad$ Imaginary part of the refractive index 



\section{List of Figures}

2.1 Summarised modelling scheme to obtain in silico photosynthetically active radiation (PAR) attenuation profiles and spectral ones. . . . . . . . . . . . .

2.2 Emission spectra in terms of relative power of the LED lamps used for the attenuation trials. Graph colours represent each LED characteristic colour (blue, green and red), whereas black curve corresponds to the white LED.

2.3 Relationship between chlorophyll a and $\mathrm{OD}_{750}$ value of both strains (wild-type (WT) in green colour and Olive in brown one) grown at incident $100 \mu \mathrm{mol}$ photons $\cdot \mathrm{m}^{-2}$. $s^{-1}$. Dots represents experimental data and the line represents the lineal regression. . . . . . . . . . . . . 30

2.4 (A) Absorption $a^{\star}\left(\lambda, E_{d, i}\right)$ and (B) total scattering $b^{\star}\left(\lambda, E_{d, i}\right)$ chlorophyll a (chl a)-specific coefficients within PAR waveband of wild-type and Olive strain (green and brown colour, respectively) grown at incident light intensities of 40, 100 (dashed) and 170 (dotted) $\mu \mathrm{mol}$ photons $\cdot \mathrm{m}^{-2} \cdot \mathrm{s}^{-1}$ in a 4-cm flat-type photobioreactor. . . . . . . . . . . .

2.5 Modelled photon absorption contribution of wild-type and Olive strain (green and brown colour, respectively) grown at average light intensities of 20, 60 (dashed) and 100 (dotted) $\mu$ mol photons $\cdot m^{-2} \cdot s^{-1}$ for each wavelength within PAR range following Kirk's formula. . . . . . . . . 
2.6 Modelled chlorophyll-specific downward attenuation function $\bar{K}_{d}^{\star}\left(\lambda, E_{d, i}\right)$ for wild-type and Olive strain (green and brown colour, respectively) at the incident irradiance values of 40, 100 (dashed) and 170 (dotted) $\mu$ mol photons . $m^{-2} \cdot s^{-1} \ldots \ldots \ldots \ldots \ldots \ldots \ldots$

2.7 Left panel: Light attenuation profiles of Synechocystis WT (A) and Olive (B) strain cultures exposed to 2,000 $\mu \mathrm{mol}$ photons. $m^{-2} \cdot s^{-1}$ of white LED light at five different $\mathrm{OD}_{750}$ concentrations (0.1, 0.5, 1.0, 2.5 and 5.0) are depicted. Dots are the original source samples Lea-Smith et al. (2014) and lines the simulation outcome. Darker colours correspond to denser suspensions. Right panel: Light attenuation profiles of Synechocystis WT (C) and Olive (D) strain cultures exposed to $1,000 \mu \mathrm{mol}$ photons $\cdot \mathrm{m}^{-2} \cdot \mathrm{s}^{-1}$ of four different LED lamps at an $\mathrm{OD}_{750}$ concentration of 1.0 are shown. Dots are the original source samples. Graph colours represent each LED characteristic colour (blue, green and red), whereas black curve corresponds to the white LED. . . . . . . . . . . . . . . . . . 36

2.8 Chlorophyll a-specific mean downward attenuation coefficient $\bar{K}_{d, \text { PAR }}^{\star}\left(E_{d, a c c}\right)$ comparison for WT and Olive strains (green and brown colour, respectively) between experimental (dots) and modelled values at the given densities. Experimental coefficients were obtained from the white lamp exposure assays at 2,000 $\mu \mathrm{mol}$ photons $\cdot \mathrm{m}^{-2} \cdot \mathrm{s}^{-1}$ and calculated as irradiance-weighted attenuation coefficients, whereas the in silico values were directly obtained by dividing by the chlorophyll amount for each optical density . . . . . . . . . . . . . . 4 40 
2.9 Penetration depth at which irradiance drops to $10 \%$ of the initial value at each wavelength within PAR range for WT and Olive strains (green and brown colour, respectively). Simulation conditions correspond to Synechocystis WT cultures grown at typical photobioreactor (PBR) densities, i.e. $1.0 \mathrm{OD}_{750}$, and acclimatised to a lamp irradiance of $80 \mu \mathrm{mol}$ photons $\cdot \mathrm{m}^{-2} \cdot \mathrm{s}^{-1}$ of white light. Blue dots correspond to Spirulina platensis depths estimated from experimental attenuation coefficients. . . . . . . .

2.10 Modelled spectral photon flux densities $\rho E_{d}(\lambda, z)$ within simulated Synechocystis WT strain cultures are depicted, where cells are adapted to white light of $80 \mu \mathrm{mol}$ photons. $m^{-2} \cdot s^{-1}$ but momentarily exposed to $1,000 \mu$ mol photons. $m^{-2} \cdot s^{-1}$ of different colour LED lamps (white, blue, green or red light) at an $\mathrm{OD}_{750}$ concentration of 1.0. Remaining photon flux densities at 0, 1 (dashed) and $2 \mathrm{~cm}$ (dotted) are shown. Inset plot shows whole graphs with the same units in both axes. . . . . . . . . . . . . .

2.11 Modelled mean downward attenuation coefficient within PAR range $\bar{K}_{d, \mathrm{PAR}}\left(E_{d, a c c}\right)$ for WT cultures exposed to lamp intensities of 20 and $100 \mu \mathrm{mol}$ photons $\cdot \mathrm{m}^{-2} \cdot \mathrm{s}^{-1}$ in a 4-cm depth PBR at constant cell-densities up to 25,000 $\mathrm{mg} \mathrm{chl} \mathrm{a} \cdot \mathrm{m}^{-3}$ are depicted (right vertical axis). Additionally, resulting average irradiance $E_{d, a c c}$ is also plotted for such suspensions (dotted, left vertical axis). Green and blue curves stand for WT cultures grown at 20 and $100 \mu \mathrm{mol}$ photons $\cdot \mathrm{m}^{-2} \cdot \mathrm{s}^{-1}$ incident radiation and similarly red and yellow curves represent Olive cultures cultivated at 20 and $100 \mu \mathrm{mol}$ photons $\cdot \mathrm{m}^{-2} \cdot \mathrm{s}^{-1}$ incident radiation. . . . . . . . . . . . . . . . 
3.1 Light attenuation profiles of Synechocystis WT strain cultures exposed to $2000 \mu \mathrm{mol}$ photons $\cdot \mathrm{m}^{-2} \cdot \mathrm{s}^{-1}$ of white light-emitting diode (LED) light at five different $\mathrm{OD}_{750}$ concentrations (0.1, 0.5, 1.0, 2.5 and 5.0) are depicted. Dots are the original source samples and lines the modelling outcome (dashed lines Lambert-Beer law, solid lines Mittag-Leffler function). Darker colours correspond to denser suspensions. . . . . . . . . . . . . . . 57

3.2 Light attenuation profiles of Synechocystis Olive strain cultures exposed to $2000 \mu \mathrm{mol}$ photons $\cdot \mathrm{m}^{-2} \cdot \mathrm{s}^{-1}$ of white LED light at five different $\mathrm{OD}_{750}$ concentrations $(0.1,0.5$, 1.0, 2.5 and 5.0) are depicted. Dots are the original source samples and lines the modelling outcome (dashed lines Lambert-Beer law, solid lines Mittag-Leffler function). Darker colours correspond to denser suspensions. . . . . . . 58

4.1 Culture specific growth and $\mathrm{CO}_{2}$ uptake under continuous red light. $(\bigcirc)$ markers correspond to the specific growth rate and $(\triangle)$ to carbon uptake. Error bars indicate the standard deviation of the replicates. . . . . . . 72

4.2 Chlorophyll a and carotenoid content under continuous light. $(\bigcirc)$ markers correspond to the the chlorophyll aamount $(\triangle)$ to carotenoidal concentration. Error bars indicate the standard deviation of the replicates. . . . . . . . . 73

4.3 Measured $\mathrm{O}_{2}$ evolution at different mean acclimation and exposure light intensities in $\mu \mathrm{mol}$ photons $\cdot \mathrm{m}^{-2} \cdot \mathrm{s}^{-1}$ units: $18(-), 29(-), 49(-), 92(-), 171(-), 373(-)$ and $464(-)$. Experimental mean values are displayed as dots with error bars, while the corresponding piecewise cubic hermite interpolating polynomial is drawn as a solid curve. . . . . . . . . . . . . . . . . 74 
4.4 Measured dark respiration $\mathrm{O}_{2}$ evolution at different mean acclimation and exposure light intensities in $\mu$ mol photons. $m^{-2} \cdot s^{-1}$ units: $18(-), 29(-), 49(-), 92(-), 171$ $(-), 373$ (-) and $464(-)$. Experimental mean values are displayed as dots with error bars, while the corresponding simple linear regression line is drawn as a solid line.

4.5 Chlorophyll a mass per cell under continuous light. Exponential fit (-) and through a mass balance estimated minimal chlorophyll at saturation intensity (---) are also shown. . . . . . . . . . . . . .

4.6 Specific growth under different optical density values, light intensity combinations and frequency values. Star markers $(*)$ represent cultures under 220 mol photons . $m^{-2} \cdot s^{-1}$ continuous red light at $0.1,0.2$ and $0.4 \mathrm{OD}_{720}$ (the lower density, the higher the growth) and for the sake of convenience, they are plotted under the zero duration period. Circles display $(\bigcirc)$ cultures at $0.1 \mathrm{OD}_{720}$ with dissimilar switch frequencies with a red light combination of LL/HL 40/400 $\mu$ mol photons $\cdot m^{-2} \cdot s^{-1}$. The other symbols represent duplicated cultures of $0.1 \mathrm{OD}_{720}$ with an alternation frequency of $16 \mathrm{~s}$, whereas upwardpointing triangles $(\triangle)$, squares $(\square)$ and downward-pointing triangles $(\nabla)$ depict the following combination of $\mathrm{LL} / \mathrm{HL}$ intensities: $110 / 330,77 / 363,0 / 440 \mu$ mol photons $\cdot m^{-2}$. $s^{-1}$. Lowest doubling times indicated. . . . . . . . . .

4.7 Steady state oxygen evolution $\left(\mu \mathrm{mol} \mathrm{O}_{2} \cdot \mathrm{mg} \mathrm{chl} \mathrm{a}^{-1} \cdot \mathrm{h}^{-1}\right)$ with respect to average irradiance. Dots correspond to experimental value, while lines depict the modelled outcome: gross photosynthetic $\mathrm{O}_{2}$ rates $P_{\text {gross }}(-)$, related net photosynthesis performance $P_{\text {net }}(---)$, dark respiration $R_{\text {dark }}(-\cdot \cdot)$ and photo-respiration $R_{\text {photo }}(\cdots \cdots)$. . . . . 80

4.8 Proportion of absorbed light transformed into $\mathrm{O}_{2}$ molecules, net $(-)$ and gross $(--)$ productivity. . . . . . . . . 
4.9 Modelled $\mathrm{O}_{2}$ generation $\left(\mu \mathrm{mol} \mathrm{O}_{2} \cdot \mathrm{L}^{-1} \cdot \mathrm{h}^{-1}\right)$ with respect to the culture chlorophyll a concentration $\left(\mathrm{mg} \cdot \mathrm{m}^{-3}\right)$ at different growth light intensities. Simulations of cultures acclimatised to the following incident intensities in $\mu$ mol photons. $m^{-2} \cdot s^{-1}$ are plotted: light intensities in $\mu$ mol photons . $m^{-2} \cdot s^{-1}$ units: $100(-), 200(-), 400(-), 600(-)$, $800(-) . \mathrm{O}_{2}$ volumetric production results from a single average acclimation intensity, but cells situated at then different equidistant positions along the optical path. . .

4.10 Downward coefficients $K_{d}$ in $\mathrm{m}^{-1}$ at different average growth intensities gathered via IOPs calculation from experimental absorption and scattering spectra at the given intensities: light intensities in $\mu \mathrm{mol}$ photons $\cdot \mathrm{m}^{-2} \cdot \mathrm{s}^{-1}$ units: $18(-), 29(-), 49(-), 92(-), 171(-), 373(-)$ and $464(-) . \quad \ldots \ldots \ldots$. . . . . . . . . . . . 89

4.11 Remaining light intensity leaving the PBR at the centre of the reactor surface $(z=2.4 \mathrm{~cm})$ with respect to the incident one for cultures grown at different red light intensities supplemented with $25 \mu \mathrm{mol}$ photons $\cdot \mathrm{m}^{-2} \cdot \mathrm{s}^{-1}$ of blue radiation. The colour represents the momentarily exposure intensity: blue one indicates momentary pure blue exposure ( $25 \mu \mathrm{mol}$ photons $\left.\cdot \mathrm{m}^{-2} \cdot \mathrm{s}^{-1}\right)$, red one implies only red exposure without addition of blue photons and purple colour corresponds to the original blue-red growth light. $(\bigcirc)$ markers represent measured data and $(\times)$ ones correspond to modelled values. . . . . . . . . .

4.12 The cellular absorption cross-section $\xi$ relationship to cellular chlorophyll content per cell $c$. Linear fit equation and corresponding coefficient of determination $\mathrm{R}^{2}$ are displayed in the figure, being the gathered parameters of Equation (4.5), $\xi_{\min }=9.1 \cdot 10^{4} \mathrm{~m}^{2} \cdot \mathrm{mg} \mathrm{chl} \mathrm{a}^{-2} \cdot$ cell and $m_{\xi}=0.0068 \mathrm{~m}^{2} \cdot \mathrm{mg} \mathrm{chl} \mathrm{a}^{-1} \ldots \ldots \ldots \ldots$. . . . 
4.13 Relationship between dark respiration and photosynthetic yield for different light intensities. Linear fit equation and corresponding coefficient of determination $\mathrm{R}^{2}$ are displayed in the figure, being the gathered parameters of Equation (4.14), $m=2.4 \mu \mathrm{mol} \mathrm{O} 2 \cdot \mathrm{mg} \mathrm{chl} \mathrm{a}^{-1} \cdot \mathrm{h}^{-1}$ and slope $\gamma=0.13$ for the studied light interval. . . . . . . . .

4.14 Scheme of the PBR framework to scale. LED surface is shown following a colourmap that indicates the irradiance entering the culture at different points relative to the maximum value. PBR area is depicted with green colour. Dimensions are given in millimetres. . . . . . . .

5.1 Photon flux spectra of common light sources for photosynthetic microorganisms cultures along the PAR range indicated in relative units. Photon flux distribution of each light source is depicted with the colours that belong to the corresponding wavelength. . . . . . . . . . . .

5.2 Computed package effect factor $\mathrm{Q}_{\mathrm{a}}^{*}$ for Synechocystis WT along the PAR range. . . . . . . . . . . . . . . . . 108

5.3 Reconstructed Synechocystis absorption spectrum along the PAR range of cells acclimatised to a total intensity of $100 \mu \mathrm{mol}$ photons $\cdot \mathrm{m}^{-2} \cdot \mathrm{s}^{-1}$ of cool white LED light. Black signature (-) corresponds to in vivo spectrum, while grey curve (---) is the reconstructed absorption. Computed in vivo absorption contribution of each pigment is also depicted: chlorophyll a (-), carotenoids (-) [ordered with respect to their maximum absorption peak, $\beta$-carotene $(-)$, myxoxanthophyll $(-\cdots)$, zeaxanthin (--), echinenone ( $\cdots)$ and $3^{\prime}$ - hydroxyechinenone $(\cdots)$ ], allophycocyanin $(-)$ and phycocyanin $(-)$. 3' hydroxyechinenone spectrum can be distinguished from echinenone's one since the former one is smaller. . . . . . 110 
5.4 Phycobilisomes absorptive spectral characteristics of Synechocystis cells acclimatised to a total intensity of $100 \mu \mathrm{mol}$ photons $\cdot \mathrm{m}^{-2} \cdot \mathrm{s}^{-1}$ of cool white LED light. PBS absorption contribution with respect to the cellular one (-) and total PBS absorption coefficients (-) expressed in $\mathrm{m}^{-1}$ units are also depicted. Dashed vertical lines approximately indicate the wavelength range $(550-650 \mathrm{~nm})$ in which PBS absorption rules over chlorophyll a one. . . . . . . . . . . . 113

5.5 Absorption cross-section of individual pigments for Synechocystis cells acclimatised at $\mathrm{X}$ intensity under different illumination set-ups. Pigment absorption is partitioned in the main light harvesting structures present in the cells (including their chromophores): PBS rod (PC - ), PBS core (APC $\square$ ) and PBS TE (APC $\square$ ), PSII (Chl. a $\square$ ), PSI (Chl. a -$)$, light harvesting carotenoid of PSII $(\beta$ car. $\square)$, and the one for PSI: ( $\beta$-car. $\square)$. The employed light sources with their corresponding emission spectra are the ones shown in Figure 5.1. These sources can be divided into solar light (S-sun), white light lamps with a broad spectrum (I-incandescent bulb, F-fluorescent lamp, H-halogen lamp), C-cool white LED and W-warm white LED) and monochromatic LEDs. In the last case, they present a Gaussian shape, whose peak is located for each lamp at the following wavelengths in $\mathrm{nm}$, including the colour of the emission peak wavelength): B-blue 438, Tturquoise 480, G-green 544, A-amber 590, O-orange 624, R-red $674 . \ldots \ldots \ldots$. . . . . . . . . . . 116 


\section{List of Tables}

2.1 List of symbols and abbreviations. . . . . . . . . . . 1

5.1 Main characteristics and inferred concentrations of the pigments used for the absorption spectrum reconstruction. References included in the absorption coefficient column imply a full knowledge of the spectrum, including its numeric magnitude. Alternatively, for the references shown next to the absorption peak, the corresponding coefficient are indirectly hypothesised as explain in the text. The measured chlorophyll a concentration value was $1,650 \mathrm{mg} \mathrm{chl} \mathrm{a} \cdot \mathrm{m}^{-3}$.

* The reference for the phycobilin content comparison is Tsunoyama et al. (2009), whereas for the carotenoids is the mean of the following works Takaichi et al. (2001); Touloupakis et al. (2015); Kłodawska et al. (2014); Vajravel et al. (2016); Zakar et al. (2017). ${ }^{* *} 3^{\prime}$-Hydroxyechinone content was imposed to match literature one. ${ }^{a}$ Faccio et al. (2014), ${ }^{b}$ MacColl (2004), ${ }^{c}$ Chábera et al. (2011), ${ }^{d}$ Harmut and Lichtenthaler (1987), ${ }^{e}$ Hiyama et al. (1969), ${ }^{f}$ Hertzberg et al. (1971), ${ }^{g}$ Aasen and Jensen (1966), ${ }^{h}$ Warren and Weedon (1958), ${ }^{i}$ Tsunoyama et al. (2009), ${ }^{j}$ Takaichi et al. (2001).112 



\section{1}

\section{Introduction/Objectives}

\subsection{Objectives}

The objective of the present thesis is to delve deeper in the understanding of how light is distributed in a microorganism photosynthetic culture, in the impact thereof on photosynthesis performance and in the contribution of each pigment to cell absorption up on the illumination environment. The studied microorganism is Synechocystissp. PCC 6803 (hereafter simply referred to as Synechocystis), one of the most promising biotechnological platforms for industrial applications due to its genetic convertibility and suitable properties for mass cultivation.

It is worth mentioning that this thesis contributes to this field by bringing into play different methodologies that combine pure optical aspects with biological ones, representing another step towards integrative optical-photosynthetic modelling. In this regard, the here presented research generalises light treatment to account with its spectral properties and from the culture to the cell.

The particular objectives of this research can be split into the following items: 
- Estimate the light field distribution within Synechocystis WT and Olive mutant cultures at different cell-densities and acclimation intensities by means of an exponential function approach

- Improve the corresponding simulation of light attenuation within suspensions of both strains through the application of fractional calculus, namely leveraging the properties of the Mittag-Leffler function

- Study the main characteristics of Synechocystis cultures up on different irradiance values

- Assess the photosynthetic productivity of Synechocystis cells in terms of $\mathrm{O}_{2}$ evolution up on different irradiance values and for different cell-densities

- Reconstruct the in vivo absorption spectra of Synechocystis strains from in vitro signatures of the cell pigments and by doing so, the concentration of the main pigments can be calculated

- Evaluate the contribution of each pigment to overall light absorption depending on the type of light source employed

\subsection{Introduction}

\subsubsection{Synechocystis sp. PCC 6803}

Synechocystis sp. PCC 6803 is a unicellular non-nitrogen fixing coccoid cyanobacterium, and the first phototrophic organism whose genome was fully sequenced Kaneko et al. (1996), being also the fourth genomesequenced organism. Synechocystis is one of the most popular organisms for genetic, physiological and bioengineering studies due to two main factors: it is naturally transformable by exogenous DNA Grigorieva and Shestakov (1982), making silencing or adding genes from other organisms easier, and it grows heterotrophically with glucose Rippka et al. (1979), allowing a wider set of experimental conditions. 
From the total of 3167 genes, 128 are involved in the various stages of the photosynthesis, and 224 are significantly homologous to the genes of higher plant plastids, including those related to PSI and PSII, the electron transfer chain, $\mathrm{CO}_{2}$ fixation... Ikeuchi and Tabata (2001).

Synechocystis sp. PCC 6803 owns several characteristics that make it suitable for the production of alternative fuels too Quintana et al. (2011):

- The cells contain a high quantity of lipids in their thylakoid membrane.

- Its photosynthetic rate is higher than algae and higher plants performance per biomass unit.

- It requires low basic nutritional requirements: gaseous $\mathrm{CO}_{2}$, mineral salts, water and light as energy source.

- Cultivation is relatively simple and cheap and moreover it can resist mid-high temperatures Červenỳ et al. (2015) and basic pH environments Buck and Smith (1995).

Due to its genetic versatility and metabolic features, Synechocystis has been used to produce a wide range of fuels: ethanol Dexter and $\mathrm{Fu}$ (2009) or biodiesel Rittmann (2008), among others.

\subsubsection{Light harvesting molecules}

Photosynthetic organisms have photo-active molecules, also referred to as chromophores, which can capture radiation, thus getting energetically excited, and being able to transmit the generated excitons to the surrounding chromophore molecules. Such excitation transfer chains end up at the reaction centre (RC) of a photosystem, where excitation serves to generate the electron flow for photosynthesis Broess et al. (2006).

In the particular case of our strain of study, Synechocystis cells possess three main groups of photo-active pigments: chlorophylls, phycobiliproteins MacColl (1998) and carotenoids Zakar et al. (2016). The 
most abundant chlorophyll in this cyanobacterium is the type a $\mathrm{Mu}-$ rakami and Fujita (1988), whereas there are two main phycobiliproteins forming the phycobilisome (PBS) antennae: the phycocyanin (PC) conforming the rods and the allophycocyanin (APC) building the core of the antennae Arteni et al. (2009). Regarding the carotenoids, five main molecules can be found: the carotene $\beta$-carotene and the xanthophylls zeaxanthin, myxoxanthophyll, echinenone and 3' - hydroxyechinenone Takaichi et al. (2001).

With respect to the absorption signature of Synechocystis, blue and farred light is captured by chlorophyll a, whereas carotenoids mainly harvest blue-green radiation. Alternatively, yellow and orange irradiance is primarily taken up by phycobilins Allen and Mullineaux (2004). In fact, PBS absorbs light in the wavelength range from 550 to $680 \mathrm{~nm}$, since PC absorption peak roughly lies at $620 \mathrm{~nm}$ and APC at $660 \mathrm{~nm}$ in vivo conditions Simis and Kauko (2012).

Regarding the number of molecules conforming the cells, dissimilar concentration values for all pigments present in Synechocystis are reported in literature. Nevertheless, the latter is not surprising as most of the pigments are highly dependent on the growth dynamics and light conditions at which the cells are exposed to. For example, chlorophyll concentration follows an inversely proportional relationship with respect to the acclimation irradiance Dubinsky and Stambler (2009) as phycobiliproteins content does Kana and Glibert (1987). In this way, cells are able to regulate their absorption capacity and so optimise photosynthesis and avoid photoinhibition Murata et al. (2007). Such dynamic behaviour has to be taken into account when long-term photoacclimation mechanisms are to be modelled. This is due to the fact that variations in the pigment concentration lead to a modified light input through all pigments present in the cell and so, photosynthetic electron chain can be affected too. 


\subsubsection{Phycobilisome}

Amongst all light harvesting compounds in cyanobacteria, there are two that stand out among the rest: both phycobilinproteins conforming the phycobilisomes (PBS). The PBS structures are peripheral membrane complexes anchored to the thylakoid membrane by the outer surface and account for half of the soluble proteins fraction Ughy and Ajlani (2004). They act in the initial step of photosynthesis, harvesting the light, since their basic building blocks are chromophorylated phycobiliproteins. Phycobilisomes' structure consists of six peripheral rods that radiate from an inner core Elmorjani et al. (1986). In Synechocystis sp. PCC 6803, the chromophore present in the rods is C-phycocyanin (PC) and each rod has three disc-shaped hexamers (12 bilins per hexamer) Arteni et al. (2009). Furthermore, the core is formed by three cylinders of allophycocyanin (APC) molecules and there are exactly four APC trimers per cylinder. Furthermore, the two basal cylinders contain two $\mathrm{APC}_{660}$, two $\mathrm{APC}_{680}$ and $\mathrm{APC}$-like subunits. The $\mathrm{APC}_{680}$ trimer, also named as terminal emitter (TE) Capuano et al. (1991), transfers the energy to the photosystems PSI and PSII, specifically to the chlorophyll antenna and reaction centres Mullineaux (1992); Rakhimberdieva et al. (2001). The TE is a fundamental unit within APC core as it helps in fine-tuning the energy transfer towards PSI and PSII complexes through $\mathrm{ApcD}$ and $\mathrm{ApcE}$ subunits respectively McConnell et al. (2002), but its main role is the electrostatic interaction with the thylakoid membrane, the PSII and the PSI surfaces Adir (2005); David et al. (2011).

Phycobilisomes can handle optimal light absorption under low irradiance conditions. But under high light stress, they are involved in the protection against photoinhibition. Additionally, it is known that PBS interaction with both photosystems also depends on steric and spatial characteristics of the photosystems. Indeed, PSII dimers present a flat surface without extra subunits on the cytoplasmic side Bald et al. (1996); Ferreira et al. (2004), allowing the PBS to couple directly over its top and transfer excitation to PSII through an $\mathrm{APC}_{680}$ trimer Mullineaux 
(2008). By contrast, PSI complex has several subunits with some domains on the cytoplasmic side Jordan et al. (2001), resulting in a rigid structure of difficult and partial access, precluding a tight union of PBS to PSI complexes Rakhimberdieva et al. (2001). This directly quantitatively affects the capacity to divert excitation energy towards PSI complexes with respect to PSII units. 


\section{2}

\section{Light distribution and spectral composition within cultures of micro-algae}

Title: "Light distribution and spectral composition within cultures of micro-algae: Quantitative modelling of the light field in photobioreactors"

Reference: Algal Research 23 (2017) 166-177

Authors: David Fuente ${ }^{a, *}$, Joseph Keller ${ }^{b}$, J. Alberto Conejero ${ }^{c}$, Matthias Rögner $^{b}$, Sascha Rexroth ${ }^{b}$, Javier F. Urchueguía ${ }^{a}$

${ }^{a}$ Instituto de Aplicaciones de las Tecnologías de la Información y de las Comunicaciones Avanzadas, Universitat Politècnica de València, València, Spain

${ }^{b}$ Plant Biochemistry, Faculty of Biology and Biotechnology, Ruhr University Bochum, Germany

${ }^{c}$ Instituto Universitario de Matemática Pura y Aplicada, Universitat Politècnica de València, Valencia, Spain

* Corresponding author: dafueher@upv.es 


\section{Abstract}

Light, being the fundamental energy source to sustain life on Earth, is the external factor with the strongest impact on photosynthetic microorganisms. Moreover, when considering biotechnological applications such as the production of energy carriers and commodities in photobioreactors, light supply within the reactor volume is one of the main limiting factors for an efficient system. Thus, the prediction of light availability and its spectral distribution is of fundamental importance for the productivity of photo-biological processes.

The light field model here presented is able to predict the intensity and spectral distribution of light throughout the reactor volume based on the incident light and the spectral characteristics of the photosynthetic microorganism. It takes into account the scattering and absorption behaviour of the micro-algae, as well the adaptation of the biological system to different light intensities.

Although in the form exposed here the model is optimized for photosynthetic microorganism cultures inside flat-type photobioreactors, the theoretical framework is easily extensible to other geometries. Our calculation scheme has been applied to model the light field inside Synechocystis sp. Pasteur Culture Collection of Cyanobacteria (PCC) 6803 wild-type (WT) and Olive antenna mutant cultures at different cell-density concentrations exposed to white, blue, green and red LED lamps, delivering results with reasonable accuracy, despite the data uncertainties. To achieve this, Synechocystis experimental attenuation profiles for different light sources were estimated by means of the BeerLambert law, whereby the corresponding downward irradiance attenuation coefficients $K_{d}(\lambda)$ were obtained through inherent optical properties of each organism at any wavelength within the photosynthetically active radiation band. The input data for the algorithm are chlorophyllspecific absorption and scattering spectra at different mean acclimatisation irradiance values for a given organism, the depth of the pho- 
tobioreactor, the cell-density and also the intensity and emission spectrum of the light source.

In summary, the model is a general tool to predict light availability inside photosynthetic microorganism cultures and to optimize light supply, in respect to both intensity and spectral distribution, in technological applications. This knowledge is crucial for industrial-scale optimisation of light distribution within photobioreactors and is also a fundamental parameter for unravelling the nature of many photosynthetic processes.

Keywords: absorption, scattering, attenuation, inherent optical properties, modelling, Synechocystis

\subsection{Introduction}

\subsubsection{Light research in aquatic ecosystems}

\section{Introduction to optics in biology}

Photosynthesis is a very active research field in the life sciences due to the crucial importance of photosynthetic organisms as the fundamental source of all biomass in our planet. Particularly, much research has been done in understanding how light behaves inside different water bodies, such as inland, coastal and oceanic ecosystems.

Concurrently, bio-optical researchers have developed several methodologies to estimate optical properties. In the year 1961 Preisendorfer defined the concept of inherent optical property (IOP) and apparent optical property (AOP) of water bodies, founding optical oceanography Preisendorfer (1961). Relating IOPs with AOPs has been an ongoing effort since then, and authors have studied, experimentally as well as theoretically Antoine et al. (2014), the optical characteristics of water and cell suspensions as a function of water body features and 
metabolic variables such as the energy stored by algae upon light conditions Morel (1991).

But oceanic optics is not the only field of interest in the study of light interaction with microorganisms. During the last 30 years, more interest has progressively been devoted to the development of closed photobioreactors (PBRs), aimed at the production of many substances of interest ranging from nutra- and pharmaceuticals, to bioenergetic compounds Touloupakis et al. (2016b), Chisti (2007). As dense cultures are preferred to maximise production, light is normally the limiting factor to obtain a cost effective PBR operation. Although dense suspensions are a priori more appropriate for an efficient PBR utilisation Richmond (2003), too concentrated cultures may increase operating costs Norsker et al. (2011) and completely deplete the system of light in most the external layers Gitelson et al. (1996) as well. Therefore, optimisation of illumination conditions and cell density is required for improving overall photosynthesis performance and to minimise dark respiration and thus for achieving an optimal design of large-scale photobioreactors Posten (2009).

From the point of view of light propagation, there are important differences between the conditions in open waters or inside a PBR aqueous phase. The use of artificial light sources in many PBR set-ups, unnatural light cycles, the geometry of the arrangement itself and its inherent limitation in culture depth, not present in most open waters, are just some of the differentiating factors. A crucial topic is the question of stratification. Whilst in open waters a given equilibrium stratification is established within the photic zone and substantial differences may be found in microorganism concentration and composition depending on depth, inside a PBR efforts are usually oriented towards obtaining a good mixing so that the photosynthetic cells can rapidly move towards the external and internal zones of the reactor. Accordingly, the culture inside the PBR volume is usually regarded as being homogeneous.

Regarding the strategies to describe light distribution within water bodies, authors have either used algorithms that calculate the light field 
based on the radiative transfer equation describing light-matter interaction Ahmad and Fraser (1982) or have applied stochastic methods such as Monte Carlo simulations Kirk (1981); Dauchet et al. (2013), which allow researchers to statistically follow the fate of individual photons within the medium. Relevant works based on this strategy have been published in the last decades. In this regard, in some cases the light field prediction is linked with experimental cell growth Niizawa et al. (2014); Blanken et al. (2016) or coupled biomass production is modelled following a classical growth law such as Monod-type Cornet et al. (1992). Several applications on different reactor shapes such as torus photobioreactors Pottier et al. (2005) or open ponds Heinrich et al. (2012) can be found.

In our approach we aim at creating a procedure in between the simple light models and exceedingly detailed simulations in order to get a holistic view of the interaction of light and biomass based on the IOPs of the cells of interest, which has not been described in literature and is novel to the field. To do so, we will derive a relationship connecting the light field profile within a PBR suspension knowing the cell density, lamp emission spectrum, culture depth, absorption and scattering coefficients of the culture acclimatised to different light intensities. Making some simplifying assumptions we arrive at an expression that can be easily solved and can even give rise to an analytic relationship between operating parameters of the culture and includes in an implicit manner photo-adaptation of the cells. Furthermore, we have tested our scheme using information from two sources, completed with our own experiments, on two different strains of Synechocystis sp. PCC 6803 (hereafter referred to as Synechocystis), the wild-type and the Olive mutant. The latter is a strain with truncated phycobilisome structure, where the phycobilisome core is present but the rods are absent Rögner et al. (1990).

The model is able to predict the light attenuation caused by cultures in a considerable range of optical densities and light sources. Besides, the methodology proposed in this work follows a semi-mechanistic calculation procedure that can be generalised to other microorganisms and reactor geometries, whereas other published contributions are merely 
empiric fits or assume that absorption is the only factor for light attenuation. Moreover, this methodology is also capable of predicting spectral composition of light within the photic zone.

In the following sections we will explain the main features of our modelling approach and its assumptions: section 2 exposes the experimental information and underlines how our method can be used in practice combining existing information with novel experiments. Section 3 discusses the results and highlights some interpretations that can be obtained from these analyses. Section 4 contains the conclusions and further outlook of our work.

\section{Light spectrum influence in photosynthetic mechanisms}

As stated before, light spectral composition in a PBR is sometimes not just a given condition, but can be selected and optimised. For an optimal selection of the light source, it is not only important to consider lamps whose emission peaks overlap the cell absorption spectra, but also other factors such as scattering, quantum yield and excitation balance between both types of photosystems Morel (1987).

Moreover, not only the light absorption capacity of the cells but also its efficiency in converting the captured photons into usable energy has to be taken into consideration. In this regard, the action spectrum represents the quantum yield of this efficiency upon light wavelength. It is important to note that the action spectra can vary depending on the pre-illumination conditions Brody and Emerson (1959) or if supplementary light is applied. In the latter case, if cells are not exposed to some background light, the action spectrum can differ greatly from the absorptance spectrum in some wavelengths Fork (1963). In other words, when using a monochromatic light source, the spectrum of the chosen lamp has to provide a balanced amount of quanta for both types of photosystems.

While it is common practice to study how white light affects growth in photosynthetic microorganism cultures, including mechanistic ap- 
proaches for the photo-adaptation phenomenon García-Camacho et al. (2012), less research has been performed on how other types of light sources impact photosynthesis rates and related mechanisms. Specifically in cyanobacteria, some contributions can be found regarding light colour effect on oxygen evolution Ma et al. (2007), redox state of the plastoquinone pool Schuurmans et al. (2014), growth Zavřel et al. (2015) in Synechocystis, biomass composition of Arthrospira platensis Markou (2014) or areal biomass productivity in Chlamydomonas reinhardtii cultures de Mooij et al. (2016). In Zavrel et al. research Zavřel et al. (2015) and Markou contribution Markou (2014), blue light led to lower growth than red in both species, whereas in de Mooij et al. (2016) yellow light promoted the highest productivity. Available irradiance as a function of the remaining wavelengths can shed light on real photosynthesis rates as quanta are absorbed by pigments which have specific absorption spectra on one side while part of the light is scattered in a spectrally dependent way. Particularly in Synechocystis cultures, blue is the most scattered colour and red the least Stramski and Morel (1990), though this phenomenon relies on the type of organism and the aquatic environment Kirk (1994).

Delving deeper in spectral composition of light publications, it must be noted that there are few experimental works which describe the wavelength dependent light distribution along the optical path-length. Measured spectra of remaining light within the photosynthetically ac range at different depths in cyanobacterial cultures of Spirulina platensis Gitelson et al. (1996), suspensions of Chlamydomonas reinhardtii Pottier et al. (2005) and in Microcoleus chthonoplastes mats Jorgensen et al. (1987) are among the few. However, knowing the light field inside PBR cultures would help in designing large-scale flat-type PBRs and predicting growth conditions for maximal photosynthesis rates, e.g. optimal cell density and depth for given illumination conditions and species.

In summary, it is common to model and present photosynthesis as a function of the total white light intensity applied in the system as this approach is sufficient for validating general culture properties. How- 
Chapter 2. Light distribution and spectral composition within

ever, knowing the spectral composition of light is necessary to deeply understand its effect on many photosynthetic processes.

\begin{tabular}{|c|c|c|}
\hline Name & Definition & Unit \\
\hline PAR & $\begin{array}{l}\text { Photosynthetically Active Radia- } \\
\text { tion are those wavelengths in the } \\
\text { range between } 400 \mathrm{~nm} \text { and } 700 \mathrm{~nm}\end{array}$ & \\
\hline G & $\begin{array}{l}\text { A coefficient representing the rel- } \\
\text { ative contribution of scattering to } \\
\text { vertical attenuation }\end{array}$ & \\
\hline $\cos \varphi_{0}$ & $\begin{array}{l}\text { Cosine of the angle of the photons } \\
\text { to the vertical just below the water } \\
\text { surface after refraction }\end{array}$ & \\
\hline$\rho_{C h l a}$ & $\begin{array}{l}\text { Chlorophyll a concentration in the } \\
\text { culture }\end{array}$ & $\mathrm{mg} \operatorname{chla} \cdot \mathrm{m}^{-3}$ \\
\hline $\mathrm{OD}_{\lambda}$ & $\begin{array}{l}\text { Optical density at given wave- } \\
\text { length } \lambda \text { in } \mathrm{nm}\end{array}$ & \\
\hline$\Phi_{j}(\lambda)$ & $\begin{array}{l}\text { Radiant flux absorbed }\left(\Phi_{a}(\lambda)\right) \text {, scat- } \\
\text { tered }\left(\Phi_{b}(\lambda)\right) \text {, attenuated }\left(\Phi_{c}(\lambda)\right) \text {, } \\
\text { transmitted }\left(\Phi_{t}(\lambda)\right) \text { and incident } \\
\left(\Phi_{i}(\lambda)\right)\end{array}$ & $W$ \\
\hline IOP & $\begin{array}{l}\text { Inherent optical property of the cul- } \\
\text { ture }\end{array}$ & \\
\hline$j(\lambda)$ & $\begin{array}{l}\text { Absorption } a(\lambda) \text {, total scattering } \\
b(\lambda) \text { and beam attenuation coeffi- } \\
\text { cient } c(\lambda)\end{array}$ & $\mathrm{m}^{-1}$ \\
\hline att & $\begin{array}{l}\text { Attenuance defined as the negative } \\
\text { common logarithm of the transmit- } \\
\text { tance }\end{array}$ & \\
\hline opd & $\begin{array}{l}\text { Absorbance defined as the negative } \\
\text { common logarithm of the transmit- } \\
\text { tance in the absence of scattering }\end{array}$ & \\
\hline
\end{tabular}


$\rho_{C h l ~ a}$ specific coefficient at wavelength $\lambda$ and where culture is ac$j^{\star}\left(\lambda, E_{d, a c c}\right) \quad$ climated at intensity $E_{d, a c c} ; j$ is a placeholder that either stands for $a, b$ or $c$

AOP

$E_{d}(\lambda, z)$

$E_{d, \operatorname{PAR}}(z)$

$E_{d, \text { acc }}$

$\rho E_{d}(\lambda, z)$

$K_{d}(\lambda, z)$

$\bar{K}_{d}\left(\lambda, E_{d, a c c}\right)$

$\bar{K}_{d, \operatorname{PAR}}\left(E_{d, a c c}\right)$

$\bar{K}_{d, \mathrm{PAR}}^{\star}\left(E_{d, a c c}\right)$
Apparent optical property of the culture

Downward irradiance at the depth $\mathrm{z}$ and at wavelength $\lambda$

Downward irradiance integrated over PAR and at the depth $\mathrm{z}$

Spectral photon flux density of downward irradiance at the wavelength $\lambda$ and depth $\mathrm{z}$

Volume averaged and PAR integrated downward irradiance to which a given PBR culture has been acclimated

Downward attenuation coefficient at the depth $\mathrm{z}$ and at wavelength $\lambda$ Mean downward attenuation coefficient at wavelength $\lambda$ averaged for a culture acclimated at intensity $E_{d, a c c}$

Mean downward attenuation coefficient averaged within PAR range for a culture acclimated at intensity $\mathrm{m}^{-1}$ $E_{d, a c c}$

Chlorophyll a-specific mean downward attenuation coefficient averaged within PAR range for a culture $\mathrm{m}^{2} \cdot \mathrm{mg} \operatorname{chl~} \mathrm{a}^{-1}$ acclimated at intensity $E_{d, a c c}$ $\mu \mathrm{E} \cdot \mathrm{m}^{-2} \cdot \mathrm{s}^{-1} \cdot \mathrm{nm}^{-1}$

$\mu \mathrm{E} \cdot \mathrm{m}^{-2} \cdot \mathrm{s}^{-1}$

$\mu \mathrm{E} \cdot \mathrm{m}^{-2} \cdot \mathrm{s}^{-1} \cdot \mathrm{nm}^{-1}$

$\mu \mathrm{E} \cdot \mathrm{m}^{-2} \cdot \mathrm{s}^{-1}$

$\mathrm{m}^{-1}$

$\mathrm{m}^{-1}$

Table 2.1: List of symbols and abbreviations. 


\subsubsection{Modelling framework definition}

\section{Inherent Optical Properties: definition and measurement}

The two basic IOPs Davies-Colley et al. (1986), the absorption and scattering coefficients, are defined on the basis of an imaginary, infinitesimally thin plane, parallel layer of medium, illuminated at right angles by a parallel beam of monochromatic light. AOPs, such as the different coefficients describing vertical attenuation, are properties of the radiation field depending not only on intrinsic features of the water body but also on the angular distribution of the light within the system as well as the depth.

Further, the photon complex and stochastic interaction in water due to both combined effects of absorption and scattering, does not lead to analytical solutions but in general can be treated only numerically. Photons can be either absorbed or scattered when interacting with matter, whereby in the first case they disappear and are transformed into a different type of energy such as heat or chemical bond excitations. In the case of scattering, the quanta direction and/or energy level is changed. Yet, thanks to inherent optical properties, absorption and scattering spectra of aquatic systems can be characterised.

In these terms, an incident monochromatic light beam, assuming energy conservation and no wavelength change due to scattering process, can be split into absorbed, scattered (both together considered as attenuated) and transmitted radiant flux Kirk (1994):

$$
\Phi_{a}(\lambda)+\Phi_{b}(\lambda)+\Phi_{t}(\lambda)=\Phi_{c}(\lambda)+\Phi_{t}(\lambda)=\Phi_{i}(\lambda)
$$

In practice it is not feasible to carry out measurements on infinitesimally thin layers, which implies the need to relate the absorption, scattering and attenuation coefficients, $a(\lambda), b(\lambda)$ and $c(\lambda)$ respectively, with the measurable absorbance, scatterance and beam attenuance of finite thickness layers. To this purpose, spectrophotometer cuvettes can be used. 
The beam attenuation coefficient $c(\lambda)$ can be linked with the attenuance measured by means of a spectrophotometer through the next equation Kirk (1994):

$$
c(\lambda)=\frac{a t t(\lambda)}{r} \cdot 2.303
$$

where $\mathrm{r}$ is normally in the range of few centimetres in a typical spectrophotometer rectangular cuvette arrangement.

Absorption coefficient $a(\lambda)$ can be calculated in a similar way, although in this case the scattered light can distort the absorption measurement. Once it is reasonable to consider that all attenuation which arises from the scattering effect is small (e.g. by means of an integrative light collection sphere), the optical density or absorbance of the sample may be equated with the absorption coefficient analogously to the case of the attenuation coefficient shown in Equation (2.2):

$$
a(\lambda)=\frac{o p d(\lambda)}{r} \cdot 2.303
$$

Now, from $a(\lambda)$ and $c(\lambda)$, it is straightforward to obtain the scattering coefficient $b(\lambda)$ as:

$$
b(\lambda)=c(\lambda)-a(\lambda)
$$

\section{Estimation of main Apparent Optical Properties}

The beam attenuation coefficient $c(\lambda)$ can give information about the attenuation properties of a water body depending on the wavelength, though it is not sufficient for estimating the real attenuation of light in the medium. To describe attenuation in a given propagation direction $z$, the downward irradiance attenuation coefficient $K_{d}(\lambda, z)$ is usually 
calculated, which is one of the most used AOPs and can appear in the well-known Beer-Lambert law Kirk (2003):

$$
E_{d}(\lambda, z)=E_{d}(\lambda, 0) \cdot e^{-\bar{K}_{d}(\lambda) \cdot z}
$$

As $K_{d}(\lambda, z)$ is an apparent property its determination is in principle only possible if the downward irradiance is measured in situ in the medium. Nevertheless, there have been some attempts to construct semi-empirical formulas that correlate this coefficient with inherent optical properties. By systematic calculation based on radiative transfer theory and Monte Carlo simulations, Phillips and Kirk in 1984 Kirk (1984) found such a correlation, valid for a sun-illuminated water body and given by:

$$
\bar{K}_{d}(\lambda, z)=\frac{1}{\cos \varphi_{0}}\left[a(\lambda)^{2}+G \cdot a(\lambda) b(\lambda)\right]^{1 / 2}
$$

where $G=0.425 \cos \varphi_{0}-0.190, \cos \varphi_{0}$ symbolises the cosine of the zenith angle of refracted photons just beneath the surface, while $a(\lambda)$ and $b(\lambda)$ are the absorption and scattering coefficients, respectively. As $K_{d}(\lambda, z)$ does not significantly depend on the depth within the euphotic range, it can accepted that this parameter remains constant within this region and rewrite it as $K_{d}(\lambda)$. An application example for modelling oceanic water light attenuation using Equation (2.6) can be found in Morel (1991).

In our contribution we will assume that the same physical principles that led to the above relationship apply to the particular case of light propagation within a PBR. In the case of a flat-type PBR placed in a laboratory, illumination is usually perpendicular to the panel planes and hence, the cosine of the zenith angle $\cos \varphi_{0}$ in Equation (2.6) is one. $G$ represents the contribution of scattering with respect to absorption and under these perpendicular illumination conditions equals 0.235. In the case of a flat type PBR placed outside and illuminated by the sun, the position of the sun should be taken into account through the 
zenith angle. An application example for modelling oceanic water light attenuation using Equation (2.6) can be found in Morel (1991).

The combination of Equations (2.5) and (2.6) may in principle be used to estimate light field attenuation for a given wavelength based on previously measured inherent optical properties of the organism of study. Nevertheless, radiometric measurements used to evaluate the light field in a water body are often not specifically sensitive to wavelength and as a result simply collect those photons within the so-called photosynthetic active radiation (PAR) (about 400 to $700 \mathrm{~nm}$ ), treating them as a single value. In such situation it is more appropriate to use AOPs that represent the whole PAR Kirk (1994), by accepting the hypothesis that the validity of the Beer-Lambert relation, displayed in the expression (2.5), can be extended to the whole PAR range:

$$
E_{d, \mathrm{PAR}}(z)=E_{d, \mathrm{PAR}}(0) \cdot e^{-\bar{K}_{d, \mathrm{PAR}} \cdot z}
$$

Besides, PAR irradiance is given as:

$$
E_{d, \operatorname{PAR}}(z)=\int_{400}^{700} E_{d}(\lambda, 0) \cdot e^{-\bar{K}_{d}(\lambda) \cdot z} d \lambda
$$

Combining Equations (2.7) and (2.8) and rearranging terms we find the following expression for $\bar{K}_{d, \mathrm{PAR}}$ :

$$
\bar{K}_{d, \mathrm{PAR}}=-\frac{1}{z} \ln \left(\int_{400}^{700} \rho E_{d}(\lambda, 0) \cdot e^{-\bar{K}_{d}(\lambda) \cdot z} d \lambda\right)
$$

where $\rho E_{d}(\lambda, 0)$ represents the spectral photon flux density that measures the relative contribution of the different wavelengths to $E_{d}(\lambda, 0)$, commonly referred to as the lamp emission spectrum. Although the depth variable $\mathrm{z}$ appears in the former relation, the $\bar{K}_{d \text {,PAR }}$ value remains basically constant up to depths in which the spectral composition of light has substantially changed in comparison with that of incident light. This change in spectral composition is due to the fact that photons corresponding to green wavelengths are less frequently absorbed. At larger depths, thus, the $\bar{K}_{d \text {,PAR value will converge towards }}$ the smaller attenuation coefficient of monochromatic green light. 


\section{Calculation of the average light intensity}

When dealing with microorganisms the analysis of their optical properties is much more complicated, as many other factors must be taken into consideration: the growth medium, the fitness of the culture and even the fact that cells must be able to acclimate to varying light intensities and changes in light spectrum. This latter property specially makes the question much more difficult for a mathematical treatment, as IOPs keep memory of the light conditions which cells have been previously subjected in such a way that in essence: $a=a\left(\lambda, E_{d}\left(t^{\prime}, \lambda^{\prime}\right)\right)$ and $b=b\left(\lambda, E_{d}\left(t^{\prime}, \lambda^{\prime}\right)\right) \forall t^{\prime} \in\left[t-t_{a c c}, t\right], \lambda^{\prime} \in \mathrm{PAR}$. This expression reflects the fact that the IOP (and thus all related AOPs) depend on the intensity, spectral distribution and time evolution of light during the immediately previous acclimation time window, which ranges from hours to days Kwon et al. (2012) and is represented by $t_{a c c}$.

Within a PBR running under stationary conditions the question can be substantially simplified considering the average light intensity as an indicator of bioengineering properties. Such approach has been repeatedly used since 1962 when it was applied for estimating growth in dense cultures Rabe and Benoit (1962). When cells are moving along the whole optical path-length and are homogeneously distributed, it is reasonable to accept that all are exposed in time-average to the same intensity and light spectrum which equals the mean value of light irradiance within the PBR volume. Given that optical conditions are constant during a sufficient lapse of time (at least longer than $t_{a c c}$ ), cells will physiologically adapt to this, a priori unknown, average light intensity Molina Grima et al. (1994). Our model will develop this idea, though it should be noted that for cells growing in fluctuating light conditions, photosynthetic performance will additionally depend on the dynamics of the fluctuating light regime, that is, not only on the overall time exposure to light and darkness but also on the switch frequency Molina Grima et al. (1999). 
To correlate the experimental conditions in which the IOPs $a$ and $b$ are measured or characterized with a given PBR experiment we express them as follows:

$$
j\left(\lambda, E_{d, a c c}\right)=\rho_{C h l a} \cdot j^{\star}\left(\lambda, E_{d, a c c}\right) \quad j=a, b
$$

where $E_{d, a c c}$ is a constant acclimation downward PAR light intensity to which cells were exposed during a time interval $t \geq t_{a c c}$ before measurement took place, $a^{\star}\left(\lambda, E_{d, a c c}\right)$ and $b^{\star}\left(\lambda, E_{d, a c c}\right)$ are chlorophyll a-specific absorption and scattering coefficients corresponding to cells which have been acclimated to these intensities. Equation (2.10) also assumes that the IOPs are in a linear relationship with the amount of chlorophyll a (hereafter referred to as chl a) present in the PBR suspension. Similarly, for attenuation coefficients it is possible to define total and chl a-specific magnitudes. Given that we have characterized our cells in a sufficiently representative range of acclimation intensities $\left\{E_{d, a c c_{1}}, E_{d, a c c_{2}} \cdots E_{d, a c c_{n}}\right\}$ we can, through interpolation, construct functions $a\left(\lambda, E_{d, a c c}\right)$ and $b\left(\lambda, E_{d, a c c}\right)$ that allow us to calculate the IOPs for any given intensity within that range. Then, using (2.6) it is possible to obtain the corresponding function that represents the downward attenuation coefficient:

$$
\begin{aligned}
& \bar{K}_{d}\left(\lambda, E_{d, a c c}\right)=\rho_{C h l a} \cdot \bar{K}_{d}^{\star}\left(\lambda, E_{d, a c c}\right)= \\
& \rho_{C h l a} \sqrt{a^{\star}\left(\lambda, E_{d, a c c}\right)^{2}+G \cdot a^{\star}\left(\lambda, E_{d, a c c}\right) b^{\star}\left(\lambda, E_{d, a c c}\right)}
\end{aligned}
$$

for any given value of the volume-average irradiance intensity. Rewriting Equation (2.6) and taking into account expression (2.10):

$$
\bar{K}_{d, P A R}\left(E_{d, a c c}\right)=-\frac{1}{z} \ln \left(\int_{400}^{700} \rho E_{d}(\lambda, 0) \cdot e^{-\rho_{C h l a} \cdot \bar{K}_{d}^{\star}\left(\lambda, E_{d, a c c}\right) \cdot z} d \lambda\right)
$$

In a usual PBR experiment in which cells do have time to acclimate to the long-term conditions, the average light intensity in the reactor must be found as the solution of a non-linear equation. To illustrate the idea, 
in the particular case of a flat plate reactor with one-sided illumination from one single planar light source we can for instance calculate the average light intensity as:

$$
\begin{aligned}
E_{d, a c c} & =\frac{E_{d, \operatorname{PAR}}(0)}{L} \int_{0}^{L} e^{-\bar{K}_{d, \mathrm{PAR}}\left(E_{d, a c c}\right) \cdot z} d z \\
& =E_{d, \mathrm{PAR}}(0) \frac{1-e^{-L \cdot \bar{K}_{d, \mathrm{PAR}}\left(E_{d, a c c}\right)}}{L \cdot \bar{K}_{d, \mathrm{PAR}}\left(E_{d, a c c}\right)}
\end{aligned}
$$

which can be solved numerically for the unknown value $E_{d, a c c}$ of the average light intensity equal to the acclimation intensity in the PBR, whose depth is $L$.

Expression (2.13) can be understood as a self-consistency condition between the average intensity of the light field inside the PBR and the resulting attenuation coefficient, but we would like to stress that this particular form is valid for the case of a one-side illuminated flat panel (or for a two-side illumination set-up where incident intensity would be half for maintaining equivalent conditions). For other geometric configurations (e.g. multiple panel arrangements, tubular PBRs) the concept remains the same, but $E_{d, a c c}$ will have a different formal expression. In any case, our methodology is easily extensible to these other cases. In the following, we will refer to this approach as Auto-consistent Field Approximation Algorithm (AFA).

\subsection{Materials and Methods}

\subsubsection{Validation strategy and modelling scheme implementation}

To test the predictability of our method, we measured the IOPs of two similar organisms, wild-type (WT) and Olive strains of Synechocystis in tightly controlled PBR conditions Kwon et al. (2012) to calculate their specific attenuation coefficients, $\bar{K}_{d}^{\star}\left(\lambda, E_{d, a c c}\right)$. We then used these coefficients to deduce the actual attenuation of light in cultures of the same 


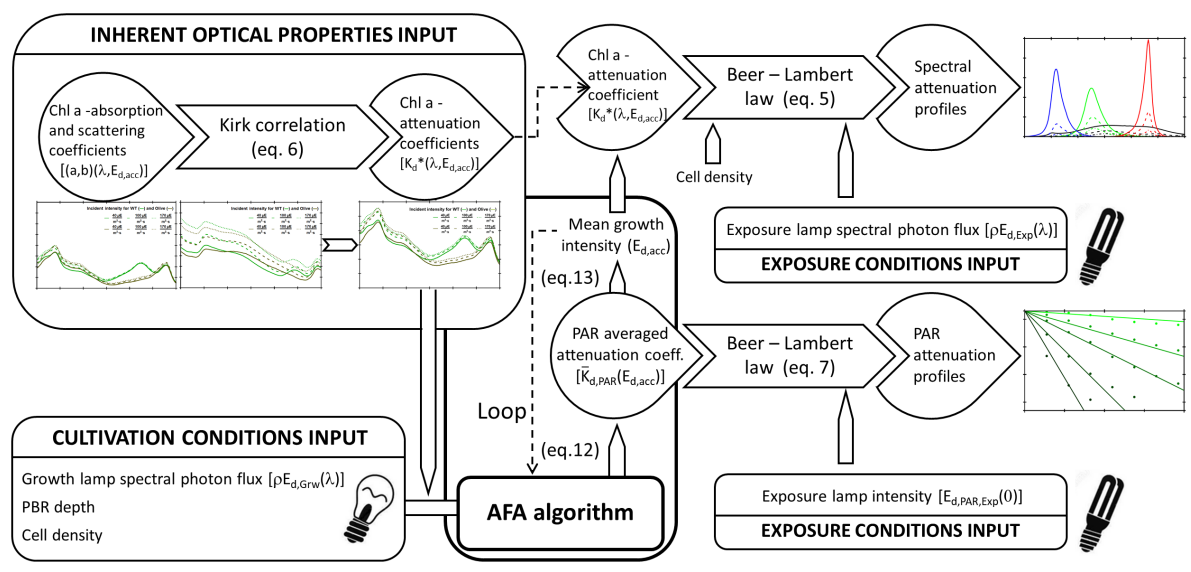

Figure 2.1: Summarised modelling scheme to obtain in silico PAR attenuation profiles and spectral ones.

organisms characterized by Lea-Smith and his co-workers in different experiments Lea-Smith et al. (2014), and compare them with the actual measured attenuation coefficients. The in silico work was integrally performed in MATHEMATICA ${ }^{\circledR} 10.4$.

\section{Measurement of the IOPs spectra and calculation of the attenuation coefficient function}

Before taking the optical properties measurements, Synechocystis cultures were grown in stable conditions so that they got acclimatised to mean irradiance. Cells were grown in a 5 litres flat-bed photobioreactor with a surface-to-volume-ratio of $50 \mathrm{~m}^{-1}$ and a depth of $4 \mathrm{~cm}$ at constant $\mathrm{pH}$ of 7.0 and temperature value of $30^{\circ} \mathrm{C}$ in continuous operation after they were inoculated Kwon et al. (2012). Cell density was maintained constant under turbidostatic process control. Cells were cultivated for at least 48 hours till a constant growth rate was established.

We analysed Synechocystis cultures, namely wild-type strains and the truncated antenna Olive mutants to obtain their specific absorption and scattering coefficients in stable PBR conditions to ensure that organisms are acclimated to the same intensity in enough time. To this purpose, 
absorbance and attenuation spectra within the PAR range were measured at every nanometre after cultivating cells at three different incident light intensities, 40,100 and $170 \mu \mathrm{mol}$ photons $\cdot \mathrm{m}^{-2} \cdot \mathrm{s}^{-1}$ of cool white LED lamp, covering the usual range of intensities that cells may encounter inside a PBR. After stabilization of the culture at an $O D_{750}$ value of 0.5 , a sample was taken to measure absorptance and attenuance of the cells in the different conditions.

Optical measurements of the samples were performed by means of a Shimadzu UV2450 UV-vis spectrophotometer equipped with an integrating sphere for absorbance measurements. The latter device is a double-beam system integrating sphere (ISR-2200) whose internal diameter is $60 \mathrm{~mm}$ with $\mathrm{BaSO}_{4}$ inside coating. The culture samples were previously diluted to reduce effects of self-shading and multiple scattering, keeping the maximum optical thickness at $400 \mathrm{~nm}$, well below 0.3, a threshold consistent with a given criterion Van de Hulst (1957). This guarantees that the measured optical coefficients are inherent rather than hybrid optical properties Preisendorfer (1961). Finally, the total scattering coefficient, $b$, for all angles (except for the acceptance angle of the photomultiplier tube 0 to 5 degrees) was determined by subtracting the beam attenuation, $c$, from the true absorption coefficient, $a$.

From these measurements and by means of Equations (2.2), (2.3) and (2.4) a set of spectral absorption and scattering coefficient-functions, $a\left(\lambda, E_{d, i}\right)$ and $b\left(\lambda, E_{d, i}\right)$, where $i=40,100,170$, and their corresponding chl a-specific functions $a^{\star}\left(\lambda, E_{d, i}\right)$ and $b^{\star}\left(\lambda, E_{d, i}\right)$, where $i=40,100,170$ were derived. The results are shown in Figure 2.4.

However, it is important to note that the real acclimation intensities of the cultures are lower than the referred to above incident intensity values of either 40,100 or $170 \mu \mathrm{mol}$ photons $\cdot \mathrm{m}^{-2} \cdot \mathrm{s}^{-1}$. To find the correct acclimation intensities an iterative procedure was followed using our proposed AFA-algorithm. A summarised scheme of the whole calculation process is shown in Figure 2.1. There it can be seen that the methodology transforms the required input, i.e. lamp characteristics 
during growth phase, cell-density, optical path-length and attenuation function, into the mean acclimatisation intensity and PAR averaged attenuation coefficient. This is done in a close loop between these two magnitudes. Afterwards, both can be used to obtain light field distribution using Beer-Lambert law. If it is desired to apply the attenuation coefficient function with spectral resolution $\bar{K}_{d}\left(\lambda, E_{d, a c c}\right)$, different attenuation results will be obtained for each wavelength and intensity, whereas in the case of the PAR related coefficient $\bar{K}_{d, P A R}\left(E_{d, a c c}\right)$, the attenuation is just a single representative value and the coefficient is directly estimated by means of the algorithm solution. Though in any case, the Beer-Lambert equation remains the same and just the coefficient has a different meaning. Moreover, the method allows one to estimate culture attenuation with cells owning previous optical properties but exposed to different illumination conditions. However, we will normally be interested in assessing the light field in a PBR system where cells are growing, so exposure and cultivation conditions (including lamps) will be the same.

This procedure can also be applied to estimate the mean irradiance in our PBR set-up at different lamp intensities by updating the total attenuation and mean irradiance and checking its convergence:

1. First, $\bar{K}_{d, \text { PAR:0 }}$ is calculated assuming that the acclimation intensities in the experiments were the nominal set "0" $=\{40,100,170\}$, $\mu \mathrm{mol}$ photons $\cdot \mathrm{m}^{-2} \cdot \mathrm{s}^{-1}$. To do so, we just need to substitute these values in Equation (2.12), which integrates the reconstructed attenuation spectra (Figure 2.6) at the given intensities to deliver the $\bar{K}_{d, \text { PAR:0 }}$ PAR attenuation coefficients.

2. Thanks to these parameters, we can estimate in a direct way the corresponding set of acclimation intensities in the PBR by directly substituting $\bar{K}_{d \text {,PAR:0 }}$ in Equation (2.13). In this manner, a new set of acclimation intensities "1" $\left\{E_{d, a c c, 40: 1}, E_{d, a c c, 100: 1}, E_{d, a c c, 170: 1}\right\}$ is obtained.

3. Taking into consideration this new set of irradiance values, the new $\bar{K}_{d, \text { PAR:1 }}$ is calculated and, solving again the same equations 
for each of the three experiments, a further set of acclimation intensities "2" $\left\{E_{d, a c c, 40: 2}, E_{d, a c c, 100: 2}, E_{d, a c c, 170: 2}\right\}$ is obtained.

4. One can see that in just a couple of iterations the acclimation intensities converge to a stable value, which will be considered the final acclimation intensities that are used to reconstruct the organism specific attenuation coefficient function: $\bar{K}_{d}^{\star}\left(\lambda, E_{d, a c c}\right)$.

5. The obtained final average irradiance, given as a percentage value, with respect to our three studied lamp intensities are $48 \%, 49 \%$ and $60 \%$ for WT and $59 \%, 60 \%$ and $61 \%$ for Olive. The higher value for the WT cultures grown at $170 \mu \mathrm{mol}$ photons $\cdot \mathrm{m}^{-2} \cdot \mathrm{s}^{-1}$ is due to a considerable reduction in the chlorophyll concentration and this causes the total attenuation to drop. The estimated irradiance fits well with previous estimation of other authors for the WT strain Zhang et al. (2010).

It is remarkable that for calculating the light field in any further condition, the same approach is used: from incident irradiance values, PAR coefficients first and related average irradiance values are obtained in a self-consistent way in a few iterative steps.

\section{Application of the derived attenuation function to experiments}

In a completely independent way, Lea-Smith and his co-authors measured the light field of WT and Olive Synechocystis cultures grown in the same optical environment but momentarily exposed to a variety of conditions (to in situ measure the light distribution), including different types of light sources.

With our proposed approach it is now possible to use the reconstructed function $\bar{K}_{d}^{\star}\left(\lambda, E_{d, a c c}\right)$ together with the rest of the required input information to first estimate $\bar{K}_{d, \mathrm{PAR}}\left(E_{d, a c c}\right)$ and thanks to it, the attenuation profiles.

To benchmark our in silico predictions with the experiments described in the referenced work, several specific parameters, namely the values 
of $\rho_{C h l a}$ and $E_{d, a c c}$ must be additionally deduced, which requires some knowledge and analysis of how the measurements were performed. Moreover, as light field samples were linked to $\mathrm{OD}_{750}$ values, the referenced chl a concentration for WT and Olive cultures per $\mathrm{OD}_{750}$ unit in that contribution is used, which is 5,400 and 5,300 $\mathrm{mg}$ chl a for WT and Olive, respectively.

\section{Description of the experimental set-up and deduction of the relevant parameters from the published measurement results}

As described in the referenced work Lea-Smith et al. (2014), cell suspensions of around $5 \mathrm{~cm}$ were first grown in conical flasks under 120 $\mu \mathrm{mol}$ photons $\cdot \mathrm{m}^{-2} \cdot \mathrm{s}^{-1}$ halogen white light. By means of an elemental geometric analysis which is dependent on the shape of the flask, we have deduced an equivalent optical path-length of $4 \mathrm{~cm}$. Anyhow, these types of approximations, due to our lack of exact knowledge about how the experiments were made, are necessarily prone to a certain degree of uncertainty.

After reaching the desired $\mathrm{OD}_{750}$ values and in order to perform the attenuation trials, the cells were transferred to an $11 \mathrm{~cm}$ custom made apparatus used for measuring light penetration at different depths (in which several light detectors where located every $11 \mathrm{~mm}$ up to 110 $\mathrm{mm}$ ). For our analysis we will depart from the consideration that since the attenuation experiments were done shortly after the cells were transferred to the new vessel, the acclimation intensity of the cells, and thus their spectral $K_{d}^{\star}$ function corresponds to the acclimation intensity in the conical flask in which they have been grown.

The Synechocystis attenuation data set is composed of light intensity values at increasing depths for the strains here studied plus two extra antenna mutants (not assessed in this contribution), all of them exposed to white LED light at 5 different $\mathrm{OD}_{750}$ values $(0.1,0.5,1.0$, $2.5,5.0)$ and at three different irradiance values $(500,1,000$ and 2,000 $\mu$ mol photons $\cdot \mathrm{m}^{-2} \cdot \mathrm{s}^{-1}$ ), summing a total of 15 white light experiments. Additional blue, green and red LED light trials were carried out at an 
$\mathrm{OD}_{750}$ value of just 1.0 and $1,000 \mu \mathrm{mol}$ photons $\cdot \mathrm{m}^{-2} \cdot \mathrm{s}^{-1}$ of light intensity. The emission spectra of the four LED lamps used in the different experiments are represented in Figure 2.2.

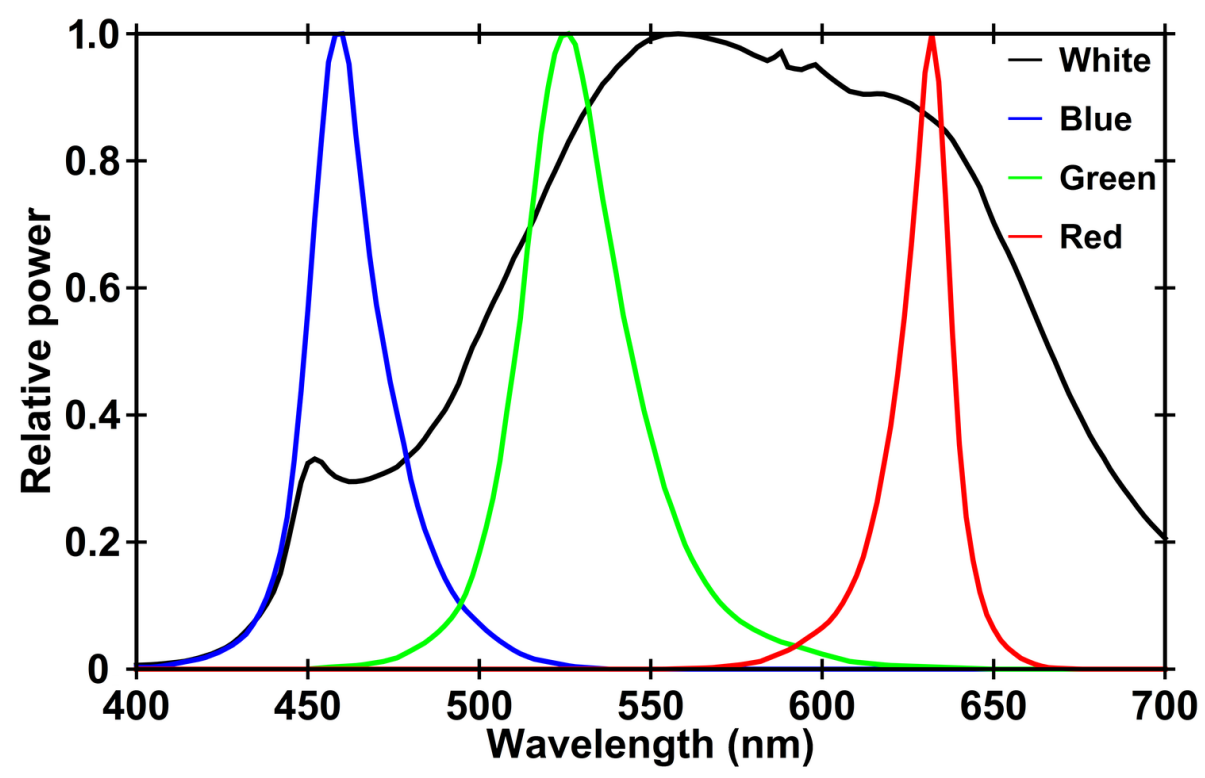

Figure 2.2: Emission spectra in terms of relative power of the LED lamps used for the attenuation trials. Graph colours represent each LED characteristic colour (blue, green and red), whereas black curve corresponds to the white LED.

It is worth stressing the assumption that cells didn't have enough time to adapt to the new environment and consequently they simply expressed their optical properties arising from the previous growth environment in the flasks and not from the attenuation experiment conditions in the custom made attenuation measurement device. Indeed, though cultures were exposed to three different irradiance levels -500 , 1,000 and $2,000 \mu \mathrm{mol}$ photons $\cdot \mathrm{m}^{-2} \cdot \mathrm{s}^{-1}$ - they presented a similar attenuation coefficient in the original work for any given cell density, supporting our hypothesis.

Other differences in the growth conditions among both laboratory setups should be discussed, specifically those connected with the differences in the spectral characteristics of the light sources used. The cells grown in Lea-Smith et al. (2014) were cultivated with halogen lamps 
whereas for estimating Synechocystis IOPs, we employed a cool white LED light. This may in principle generate differentiated optical properties in the cells, but both lamps spectra have quite a wide band of action in the PAR range, a similar shape, and they can mainly be distinguished by the blue peak of the cool white LED spectrum. As Synechocystis cells have the capability to reorganise the photosynthesis apparatus for balancing light input in order to seek optimal growth, we would expect a similar light absorption and scattering profile of the cells cultured under the light of these two lamps. A distinct outcome would be expected if a light source with non-equivalent emission spectrum profile would be employed. In fact, in marine Synechocystis cultures (Synechocystis sp. BCC010, Banyuls collection) grown under blue or green light, the measured spectra had a slightly different shape and half of the amplitude of those corresponding to cells cultivated under similar conditions with white light Stramski and Morel (1990).

We thus conclude that the attenuation coefficients measured in the attenuation assays by Lea-Smith and co-workers should correspond to the acclimation intensity within which the cells were grown in the conical flasks. To find these intensities from the original experiments, we solved, for each of the conditions, the non-linear equation that allows us to obtain such intensity self-consistently. The corresponding expression for the acclimatisation irradiance is given in Equation (2.13), with $E_{d}(0)=120 \mu \mathrm{mol}$ photons $\cdot \mathrm{m}^{-2} \cdot \mathrm{s}^{-1}, L=4 \mathrm{~cm}$ and $\rho_{C h l a}$ deduced from the corresponding $\mathrm{OD}_{750}$ value in each experiment.

\subsubsection{Linearity check of the $K_{d}$ vs. $\mathrm{OD}_{750}$ relationship}

In our light field model, we ultimately relate a given value of an $\mathrm{OD}_{750}$ to which cells have grown to a given chl a concentration and subsequently to a downward attenuation coefficient. In this regard, it is important to assess the limits of the validity of such an assumption. What respects the $\mathrm{OD}_{750}$ vs. chl a relationship, in Figure 2.3 it is shown that in our experiments the relationship between chl a and $\mathrm{OD}_{750}$ remains 
approximately linear for the studied OD range in both WT and Olive strains grown at a nominal PBR intensity of $100 \mu \mathrm{mol}$ photons $\cdot \mathrm{m}^{-2} \cdot \mathrm{s}^{-1}$.

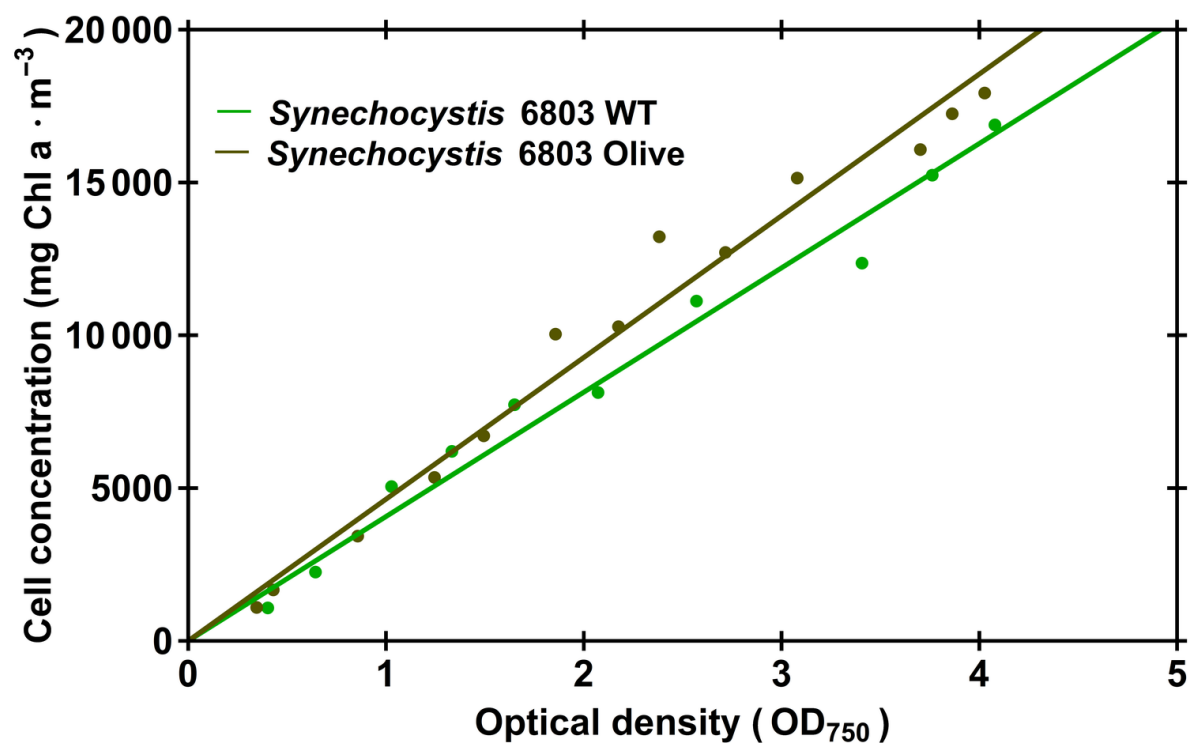

Figure 2.3: Relationship between chlorophyll a and $\mathrm{OD}_{750}$ value of both strains (WT in green colour and Olive in brown one) grown at incident $100 \mu \mathrm{mol}$ photons $\cdot \mathrm{m}^{-2}$. $s^{-1}$. Dots represents experimental data and the line represents the lineal regression.

There are, apart from these analysed experiments, further empirical data that support our hypothesis of a linear behaviour between attenuation coefficient and cell density in dense cultures. In this context we may mention the contributions of Zhang and co-workers, that in Synechocystis cultures studied the ratio of cell concentration as dry weight to the PAR attenuation coefficient Zhang et al. (2010). Gitelson and coworkers worked with Spirulina platensis cultures, where the relationship between chl a concentration and spectral attenuation coefficient were found to be almost constant for a wide range of cell concentrations Gitelson et al. (1996). In both mentioned contributions, we can find the maximum reported cell concentration equivalent up to $4 \mathrm{OD}_{750}$ units, practically covering the same range of densities as in our research.

Moreover, there is one publication where poly- and monochromatic light attenuation in dense and ultra-dense cultures of the green alga Chlorella vulgaris were analysed Yun and Park (2001). It was reported 
that attenuation coefficients augment linearly with the cell concentration up to values of around $300 \mathrm{~m}^{-1}$, which is in agreement with our modelling hypothesis. Above this value the relationship tends to get saturated.

\subsection{Results and discussion}

\subsubsection{Optical spectra and analysis of the resulting IOPs}

As a first outcome, the chl a absorption for both strains resemble each other significantly in shape and amplitude as it is shown in Figure 2.4 (A). Olive spectra lack the absorption of phycocyanin pigment in the orange range and have a slightly larger absorbance in the blue band due to a somewhat higher carotenoid presence, as was already reported Kwon et al. (2013). Phycobilisomes appear not to be dismantled at moderate light intensities within our irradiance range, as the absorption peak of phycocyanin doesn't progressively drop as it does in the case of the marine Synechocystis WT strain Stramski and Morel (1990). The fact that chl a-specific absorption spectra show a constant absorption peak at $675 \mathrm{~nm}$ is expected, since absorption in this band is mainly caused by chl a itself in Synechocystis and to a much lesser extent due to allophycocyanin pigment Simis and Kauko (2012). Indeed, all spectra have a local maximum value of around $0.022 \mathrm{~m}^{2} \cdot \mathrm{mg} \mathrm{chl} \mathrm{a}^{-1}$ at 675 $\mathrm{nm}$ for Olive and a similar one of $0.020 \mathrm{~m}^{2} \cdot \mathrm{mg} \mathrm{chl} \mathrm{a}^{-1}$ for the WT Synechocystis. In Stramski and Morel (1990) similar values were reported for this wavelength.

Scattering spectra, shown in Figure 2.4 (B), are likewise practically identical in both studied strains and have local minima close to the absorption peaks. The shift of the peaks to slightly shorter wavelengths with respect to the absorption ones can be explained by the anomalous dispersion theory Morel and Bricaud (1981b). Furthermore, the likeness in their shape is an anticipated outcome as both strains have comparable cell diameters Lea-Smith et al. (2014), similar chl a amount and 


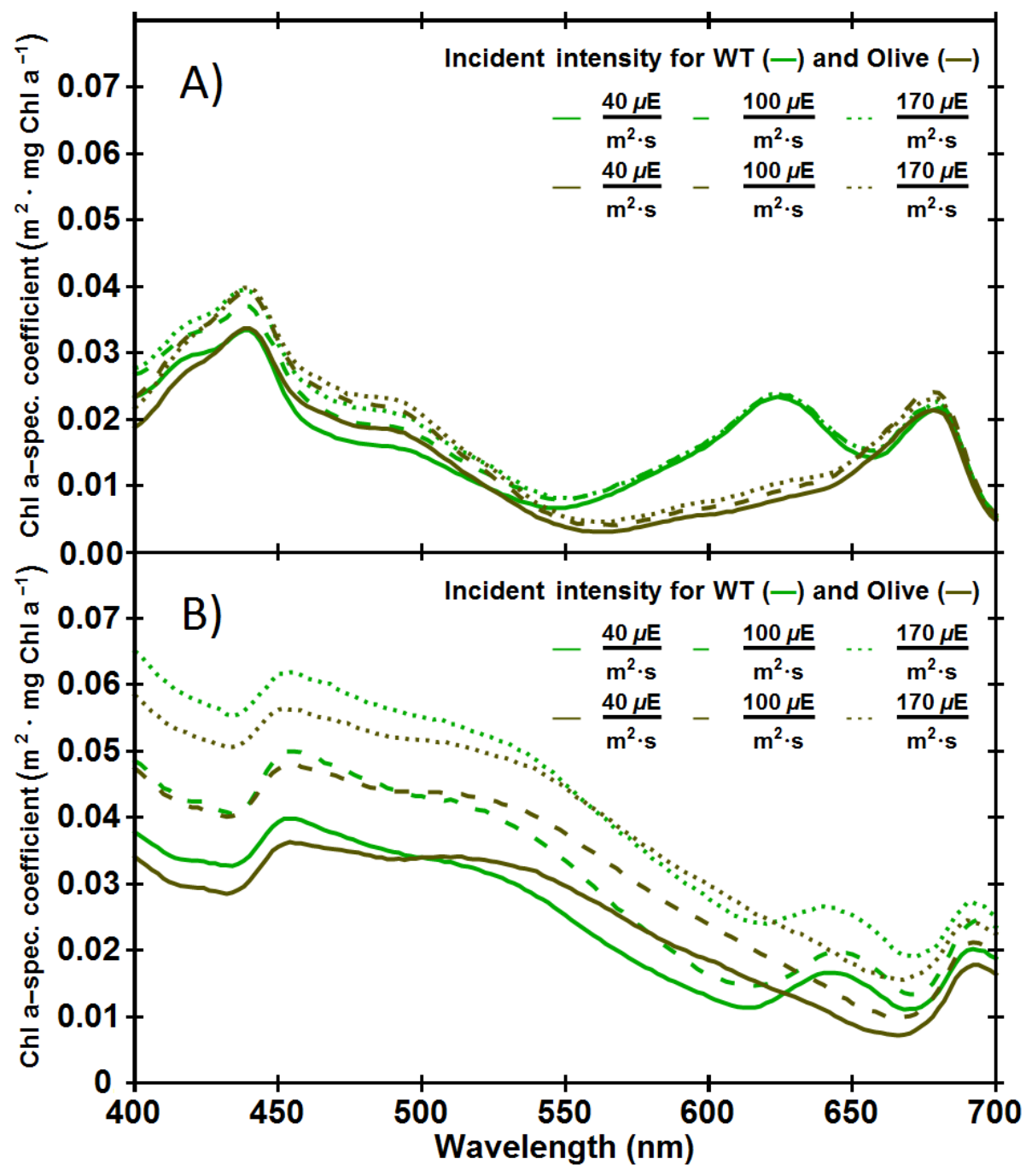

Figure 2.4: (A) Absorption $a^{\star}\left(\lambda, E_{d, i}\right)$ and (B) total scattering $b^{\star}\left(\lambda, E_{d, i}\right)$ chl $a$ specific coefficients within PAR waveband of wild-type and Olive strain (green and brown colour, respectively) grown at incident light intensities of 40, 100 (dashed) and 170 (dotted) $\mu$ mol photons $\cdot m^{-2} \cdot s^{-1}$ in a 4-cm flat-type photobioreactor. 
pigment composition Kwon et al. (2013). It is noteworthy that the ratio between scattering and absorption coefficients at a given intensity in both strains is much lower than in the Synechocystis marine strain, because in the latter, chlorophyll a content per cell was 5 to 8 times lower and thus its absorption capacity was also lower.

Absorption and scattering in $\mathrm{m}^{-1}$ units show a different behaviour with respect to their chlorophyll referenced magnitudes: scattering remains practically constant for all intensities, whereas absorption coefficients slightly decline with increasing intensities, especially at an irradiance level of $170 \mu \mathrm{mol}$ photons $\cdot \mathrm{m}^{-2} \cdot \mathrm{s}^{-1}$.

To quantitatively assess the relative importance of scattering in both studied strains, from Equation (2.6) it can be easily deduced that the term $G \cdot b(\lambda) /(a(\lambda)+G \cdot b(\lambda))$ quantifies the influence of scattering in total attenuation as its complement to one, $a(\lambda) /(a(\lambda)+G \cdot b(\lambda))$, would approach unity in an hypothetical, infeasible "absorption without scattering" scenario. According to this analysis, within PAR 60\% to $90 \%$ of attenuation is due to absorption for both strains, see (Figure 2.5). As expected, there is an exception in the green band where absorption is much lower due to the Synechocystis lack of specific absorption pigments for this band. In addition, higher light intensities lead in both strains to an increased scattering contribution (though scattering coefficient itself keeps constant) at the expense of a lower absorption participation because at higher irradiance values, cells pigment concentration is in generally decreased with the exception of carotenoids, so cells do have less chances to capture photons, meanwhile they have a higher probability to be scattered along the optical path-length. It has to be noted that scattering itself doesn't contribute to the disappearance of photons as they can only be taken out of the medium by the biomass or the water body absorption but it can effectively contribute to an increased light attenuation due to longer optical path-lengths.

Furthermore, averaging along the PAR range and taking into consideration the emission spectrum of the different LED sources, the overall influence of photon scattering/absorption as a percentage value can be 


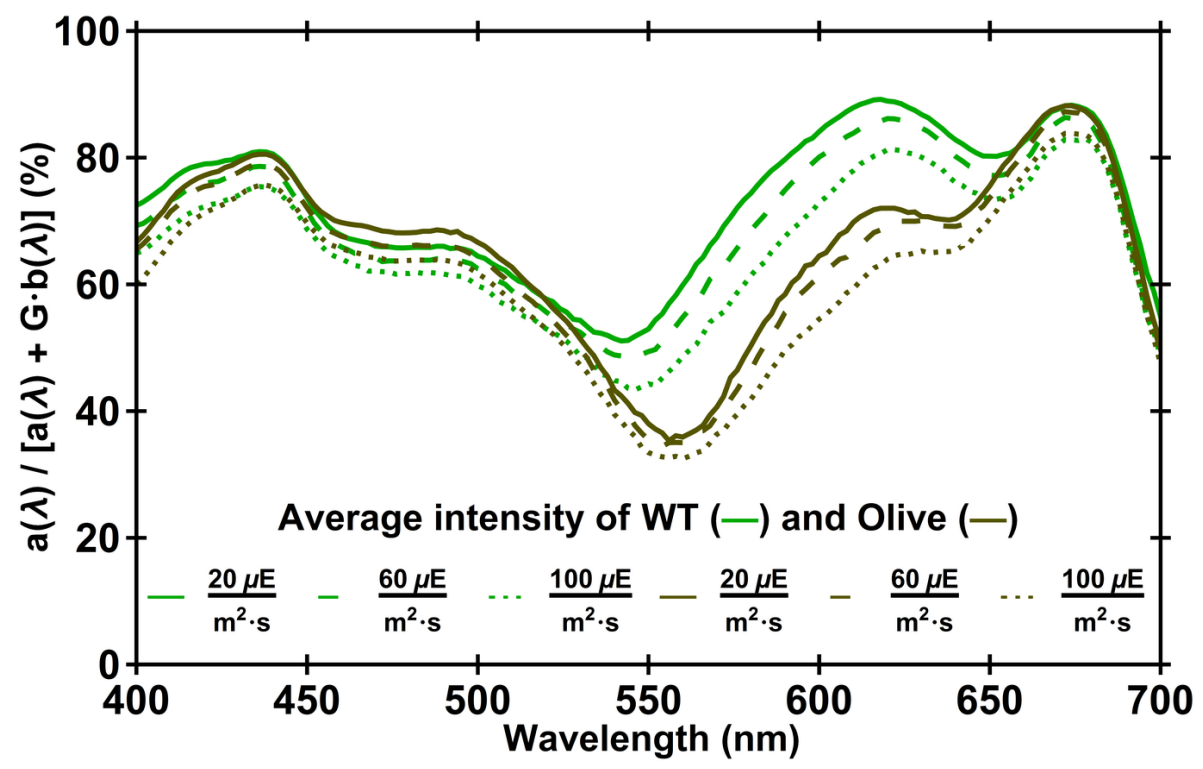

Figure 2.5: Modelled photon absorption contribution of wild-type and Olive strain (green and brown colour, respectively) grown at average light intensities of 20,60 (dashed) and 100 (dotted) $\mu$ mol photons $\cdot m^{-2} \cdot s^{-1}$ for each wavelength within PAR range following Kirk's formula.

estimated. For white LED source illumination, approximately one third of attenuation depends on scattering in the WT strain, while in Olive this value is slightly higher. In the propagation of green light, scattering shows a stronger influence (50\%), while the opposite occurs in red light attenuation (20\%). As light intensities increase, scattering tends to play a more significant role, especially for the WT strain, though this increase is not remarkable in the range of studied irradiance (data not shown).

\subsubsection{Attenuation profiles for white light exposure}

In order to estimate attenuation within the cultures, chlorophyll-specific spectral coefficient functions $\bar{K}_{d}^{\star}\left(\lambda, E_{d, a c c}\right)$ have to be calculated first. This function at the three acclimation intensities display a similar shape as the absorption spectra, but with higher values at the blue band due to the increased contribution of scattering in this range (Figure 2.6). In 


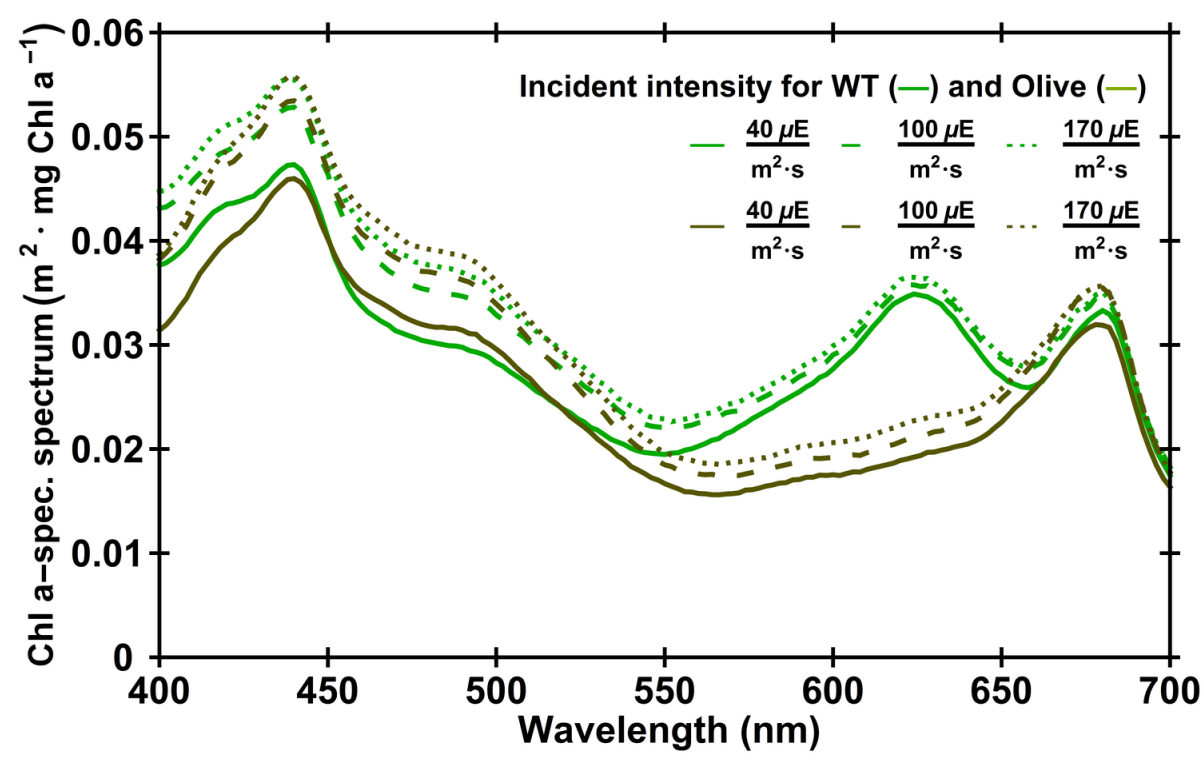

Figure 2.6: Modelled chlorophyll-specific downward attenuation function $\bar{K}_{d}^{\star}\left(\lambda, E_{d, i}\right)$ for wild-type and Olive strain (green and brown colour, respectively) at the incident irradiance values of 40, 100 (dashed) and 170 (dotted) $\mu$ mol photons . $m^{-2} \cdot s^{-1}$.

both strains, maximum values at $440 \mathrm{~nm}$ are comprised between 0.045 and $0.055 \mathrm{~m}^{2} \cdot \mathrm{mg} \mathrm{chl} \mathrm{a}^{-1}$. It might seem that as the chlorophyll-specific attenuation coefficients increase somewhat upon irradiance, total attenuation should follow this trend. But it has to be noted that as light intensity increases, chlorophyll concentration in the cell drops and so does the total attenuation. By multiplying the chlorophyll-specific attenuation coefficients by the chlorophyll amount at each light intensity, the total downward attenuation coefficient can be calculated. In this regard, total attenuation coefficients in $\mathrm{m}^{-1}$ get gradually reduced with increasing intensities (data not shown) due to smaller absorption coefficients. Besides, the attenuation spectra have values between $70-90 \mathrm{~m}^{-1}$ in both strains at $440 \mathrm{~nm}$, which are quite close to the ones reported for Spirulina platensis Gitelson et al. (1996), a cyanobacterium with comparable pigment composition and absorption spectra shape, given similar cell chlorophyll concentrations. 

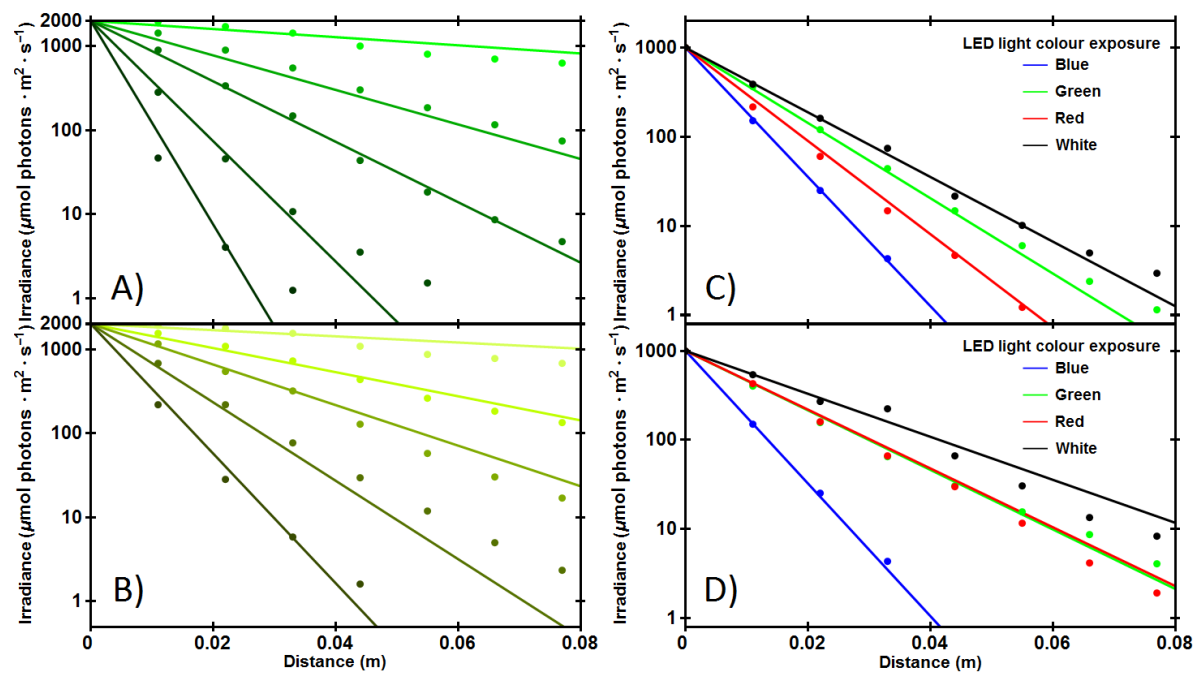

Figure 2.7: Left panel: Light attenuation profiles of Synechocystis $W T(A)$ and Olive (B) strain cultures exposed to 2,000 $\mu$ mol photons $\cdot m^{-2} \cdot s^{-1}$ of white LED light at five different $\mathrm{OD}_{750}$ concentrations $(0.1,0.5,1.0,2.5$ and 5.0) are depicted. Dots are the original source samples Lea-Smith et al. (2014) and lines the simulation outcome. Darker colours correspond to denser suspensions. Right panel: Light attenuation profiles of Synechocystis WT (C) and Olive (D) strain cultures exposed to 1,000 umol photons $\cdot m^{-2} \cdot s^{-1}$ of four different LED lamps at an $\mathrm{OD}_{750}$ concentration of 1.0 are shown. Dots are the original source samples. Graph colours represent each LED characteristic colour (blue, green and red), whereas black curve corresponds to the white LED.

Following the corresponding calculation pipeline summarised in Figure 2.1, attenuation profiles (intensity vs. depth within the measurement assay) are obtained. They correspond to attenuation for cells grown under white halogen light and momentarily exposed to different white LED light intensities at several $\mathrm{OD}_{750}$ values. The experimental irradiance weighted attenuation coefficient of five different cell-density concentration samples $\left(0.1,0.5,1.0,2.5\right.$ and $\left.5.0 \mathrm{OD}_{750}\right)$ at an incident irradiance of $2,000 \mu \mathrm{mol}$ photons $\cdot \mathrm{m}^{-2} \cdot \mathrm{s}^{-1}$ are hereby compared with the attenuation coefficient (or more rigorously, with the mean downward attenuation coefficient averaged within PAR range $\bar{K}_{d \text {,PAR }}$ ) resulting from our simulation method. In Figure 2.7 (A) and (B) for WT and Olive strain respectively, it can be seen that there is a reasonable cor- 
relation between experimental and in silico results. Small discrepancies arise for the case of the most diluted cultures, where the attenuation is somewhat underestimated in both strains, though data do not show a clear tendency. Relative error for the attenuation coefficient comparison at this cell concentration is quantified to be around $25 \%$ in WT strain, 30\% in Olive and much lower in the other density cases for both strains. At this OD $\left(0.1 \mathrm{OD}_{750}\right)$, we estimated the acclimation irradiance to be around $90 \%$ of the nominal incident value of $120 \mu \mathrm{mol}$ photons $\cdot \mathrm{m}^{-2} \cdot \mathrm{s}^{-1}$. At this average intensity, expressed as a percentage of the incident irradiance at depth $z=0$, the total attenuation coefficients (in $\mathrm{m}^{-1}$ ) suffer little bit higher variations and particularly started to decline in our laboratory WT strain (data not shown). On the contrary, at higher cell-densities the average irradiance is lower, around $20 \mu \mathrm{mol}$ photons $\cdot \mathrm{m}^{-2} \cdot \mathrm{s}^{-1}$, and in this light environment optical spectra do not vary much at different light intensities and thus the uncertainty of the irradiance level has a minor impact on the light decay slope.

Particularly in the case of Olive cultures it is noteworthy to mention that, as in the case of the WT suspension profiles, the model predicts the attenuation for all $\mathrm{OD}_{750}$ values quite accurately with the exception of the Olive samples at the $\mathrm{OD}_{750}$ value of 2.5 then the corresponding attenuation is overestimated (associated error of $14 \%$ ).

The estimated average irradiance inside the simulated cultures of 0.5 $\mathrm{OD}_{750}$ in Lea-Smith et al. experiment conditions, is $45 \%$ and $56 \%$ for WT and Olive strain, respectively. These are very close to the ones that were hypothesised ( $49 \%$ and $60 \%$ ) to assess the average irradiance in our laboratory conditions (same reactor depth, density and very similar lamp irradiance, 100 instead of $120 \mu \mathrm{mol}$ photons $\cdot \mathrm{m}^{-2} \cdot \mathrm{s}^{-1}$ ), reinforcing the auto-consistency of our algorithmic approach with respect to attenuation.

Finally, in order to better appreciate the general trend for all the densities, experimental and theoretical chl a-specific downward attenuation coefficients $\bar{K}_{d, P A R}^{\star}$ were obtained and plotted together. To do so, the 
experimental data set that was described above and is shown in Figure 2.7 (A) and (B) for each strain was used to estimate the experimental downward attenuation coefficients. More precisely, the irradianceweighted attenuation coefficients were calculated and compared with our results. As displayed in Figure 2.8, the model is able to predict the tendency of the coefficients. It is noteworthy to remark that as the cell-density increases, the average irradiance (and the attenuation coefficient) gradually decreases. Accordingly, if ultra-dense cultures were employed, the mean light intensity would tend to zero. In this hypothetical situation, cells would not have enough energy to sustain biochemical processes and probably long-term adverse effects would appear in metabolism that could impact the optical properties.

\subsubsection{Attenuation profiles for colour light exposure}

Next, we benchmark our modelled attenuation results with the experiments carried out again by Lea-Smith and co-workers, in which cultures were exposed to blue, green, red and also white LED light at a single optical density of $1.0 \mathrm{OD}_{750}$. In this case the irradiance used to measure attenuation was $1,000 \mu \mathrm{mol}$ photons $\cdot \mathrm{m}^{-2} \cdot \mathrm{s}^{-1}$, instead of $2,000 \mu \mathrm{mol}$ photons $\cdot \mathrm{m}^{-2} \cdot \mathrm{s}^{-1}$.

Regarding this data assessment, we were not initially able to properly model the sample points: obtained simulated profiles following the previously described reasoning were only matching experimental data for white light in both strains, and red in the WT strain. In the other cases, the model clearly underestimated the experimental sample values. This modelling mismatch occurred for both strains, and hence, the simulation using one colour series of data was adjusted to check if knowing the average irradiance would be sufficient to correctly predict the light decay in all cases.

This procedure was used with the WT culture blue light essay as depicted in Figure 2.7 (C) by calculating the acclimation irradiance in the growth conditions that would allow one to fit the data. It was found that the acclimation light intensity that delivers satisfactory results is 
$40 \%$ higher than the value that was supposed to exist inside the conical flasks for both strains. Interestingly, only changing this value, the remaining five experiments analysed in this subsection and plotted in Figure 2.7 (C) and Figure 2.7 (D), for WT and Olive mutant respectively, were correctly predicted. One reason to explain this unexpected growth irradiance could be that cultures had been kept in other illumination conditions for some period of time, consequently having adapted and changed their absorption capacity before the attenuation measurement. Moreover, green colour is in many cyanobacterial cultures the one that is less attenuated and this is also the particular case in Synechocystis suspensions. This fact also supports our idea that, for this series of experiments, there are two groups of cultures, each one acclimatised to a different mean irradiance. Indeed, Figure 2.7 (C) displays the attenuation for WT cells illuminated with the four different LED lamps and unexpectedly green attenuation seems to be higher than white one though all cells have been cultivated in the same conditions and thus green should be the least attenuated colour.

Hence, we have adopted the working hypothesis that the assays described in this section correspond to cultures acclimatised to two different light conditions, thus possessing two differentiated "optical footprints" and by assuming this fact, we have been able to precisely estimate light attenuation at different exposure LED light in both strains. Analysing the results in more depth by comparing both strain profiles for a given light colour, it is obvious to realise that blue and green attenuation are quite similar in both strains, around $165 \mathrm{~m}^{-1}$ for blue light, 81 and $95 \mathrm{~m}^{-1}$ for green radiation in Olive and WT strain, respectively, whereas red attenuation in Olive has clearly diminished due to the lack of phycobilisome antennas. For this colour downward attenuation coefficient accounts for $125 \mathrm{~m}^{-1}$ in WT.

In contrast to white light exposure assays, these trials show a purely exponential decay. On the other side, when observing white light attenuation, a two-zone behaviour around a turning point of approximately $1 \%$ (20 $\mu \mathrm{mol}$ photons $\cdot \mathrm{m}^{-2} \cdot \mathrm{s}^{-1}$ ) of incident irradiance is apparent. Below this threshold attenuation diminishes. As already mentioned, white 
light is comprised by different wavelengths and in general green light is the least attenuated. When most light has been absorbed by the medium, only green radiation remains in the PBR and thus a smaller attenuation is expected. This can be better understood from our simulations shown in Figure 2.10 where the initial white light lamp emission spectrum is gradually transformed into a green colour one.

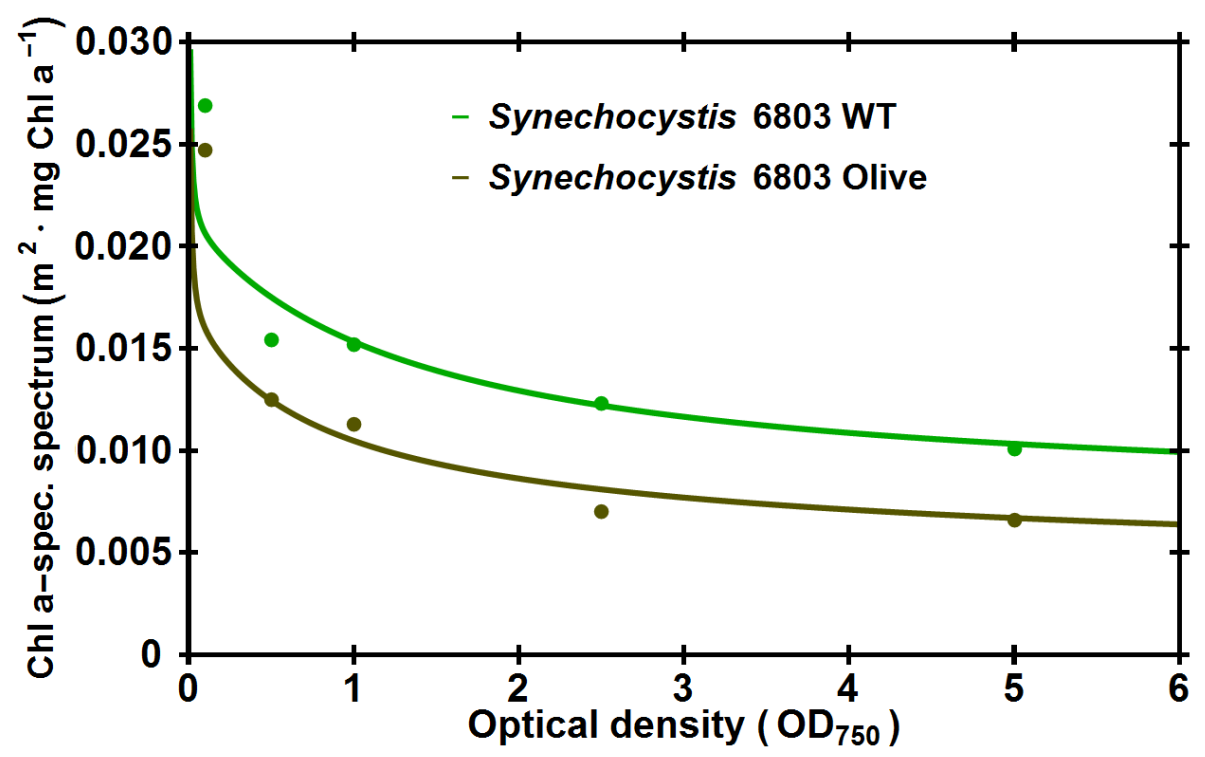

Figure 2.8: Chlorophyll a-specific mean downward attenuation coefficient $\bar{K}_{d, P A R}^{\star}\left(E_{d, a c c}\right)$ comparison for WT and Olive strains (green and brown colour, respectively) between experimental (dots) and modelled values at the given densities. Experimental coefficients were obtained from the white lamp exposure assays at 2,000 umol photons $\cdot \mathrm{m}^{-2} \cdot \mathrm{s}^{-1}$ and calculated as irradiance-weighted attenuation coefficients, whereas the in silico values were directly obtained by dividing by the chlorophyll amount for each optical density.

\subsubsection{Spectrally dependent penetration depth and attenuation}

The previously described results correspond to the integrated attenuation within the PAR range. This type of measurement is a more common and practical way to evaluate irradiance and therefore it is much easier to find information of trials on PAR attenuation in photosynthetic 
microorganisms rather than to describe the spectrally-dependent light attenuation within the cultures. Further, to calculate the light penetration with spectral resolution, we have to solve Beer-Lambert equation for the distance inside the culture at which the irradiance falls to a threshold value, for instance the $10 \%$ of the initial photon flux for each wavelength. This value roughly represents the limit depth at which net cell respiration will occur at the simulated conditions of this subsection.

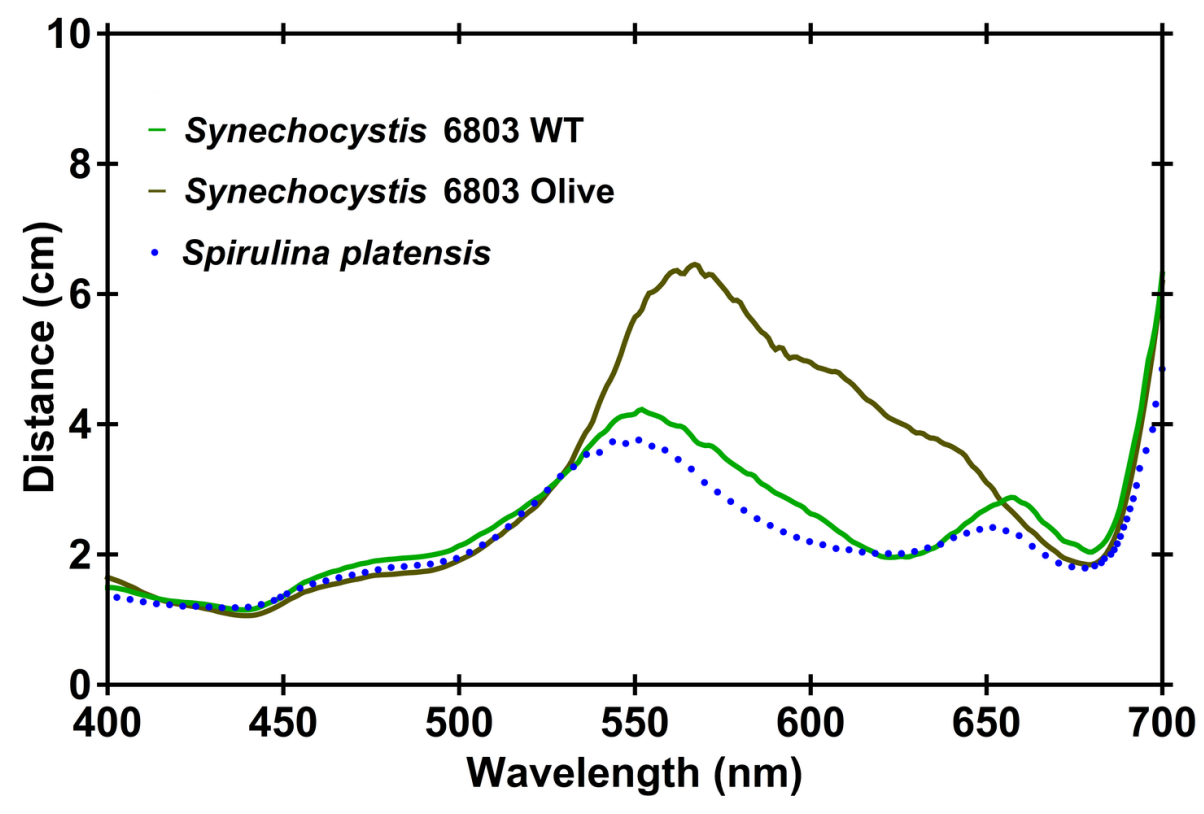

Figure 2.9: Penetration depth at which irradiance drops to $10 \%$ of the initial value at each wavelength within PAR range for WT and Olive strains (green and brown colour, respectively). Simulation conditions correspond to Synechocystis WT cultures grown at typical PBR densities, i.e. $1.0 \mathrm{OD}_{750}$, and acclimatised to a lamp irradiance of 80 $\mu \mathrm{mol}$ photons $\cdot \mathrm{m}^{-2} \cdot \mathrm{s}^{-1}$ of white light. Blue dots correspond to Spirulina platensis depths estimated from experimental attenuation coefficients.

To check that our algorithm also delivers reliable results when purely spectral assessment of light is taken into consideration, an extensive literature review was conducted in order to find relevant contributions with such type of measurements. Unfortunately, we did not find any analogous experiments on Synechocystis and therefore we looked into available attenuation coefficients and penetration profiles with spectral resolution of cyanobacterial species such as Spirulina platensis $M$ - 
2, a species which is very close to Synechocystis. Suspensions of this organism at very high concentrations were examined and penetration depths measured Gitelson et al. (1996). These wavelength-dependent depths were not directly estimated but calculated from experimentally obtained values of $K_{d}(\lambda, z)$, measuring the downward light flux with a radiometer at the surface and at some depth within the cell suspension.

So, we simulated Synechocystis WT cultures at typical PBR densities, i.e. $1.0 \mathrm{OD}_{750}$, and acclimatised to $80 \mu \mathrm{mol}$ photons $\cdot \mathrm{m}^{-2} \cdot \mathrm{s}^{-1}$ lamp irradiance, which approximately corresponds to an incident irradiance of almost $200 \mu \mathrm{mol}$ photons $\cdot \mathrm{m}^{-2} \cdot \mathrm{s}^{-1}$ in a 4 -cm deep PBR. Then we benchmarked our strain penetration profile with the Spirulina one that would arise from cells of the latter organism owning similar spectral attenuation coefficients $\bar{K}_{d}\left(\lambda,\left[z_{1}, z_{2}\right]\right)$. If we assume that attenuation keeps constant at each wavelength, we can qualitatively benchmark both species penetration profiles at equivalent concentrations as these profiles shape is by definition constant (i.e. depths for a light decay to $1 \%$ of incident irradiance are exactly double than the corresponding to $10 \%)$. In other words, we perform a qualitative assessment to validate our results.

In Figure 2.9 we can observe that the indirectly measured Spirulina penetration depths and WT Synechocystis calculated ones practically overlap each other. This shows that the model is also capable of predicting properties that have spectral resolution, such as wavelength dependent light attenuation. Moreover, as seen in the plot, Olive penetration depths are similar to WT ones within the whole PAR range with the remarkable exception of the red band, due to the previously mentioned phycobilisome absence. In this spectral region, Olive cultures allow an additional two centimetres of light penetration in comparison with WT in the given conditions.

Finally, we did calculate another optical property: the spectral photon flux density within the cultures taking into account the four lamps assessed in this contribution. Simulated environment in the PBR was hypothesised for an $80 \mu \mathrm{mol}$ photons $\cdot \mathrm{m}^{-2} \cdot \mathrm{s}^{-1}$ mean acclimation inten- 
sity, at an incident exposure irradiance of $1,000 \mu \mathrm{mol}$ photons $\cdot \mathrm{m}^{-2} \cdot \mathrm{s}^{-1}$ in a 4-cm deep PBR and with a suspension $\mathrm{OD}_{750}$ value of 1.0. The result is depicted in Figure 2.10 at 0,1 and $2 \mathrm{~cm}$ depth within the PBR.

The most interesting feature of such a spectral description of light is to gather information on the remaining irradiance at target wavelengths that can promote specific photosynthetic processes at a deeper depth. The differentiated effect of attenuation on specific wavelengths can be better appreciated in the white light example as green band photons are much less attenuated and they are the predominant colour at deeper distances.

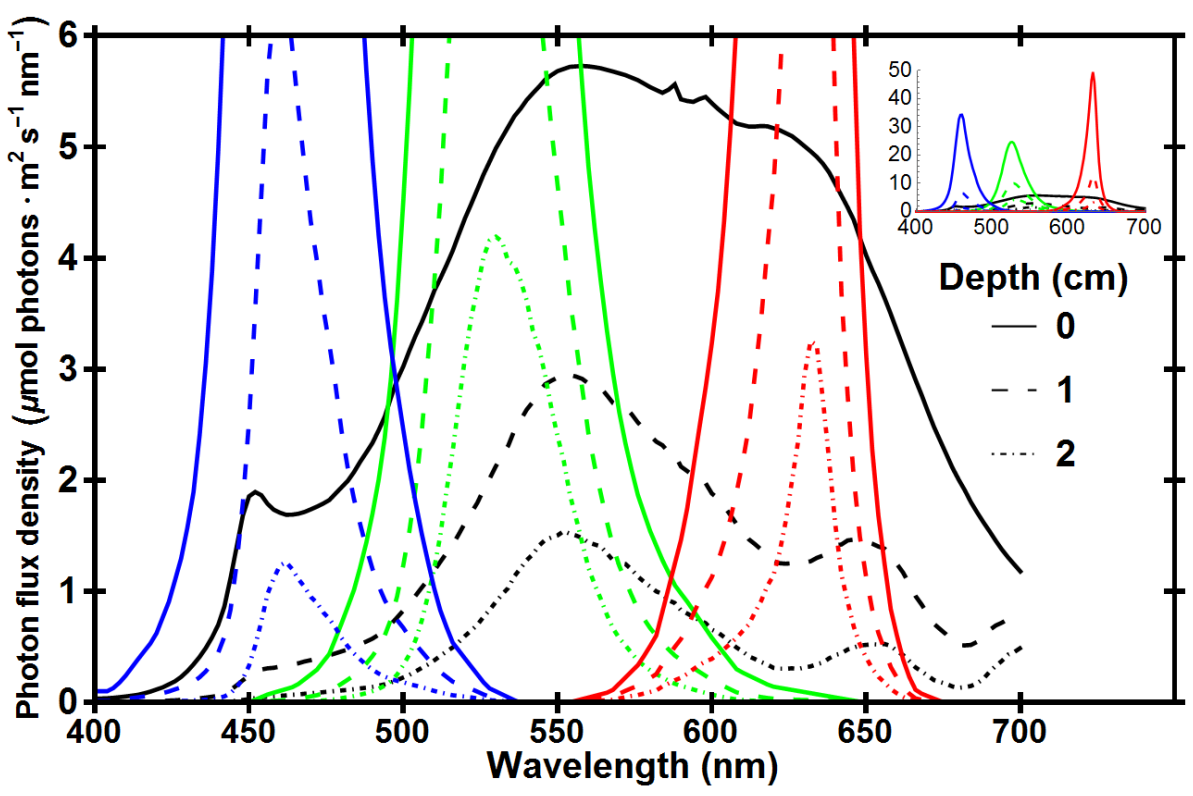

Figure 2.10: Modelled spectral photon flux densities $\rho E_{d}(\lambda, z)$ within simulated Synechocystis WT strain cultures are depicted, where cells are adapted to white light of $80 \mu \mathrm{mol}$ photons $\cdot \mathrm{m}^{-2} \cdot \mathrm{s}^{-1}$ but momentarily exposed to 1,000 $\mu$ mol photons . $m^{-2} \cdot s^{-1}$ of different colour LED lamps (white, blue, green or red light) at an $\mathrm{OD}_{750}$ concentration of 1.0. Remaining photon flux densities at 0, 1 (dashed) and $2 \mathrm{~cm}$ (dotted) are shown. Inset plot shows whole graphs with the same units in both axes. 


\subsubsection{Attenuation coefficient formula in Synechocystis cultures}

Once the algorithm has been validated, simulations can be performed to estimate the PAR downward attenuation for both strain cultures given the incident irradiance, the length of the PBR and a constant cell density inside the reactor. As a representative example, attenuation coefficients for the studied range of cell densities and light intensities inside a PBR with a depth of $4 \mathrm{~cm}$ are shown in Figure 2.11. It can be observed that the slope of attenuation coefficients is higher at lower chlorophyll concentration values. In these conditions, average irradiance inside the suspension drops very quickly and as a result, the chlorophyll-specific attenuation $\bar{K}_{d, \mathrm{PAR}}^{\star}\left(E_{d, a c c}\right)$ does too (Figure 2.6). Above concentration values of $10,000 \mathrm{mg} \mathrm{chl} \mathrm{a} \cdot \mathrm{m}^{-3}$ average irradiance is kept low, practically constant. Similarly the chlorophyll-specific attenuation coefficient stays low, too. In this way, from a given chlorophyll amount, the resulting attenuation coefficient for downward irradiance increases linearly.

Finally, as a practical outcome of our investigation, our procedure delivers a simplified general estimation of PAR attenuation in different acclimatisation conditions for Synechocystis suspension within flat-type one side illuminated PBRs. For this purpose, the obtained data were correlated by an empirical equation:

$$
\begin{gathered}
\bar{K}_{d, \mathrm{PAR}, W T}=\frac{17.9+0.0178 \cdot \epsilon}{200+1049 \cdot \delta} \cdot \rho^{0.8} \\
\bar{K}_{d, \mathrm{PAR}, \text { Olive }}=\frac{13.7+0.0234 \cdot \epsilon}{216+1938 \cdot \delta} \cdot \rho^{0.8}
\end{gathered}
$$

where $\epsilon$ represents the incident irradiance emitted by the employed lamp ( $\mu \mathrm{mol}$ photons $\cdot \mathrm{m}^{-2} \cdot \mathrm{s}^{-1}$ ), $\delta$ stands for the depth of the photobioreactor $(\mathrm{m})$ and $\rho$ is the cell density expressed as the concentration of chlorophyll a in the suspension $\left(\mathrm{mg} \mathrm{chl} \mathrm{a} \cdot \mathrm{m}^{-3}\right)$. The estimation of the downward attenuation coefficient, expressed in $\mathrm{m}^{-1}$, is valid within the 
analysed range of average intensities, which accounts for roughly 10$100 \mu \mathrm{mol}$ photons $\cdot \mathrm{m}^{-2} \cdot \mathrm{s}^{-1}$. For typical PBR depths and the already assessed cell-densities, this operation interval corresponds to 20-150 $\mu \mathrm{mol}$ photons $\cdot \mathrm{m}^{-2} \cdot \mathrm{s}^{-1}$ incident irradiance. The other variable ranges are $0.01-0.10 \mathrm{~m}$ for the PBR depth and $0-25,000 \mathrm{mg} \mathrm{chl} \mathrm{a} \cdot \mathrm{m}^{-3}$ for the chl a concentration. Additionally, our IOPs spectra were obtained under a cool white light LED so the attenuation coefficients estimated here will likely not be the same when a light source with dissimilar spectral characteristics is employed.

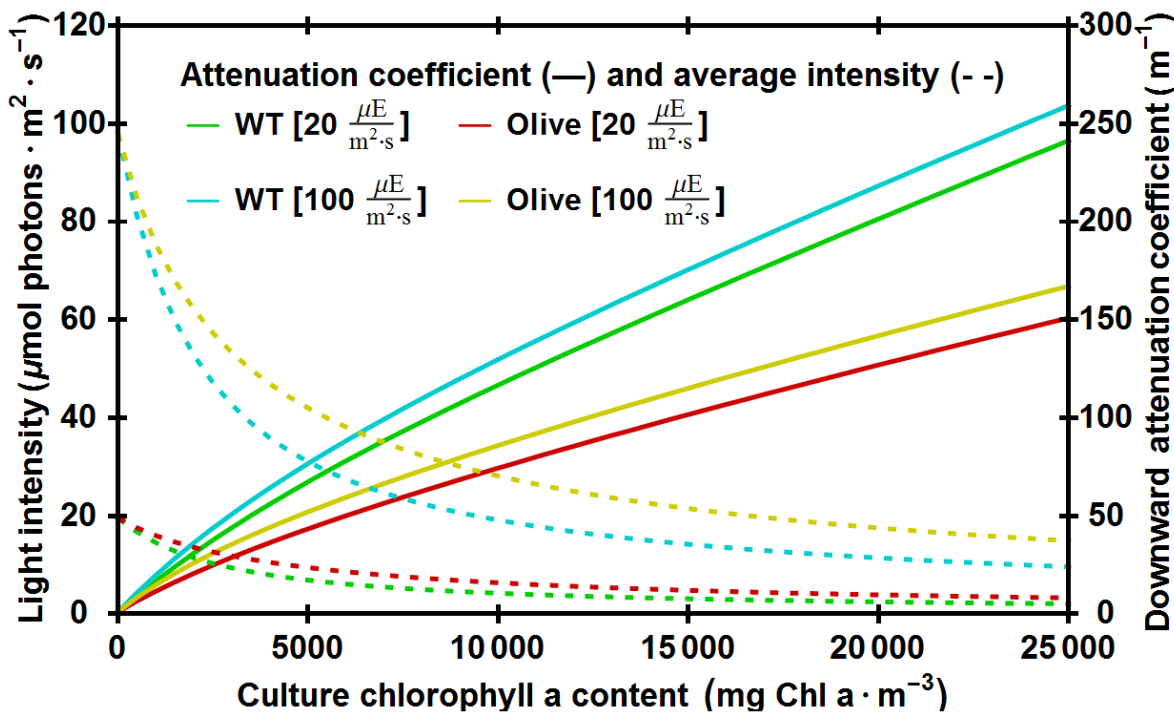

Figure 2.11: Modelled mean downward attenuation coefficient within PAR range $\bar{K}_{d, P A R}\left(E_{d, a c c}\right)$ for WT cultures exposed to lamp intensities of 20 and 100 $\mu \mathrm{mol}$ photons $\cdot \mathrm{m}^{-2} \cdot \mathrm{s}^{-1}$ in a 4-cm depth PBR at constant cell-densities up to 25,000 $\mathrm{mg} \mathrm{chl} \mathrm{a} \cdot \mathrm{m}^{-3}$ are depicted (right vertical axis). Additionally, resulting average irradiance $E_{d, \text { acc }}$ is also plotted for such suspensions (dotted, left vertical axis). Green and blue curves stand for WT cultures grown at 20 and $100 \mu \mathrm{mol} \mathrm{photons} \cdot \mathrm{m}^{-2} \cdot \mathrm{s}^{-1}$ incident radiation and similarly red and yellow curves represent Olive cultures cultivated at 20 and $100 \mu \mathrm{mol}$ photons $\cdot \mathrm{m}^{-2} \cdot \mathrm{s}^{-1}$ incident radiation. 


\subsection{Conclusions and future work}

In this work, a new model to estimate downward light attenuation has been presented. The described methodology makes use of a semiempirical correlation that was developed for marine biology applications. This, together with some simplifying assumptions of homogeneity, acclimation response of the cells and linearity of the Inherent Optical Properties, allows one to make predictions about the average field inside the PBR and the corresponding attenuation light profile. The proposed mathematical algorithm is based on the solution of a selfconsistency problem: the average irradiance depends on the downward attenuation coefficient and vice versa. Moreover, it can be applied to any type of PBR geometry, lamp arrangement and spectra, although in our work we have derived concrete expressions for the case of a flattype PBR illuminated on just one side.

To check the validity of this approach, a combined analysis of experiments performed by the authors of this work together with the data obtained by Lea-Smith and co-workers was carried out on the same organisms, namely WT and Olive strains of Synechocystis.

Despite the different assumptions, we have benchmarked our predictions with experimental data to show that the model is able to reasonably predict light attenuation for both strains at various OD values and different colour LED light with a small support of additional assumptions. Thus, we conclude that it is possible to predict the light field inside PBRs operating under a broad range of conditions with a reduced set of previously-measured Inherent Optical Properties of the organism of interest. Moreover, knowing the exact acclimatisation intensity would allow a better prediction of the real attenuation profiles.

Our methodology opens further possibilities, e.g. to evaluate other illumination conditions and benchmark photosynthetic organisms, assessing possible improvements on the cultivation conditions and the PBR set-up. A further research line should cope with photo-adaptation and photo-inhibition dynamics, considering optical spectra changes upon 
radiation variations. In this regard, to leverage in silico absorption coefficient estimations in terms of light quality and quantity changes, further information on pigment concentration is desired. Additionally, this model can be coupled to others describing the production of oxygen or other compounds, allowing an improvement of their prediction capacity.

In summary, it is getting more common to study the light impact on photosynthesis, not just for optimising large-scale photobioreactor operation but also to better understand the underlying mechanisms that trigger optically-dependent processes that control photosynthesis, and therefore metabolism, indirectly. In this regard, our approach aims to be the first step towards a more integrative modelling of optical properties inside PBR cultures and to better understand the challenge of describing the effect of light on photosynthetic microorganisms.

\subsection{Acknowledgements}

This project has received funding from the European Union's Seventh Programme for research, technological development and demonstration under grant agreement No 308518 CyanoFactory, to Javier Urchue guía's and Matthias Rögner's respective research groups and from the grant Contratos Predoctorales FPI 2013 of the Universitat Politècnica de València to the first one. We would also like to thank David LeaSmith and Dariusz Stramski for their fruitful and selfless contribution. We kindly acknowledge the experimental support of Saori Fuse for the cultivation of cyanobacteria. 



\section{3}

\section{Estimation of light field}

inside photosynthetic

\section{microorganism cultures}

\section{through Mittag-Leffler}

\section{functions}

Title: Estimation of the light field inside photosynthetic microorganism cultures through Mittag-Leffler functions at depleted light conditions

Reference: Journal of Quantitative Spectroscopy \& Radiative Transfer 204 (2018) 23-26

Authors: David Fuente ${ }^{a, *}$, Carlos Lizama ${ }^{b}$, Javier F. Urchueguía ${ }^{a}, \mathrm{~J}$. Alberto Conejero ${ }^{c}$

${ }^{a}$ Instituto de Aplicaciones de las Tecnologías de la Información y de las Comunicaciones Avanzadas, Universitat Politècnica de València, València, Spain

${ }^{b}$ Universidad de Santiago de Chile, Facultad de Ciencia, Departamento de Matemática y Ciencia de la Computación, Santiago de Chile, Chile 
${ }^{c}$ Instituto Universitario de Matemática Pura y Aplicada, Universitat Politècnica de València, Valencia, Spain

* Corresponding author: dafueher@upv.es

\section{Abstract}

Light attenuation within suspensions of photosynthetic microorganisms has been widely described by the Lambert-Beer equation. However, at depths where most of the light has been absorbed by the cells, light decay deviates from the exponential behaviour and shows a lower attenuation than the corresponding from the purely exponential fall. This discrepancy can be modelled through the Mittag-Leffler function, extending Lamber-Beer law via a tuning parameter $\alpha$ that take into account the attenuation process.

In this work, we introduce a fractional Lambert-Beer law to estimate light attenuation within cultures of model organism Synechocystis sp. PCC 6803 Montagud et al. (2011). Indeed, we benchmark measured light field inside cultures of two different Synechocystis strains, namely the wild-type and the Olive antenna mutant strain at five different cell densities, with our in silico results. The Mittag-Leffler hyper-parameter $\alpha$ that best fits the data is 0.995 , close to the exponential case. We show that by applying the fractional Lambert-Beer law for describing light attenuation, we are able to properly model light decay in photosynthetic microorganisms suspensions.

Keywords: Light field, attenuation, Lambert-Beer law, Mittag-Leffler, modelling, Synechocystis

\subsection{Introduction}

Photosynthetic microorganisms are capable of absorbing light by means of a wide variety of photo-active pigments, whose active molecules can 
get excited at given wavelengths. Photosynthetic pigments cover the so called Photosynthetically Active Radiation (PAR), comprising the wavelength range of 400 to $700 \mathrm{~nm}$, which practically overlaps the visible spectrum for human beings.

Light decay in photosynthetic cultures is due to absorption and scattering phenonema Mishchenko (2014b), can be described using inherent optical properties of the suspension Preisendorfer (1961) and by solving a self-consistency problem between average irradiance inside the whole volume and the average PAR downward attenuation coefficient, as both magnitudes depend on each other. In this regard, a modelling approach was recently proposed Fuente et al. (2017), where the remaining light given as a PAR integrated irradiance value at different depths from the emission lamp within Synechocystis sp. PCC 6803 (hereafter referred to as Synechocystis) cultures was in silico estimated.

However, the model employs the Bouguer law, postulated in Bouguer's Essai d'Optique in 1729 Mishchenko (2014a) and commonly known in Optics as Lambert-Beer law, for estimating remaining irradiance up on depth and hence it fails in the evaluation of very low light intensities. Despite light usually follows an exponential decay that can be described by the Lambert-Beer law, it is known that at low illumination conditions, below the so called euphotic zone, light attenuation deviates from a purely exponential behaviour inside biological cultures. As a rule of thumb, the exponential case is valid within the interval comprised between that layer of water from the surface down to the depth at which downward irradiance is $1 \%$ of that at the depth $z=0$ Kirk (2003).

This has been reported in several works on oceanic environments Kirk $(1977,1994)$ but also in photosynthetic microorganism cultures grown in artificial conditions Lea-Smith et al. (2014). To overcome the poor attenuation prediction capabilities of current models near or within the euphotic zone, the algorithms can be modified to extend exponential attenuation to a more complex representation by means of Mittag-Leffler (ML) functions. These are solutions of fractional order integral and 
fractional order differential equations and have been successfully introduced in several applied areas for studying different problems such as rheology Schiessel et al. (1995), diffusion in porous solids Zaman et al. (2016), superdiffusive transport and Lévy motion Metzler and Klafter (2000), protein ligand rebinding Glöckle and Nonnenmacher (1995), electrical networks Calık et al. (2016) and, in general, they are useful to study relaxation phenomena in complex physical systems BerberanSantos (2005); Haubold et al. (2011) and in probability theory Mainardi et al. (2005). Moreover, it is remarkable that ordinary and generalised Mittag-Leffler functions interpolate between a purely exponential law and a power-like behaviour of phenomena governed by ordinary kinetic equations and their fractional counterparts Hilfer (2000); Lang (1999); Saxena et al. (2002).

Regarding optical applications with such class of mathematical functions, in Preda (2016) a ML function for the modulation of a Gaussian beam was employed and the splitting and accelerating properties of the obtained Mittag-Leffler-Gaussian beam described. Recently, the light attenuation modelling through a generalisation of the exponential function via ML functions was considered Casasanta et al. (2012). That generalisation of the Lambert-Beer rises the accuracy of the predictions and explains possible deviations from the exponential extinction law in spatially correlated media. This model was later improved by introducing a new formal description that included Wright type functions to express light transmission probability in random media Tramontana et al. (2013). Estimations of the deviation from the exponential law were also exposed. In this way, multi-order fractional operators (integral and differential) in the unit disk have been also used for extending the Lambert-Beer equation Ibrahim and Ozel (2016).

Nevertheless, in none of these works experimental data were assessed. In summary, we have considered the fractional generalisation of the Lambert-Beer law and modelled light attenuation within cultures of model organism Synechocystis sp. PCC 6803. An interesting outcome of this research is that all experimental measurements are properly modelled by just fitting the hyper-parameter $\alpha$ of the ML functions. Indeed, 
just one value is needed for both studied Synechocystis strains, namely the wild-type and the antenna mutant strain called Olive at all, i.e. five different, cell densities. These results back the use of fractional calculus for describing light attenuation in photobioreactors (PBR).

\subsection{Methodology}

Mittag-Leffler functions, introduced by the homonym Swedish mathematician Gösta Mittag-Leffler, belong to a family of special functions characterized by two complex parameters $\alpha$ and $\beta$. When the real part of $\alpha$ is strictly positive, they can be defined by means of the following convergent series:

$$
E_{\alpha}(z)=\sum_{k=0}^{\infty} \frac{z^{k}}{\Gamma(1+\alpha k)}, \quad \alpha \in \mathbb{C}, \quad \Re(\alpha)>0, \quad z \in \mathbb{C},
$$

or in its more general form by

$$
E_{\alpha, \beta}(z)=\sum_{k=0}^{\infty} \frac{z^{k}}{\Gamma(\beta+\alpha k)}, \quad \alpha, \beta \in \mathbb{C}, \quad \Re(\alpha)>0, \quad \Re(\beta)>0, \quad z \in \mathbb{C}
$$

The positivity of the real parts of alpha and $\beta$ yields that they become entire functions. Here, $\Gamma(\cdot)$ stands for the Gamma function of Euler, that generalizes the factorial, since $\Gamma(n):=(n-1)$ ! for every natural number $n \in \mathbb{N}$. For every $z \in \mathbb{C}$ with $\Re(z)>0$, this function can be defined by the following convergent improper integral

$$
\Gamma(z):=\int_{0}^{\infty} t^{z-1} e^{-t} d t
$$

ML functions generalize the exponential functions. In particular, if $\alpha=$ $\beta=1$, we get the series of the exponential function. They arise as solutions of the following abstract Cauchy problem

$$
\left\{\begin{array}{l}
D_{x}^{\alpha} f(x)=-\lambda f(x), \quad x>0 \\
f(0) \in \mathbb{R} \text { given. }
\end{array}\right.
$$


where $D_{x}^{\alpha}$ stands for the Caputo fractional derivative of real order $\alpha$ respect to $x$. An easy way to understand what is a fractional derivative, one can think that $D_{x}^{1 / 2}$ will be the operator whose square coincides with the usual derivative, i.e. $\left(D_{x}^{1 / 2}\right)^{2}(f)=f_{x}$. There are several ways of introducing the fractional derivative, such as Riemann-Liouville or Caputo fractional derivatives. See also Atangana and Baleanu (2016) for the recent Atangana-Baleanu derivatives. In contrast to the case of Riemann-Liouville's, Caputo fractional derivatives permits to solve differential equations without having fractional initial conditions. Further information on fractional calculus can be found in Podlubny (1998); Ortigueira (2011); Baleanu et al. (2012).

\subsection{Fractional calculus in optics}

Lambert-Beer law states that the evolution of the downward irradiance at the depth $x$ at any wavelength $\lambda$, namely $I_{d}(x, \lambda)$, can be described by

$$
\left\{\begin{array}{l}
\frac{d}{d x} I_{d}(\lambda, x)=\bar{K}_{d}(\lambda) I_{d}(\lambda, x), \quad x>0 \\
I_{d}(\lambda, 0) \in \mathbb{R}^{+}
\end{array}\right.
$$

being $\bar{K}_{d}(\lambda)$ the corresponding spectrally related downward irradiance attenuation coefficient, which can in turn be obtained by using inherent optical properties of each organism Kirk (1984), i. e. measuring chlorophyll-specific absorption and scattering spectra at different mean acclimation irradiance values, when we deal with photosynthetic microorganism cultures.

Furthermore, in Kostinski (2001) deduction of the Lambert-Beer law was explained by means of stochastic approach and linked with Poisson statistics. Later a generalised Lambert-Beer law through fractional calculus was postulated Casasanta et al. (2012). These results could have been first interpreted as local analysis Borovoi (2002), however it was shown that the domain of validity is broader Kostinski (2002). In fact, the same result can be obtained when replacing the ordinary 
derivative by the fractional derivative in Beer-Lambert law (3.5), that is

$$
\left\{\begin{array}{l}
\left(\frac{d}{d x}\right)^{\alpha} I_{d}(\lambda, x)=\bar{K}_{d}(\lambda) I_{d}(\lambda, x), \quad x>0 \\
I_{d}(\lambda, 0) \in \mathbb{R}^{+} \text {given, }
\end{array}\right.
$$

whose solution is given by

$$
I_{d}(\lambda, x)=E_{\alpha}\left(\bar{K}_{d}(\lambda) x^{\alpha}\right) I_{d}(\lambda, 0)
$$

In this way, a nonlocal analysis is introduced in the Lambert-Beer law, incorporating a tuning parameter $\alpha>0$ that represents the memory in $x$ of the model, which is absent in Equation (3.5). Observe that for mathematical consistence we must restrict the value of $\alpha$ between 0 and 1 , due to the number $(=1)$ of the initial conditions given in (3.5). In our approach, we have used expression (3.6) to properly model light attenuation in biological suspensions. To do so, Synechocystis experimental attenuation profiles for different light sources have been estimated by fitting the parameter $\alpha$ of the ML function. It is critical to note that Mittag-Leffler functions do own the necessary properties to correctly predict remaining irradiance within the biological culture as they display power-law decay at further distances but alternatively coincide with the exponential function at closer ones. Though one could expect a two parameters function perform better than a single one, yet other two parameters functions such as the Gamma family do not fulfil the mentioned requirements and hence fail to satisfactorily model the data. This fact together with the mathematical justification of the generalised Lambert-Beer law through Mittag-Leffler functions Casasanta et al. (2012), supports ML functions as the right candidate for modelling light fields within photosynthetic microorganism cultures and equivalent physical systems.

\subsubsection{Data for modelling assessment}

The data set to be benchmarked with computational results corresponds to Synechocystis cultures exposed to light kept inside a cylindrical ves- 
sel. This container has radiometric sensors every $11 \mathrm{~mm}$ to measure PAR downward irradiance within the biological suspension. In this regard, the Synechocystis attenuation data set Lea-Smith et al. (2014) is composed of light intensity values at increasing depths for the strains here studied (wild-type and Olive) plus two extra antenna mutants (not assessed in this manuscript as we do not own inherent optical properties of those strains), all of them exposed to white LED light at 5 different cell-densities. Such concentrations are given as optical density values $\left(\mathrm{OD}_{750}\right)$ at $750 \mathrm{~nm}$, namely these $\mathrm{OD}_{750}$ values correspond to $0.1,0.5,1.0,2.5$ and 5.0 units.

Moreover, in order to benchmark modelling predictions with the described experimental data, the PAR downward attenuation coefficient $\bar{K}_{d}(P A R)$ needs to be first calculated from the spectrally dependent one $\bar{K}_{d}(\lambda)$. To this purpose, measured inherent optical properties altogether with the depth of the photobioreactor, the cell-density and also the intensity and emission spectrum of the light source have to be taken into account. Following this strategy, classical Lambert-Beer law predictions are available as described in a previous modelling work Fuente et al. (2017). In the present manuscript, such modelling framework has been upgraded with Mittag-Leffler functions to improve its predictability capacity.

\subsection{Results}

In Figure 3.1, the light field as PAR irradiance value vs. the distance to the illumination source in wild-type (WT) cultures is plotted. It can be seen that in WT for optical densities equal or greater than 1.0, the light field starts to deviate from the pure exponential case, whereas in Olive this happens for optical densities of at least 2.5 (Figure 3.2). The lowest measured light intensity from the original source within the WT cultures was $1.24 \mu \mathrm{mol}$ photons $\cdot \mathrm{m}^{-2} \cdot \mathrm{s}^{-1}$ at $3.3 \mathrm{~mm}$, whereas for Olive cultures it was 1.60 at $4.4 \mathrm{~mm}$ (both at the most concentrated celldensity). For these points, light attenuation is far from Lambert-Beer 
law prediction (a line in the semi-logarithmic plot). Indeed, the obtained values for exponential decay, $0.1 \mu \mathrm{mol}$ photons $\cdot \mathrm{m}^{-2} \cdot \mathrm{s}^{-1}$ in WT strain and $0.3 \mu \mathrm{mol}$ photons $\cdot \mathrm{m}^{-2} \cdot \mathrm{s}^{-1}$ in Olive mutant are $8.1 \%$ and $19 \%$, of the respective experimental values in each strain. This leads to the conclusion that in culture regions where practically all the light has been captured by the cells, the Lambert-Beer law does not satisfactorily represent the remaining light field, at least in terms of PAR range integrated values (dashed lines). In fact, this happens in both strains below the euphotic zone, which in our case roughly corresponds to data below $20 \mu \mathrm{mol}$ photons $\cdot \mathrm{m}^{-2} \cdot \mathrm{s}^{-1}$.

Alternatively, Mittag-Leffler model (solid lines) is able to correctly predict the remaining downward radiance in both strains at any cell density by fitting the ML $\alpha$ parameter to 0.995 .

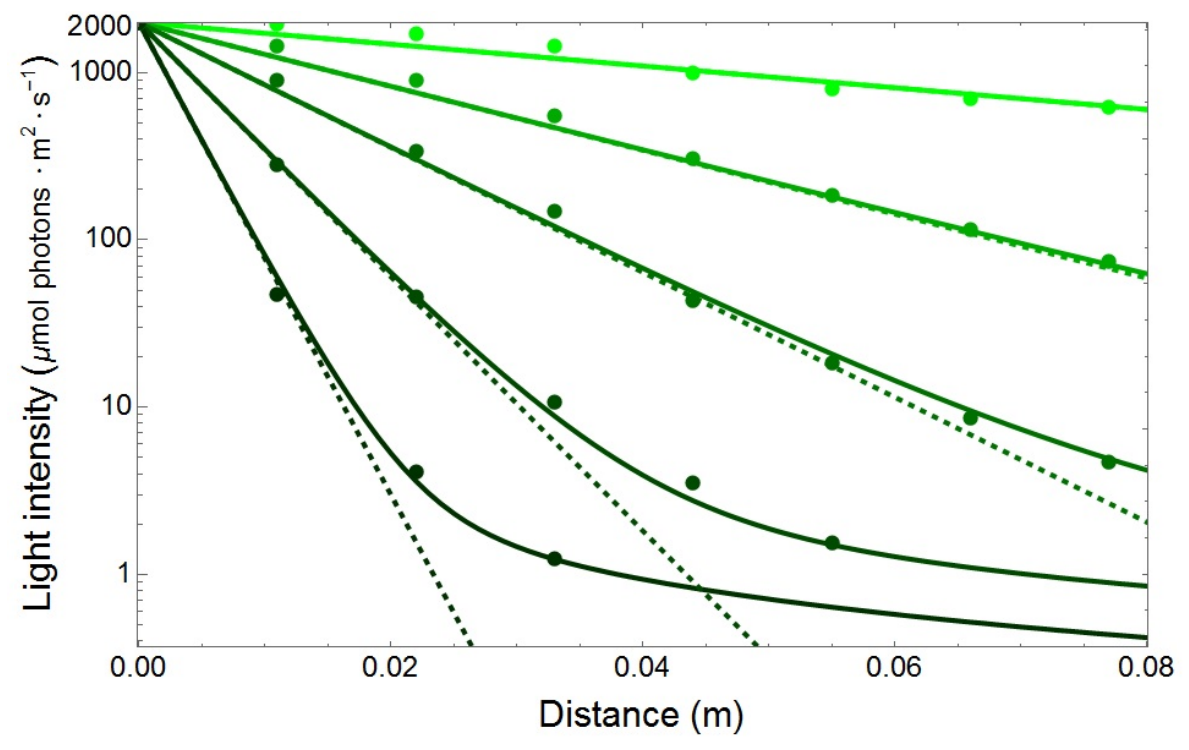

Figure 3.1: Light attenuation profiles of Synechocystis WT strain cultures exposed to $2000 \mu$ mol photons $\cdot \mathrm{m}^{-2} \cdot \mathrm{s}^{-1}$ of white LED light at five different $\mathrm{OD}_{750}$ concentrations $(0.1,0.5,1.0,2.5$ and 5.0) are depicted. Dots are the original source samples and lines the modelling outcome (dashed lines Lambert-Beer law, solid lines Mittag-Leffler function). Darker colours correspond to denser suspensions.

It is also remarkable that for the case of cultures exposed to a lamp spectrum with a very narrow emission band, the attenuation is purely ex- 


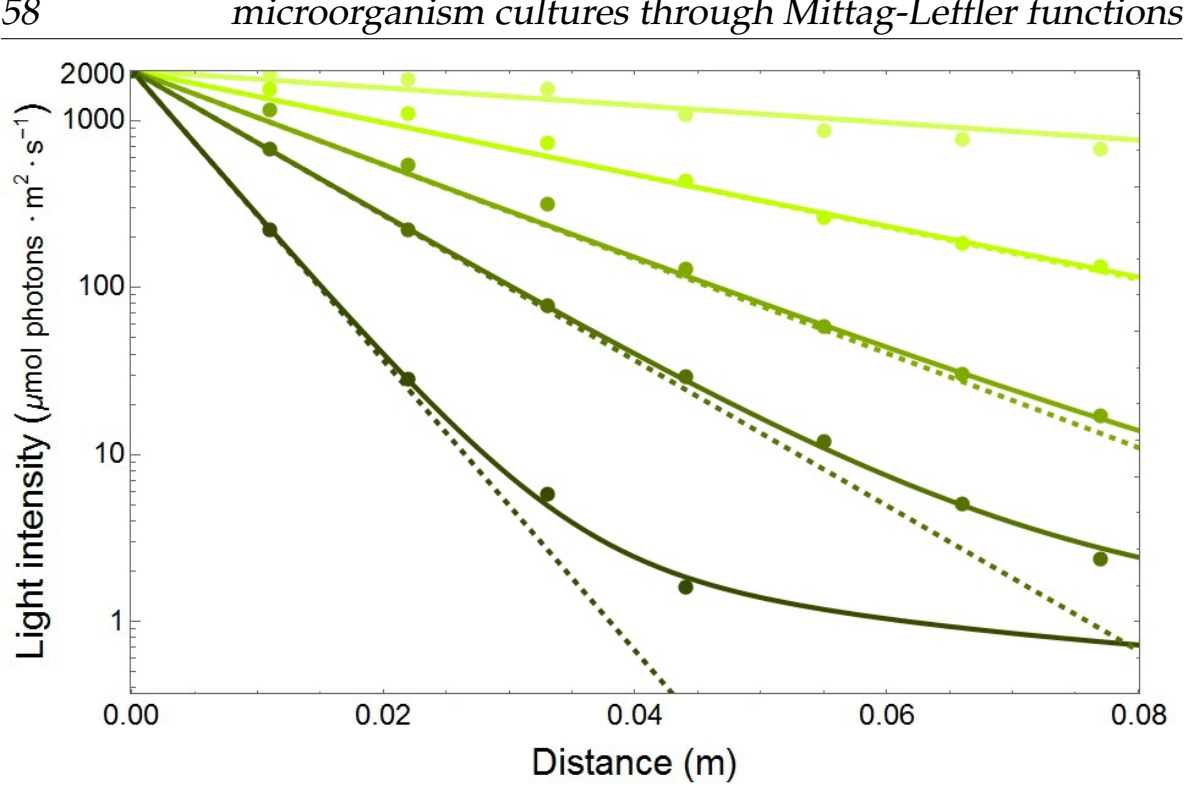

Figure 3.2: Light attenuation profiles of Synechocystis Olive strain cultures exposed to $2000 \mu$ mol photons $\cdot m^{-2} \cdot s^{-1}$ of white LED light at five different $\mathrm{OD}_{750}$ concentrations $(0.1,0.5,1.0,2.5$ and 5.0$)$ are depicted. Dots are the original source samples and lines the modelling outcome (dashed lines Lambert-Beer law, solid lines MittagLeffler function). Darker colours correspond to denser suspensions.

ponential in the measured conditions (data not shown Lea-Smith et al. (2014)). This is due to the fact that monochromatic light is taken up by cells at a constant rate given by the attenuation coefficient value at the peak wavelength of the LED emission spectrum (Gaussian-shaped), whereas in the case of white LED light exposure, attenuation varies gradually: cells mainly absorb light at wavelengths close to the peaks corresponding to the pigments present in them. This selective absorption leads to irradiance diminution of the primarily absorbed wavelengths while the less absorbed ones contribute to the remaining light field at deeper distances. In the case of Synechocystis cells, likewise many cyanobacteria which do not possess any photo-active pigment capable of capturing green radiation, the green components of the spectrum are less absorbed, thus light gradually changes from white at the surface to a greener colour at the furthest depth. This leads to a progressively lower attenuation, as cells can hardly capture green light, which 
explains why a purely exponential decay is not valid in such selectively absorbing media.

\subsection{Conclusions}

In this work we propose a new scheme to model light attenuation within photosynthetic cultures based on the use of Mittag-Leffler functions. Our scheme is able to substantially improve the estimation of how light intensity decreases at culture depths near or within the euphotic zone, where intensity has already decreased substantially and its spectrum has considerably changed due to selective absorption in the medium. Remarkably, the value of the fitting parameter $\alpha=0.995$ that allows for a proper description of light attenuation within the cultures is exactly the same for both experimental sets corresponding to two different Synechocystis strains. This is not a surprising outcome as both strains have almost the same size, display similar pigment concentration and internal arrangement, except for the missing phycocyanin chromophore in the mutant strain. In view of our results, it may be hypothesised that properly tuned Mittag-Leffler functions are able to represent the long range effects due to selective absorption phenomena in photosynthetic cultures. Indeed, this is the first work where experimental data corresponding to a system with a slower-than-exponential light decay have been benchmarked with in silico results. By updating the previous exponential-type published model Fuente et al. (2017) with MittagLeffler functions, accurate results are obtained. In fact, this framework generalisation allows the estimation of the light field at any cell density and optical depth inside a PBR for photosynthetic microorganism cultures acclimated to a given irradiance.

As a further work it would be interesting to test our scheme with other cyanobacterial or micro-algal species and to explain, via an analytic estimation, the value of the hyper-parameter taking into account PBR, light, media, and culture characteristics. Furthermore it would be useful to work with a model that can represent the behaviour of the frac- 

microorganism cultures through Mittag-Leffler functions

tional Lambert-Beer law in the case that the parameter varies between 1 and 2 in a different biological phenomenon. This corresponds to the situation where there are two initial conditions instead of only one as in the classical model (3.5).

\subsection{Acknowledgements}

This project has received funding from the European Union's Seventh Programme for research, technological development and demonstration under grant agreement No 308518 CyanoFactory. David Fuente is supported by grant Contratos Predoctorales FPI 2013 of the Universitat Politècnica de València. Carlos Lizama is supported by Programa de Apoyo a la Investigación y Desarrollo (PAID-02-15) de la Universitat Politècnica de València and CONICYT - PIA - Anillo ACT1416. 


\section{4}

\section{Experimental characterisation of Synechocystis sp. PCC 6803 cultures productivity up on light conditions}

Title: Experimental characterisation of Synechocystis sp. PCC 6803 cultures productivity up on light conditions

Authors: David Fuente ${ }^{a, *}$, Cristina Loureiro $^{b}$, Javier F. Urchueguía ${ }^{a}$

${ }^{a}$ Instituto de Aplicaciones de las Tecnologías de la Información y de las Comunicaciones Avanzadas, Universitat Politècnica de València, València, Spain

${ }^{b}$ Departamento de Física Aplicada, Universitat Politècnica de València, València, Spain

* Corresponding author: dafueher@upv.es 


\section{Abstract}

In this work, we have performed a characterisation of Synechocystis sp. PCC 6803 glucose tolerant strain cultures regarding optical properties, growth, carbon uptake, pigment composition and productivity, among other features, at different optical and cell-density conditions to gain insight into the effect of light and suspension dilution. For a rigorous analysis, average light intensities are first computed to link any culture property to mean light field conditions. Under a red illumination of $110 / 330 \mu \mathrm{mol}$ photons $\cdot \mathrm{m}^{-2} \cdot \mathrm{s}^{-1}$ switch set-up at very diluted conditions $\left(\mathrm{OD}_{720}\right.$ of 0.1$)$, Synechocystis cultures exhibit a short doubling time of roughly 4.6 hours. This is due to the fact that denser cultures exposed to higher light intensities have to cope with very low and high radiation in quick periods of time, while diluted suspensions experience a rather homogeneous light field. In first scenario, photosynthesis has to cope with extreme and irregular light fluctuations, hindering a proper photosynthesis regulation and promoting clums in the suspension due to light stress. Intermittent light was also applied to benchmark results with continuous irradiance, but for our range of frequency switch, continuous light outperforms intermittent one. Among the gathered data, we estimated a photosystem ratio of 5.5 at low intensities, while the maximal and minimal chlorophyll per cell is 100 and $40 \mathrm{fg} \mathrm{chl} \mathrm{a} \cdot \mathrm{cell}^{-1}$.

We also utilise and modify a previous mathematical model to benchmark experimental $\mathrm{O}_{2}$ evolution results with the in silico estimation. We assessed the capacities and limitations of the model: we predict that gross photosynthesis rate is $320 \mu \mathrm{mol}$ photons $\cdot \mathrm{m}^{-2} \cdot \mathrm{s}^{-1}$, and that photorespiration rates overtake dark respiration above a mean intensity of $400 \mu \mathrm{mol}$ photons $\cdot \mathrm{m}^{-2} \cdot \mathrm{s}^{-1}$, rising up to levels that are three times the dark respiration under very high intensities. Besides, a maximum volumetric culture performance of around $600 \mu \mathrm{mol} \mathrm{O} 2 \cdot \mathrm{L}^{-1} \cdot \mathrm{h}^{-1}$ for 10.000 $\mathrm{mg} \mathrm{chla} \cdot \mathrm{m}^{-3}$ was computed.

Keywords: Light field, culture characterisation, $\mathrm{O}_{2}$ evolution, photosynthesis performance, modelling, Synechocystis 


\subsection{Introduction}

\subsubsection{Light within PBR cultures}

It is a common practice to relate biological experiments with a single number representing irradiance within cell suspensions. In most of the cases, the light source intensity is assumed to be the characteristic value for the culture. Remarkably, this is only a reasonable assumption for biological suspensions at diluted conditions in small vessels, such as cuvettes, where light decay along the optical path-length is minor. Yet, when a PBR is used, this is not necessary the case. Indeed, if the lamp, typically a LED light source with a given arrangement, is not sufficiently wide, the intensity at the corners of the PBR can be significantly lower than at the centre. This complicates the estimation of the real irradiance that cells are exposed to, especially if the homogenisation system is not optimal and hence sub-populations of cells can emerge, whose main characteristics can diverge among each other. Furthermore, once mean irradiance has been properly computed, one can link any physiological property with mean light. However, it can not be discarded that culture responses at same average irradiance but arisen at a different cell density could turn out to account for a slightly different value of that culture characteristic. Nevertheless, it is usual to link PBR properties to mean irradiance in dense cultures Rabe and Benoit (1962) as a simplicity trade-off.

Moreover, cultures in narrow photobioreactors with an appropriate mixing do experience light flashing effect, even under continuous illumination Richmond et al. (2003); Richmond (2008). There are many reports showing that very fast alternations between high light intensities an darkness can improve photosynthesis efficiency dramatically Phillips Jr and Myers (1954); Kok (1953); Nedbal et al. (1996); Terry (1986), since short periods of light do not enhance post-illumination respiratory routes Beardall et al. (1994), photoinhibition Iluz et al. (2012) nor non-photochemical quenching Adams et al. (1999). The main factors affecting photosynthetic performance are the previous cell acclima- 
Chapter 4. Experimental characterisation of Synechocystis sp. PCC 64 6803 cultures productivity up on light conditions

tion to light or dark conditions, the frequency of the light fluctuations and the period of light exposure Grobbelaar et al. (1996). Besides, there are contradictory statements about the effect of extending the dark period with respect to the light phase on the photosynthetic rates Grobbelaar et al. (1996); Kok (1953). Particularly, intermittent illumination in Chlorella vulgaris yields maximal growth rates for switch periods of around $10 \mathrm{~ms}$, whereas $\mathrm{O}_{2}$ evolution was highest at continuous or highfrequency illumination Nedbal et al. (1996).

\subsubsection{Biomass growth rates of Synechocystis cells}

Biomass formation is a critical variable in biotechnological applications, especially for the production of cellular compounds directly linked with cell growth, such as carotenoids or proteins. In this regard, specific growth rates of model organism Synechocystis sp. PCC 6803 have been published in literature, ranging from rather high values (rates equivalent to average doubling times lesser than 5 hours) at very intense dilution rates Touloupakis et al. (2015), intermediate figures of 6-12 hours Lopo et al. (2012); Trautmann et al. (2016) to even longer periods Jiang et al. (2015); Kim et al. (2010), where normally growth velocity is not relevant and the impact of external growing conditions on cell performance is assessed. These essays cover various operational variables, such as light intensity Zavřel et al. (2015); Martínez et al. (2012), carbon species availability Benschop et al. (2003), temperature Červenỳ et al. (2015), pH Touloupakis et al. (2016a), dilution rate Touloupakis et al. (2015), shear stress Sung et al. (2014) or nutrient deficiencies Trautmann et al. (2016); Kim et al. (2015), among others. Apart from differences in the growing conditions, dissimilar illumination set-ups and genetic variability among the strains could explain the differences in growth rates of Synechocystis cultures.

In this research, we have achieved a very high growth of a Synechocystis culture since cells experienced a specific growth rate of $0.15 \mathrm{~h}^{-1}$, corresponding to a doubling time of 4.6 hours (averagely 4.8 hours for all replicates of that series), at the conditions of $0.1 \mathrm{OD}_{720}$ and 220 
$\mu \mathrm{mol}$ photons $\cdot \mathrm{m}^{-2} \cdot \mathrm{s}^{-1}$ of incident continuous red light. As light is a critical variable for a precise photosynthetic yield prediction, we estimated the light profile within the vessel to properly relate all culture properties with average light. In this way, one can work not only with optically thin suspensions, where light can be considered as practically homogeneous, but with dense cultures as the ones utilised in industrial applications.

\section{Chlorophyll a and photosystem ratio of Synechocystis cells}

One of the most utilised techniques to study photosynthetic cultures is the measurement of chlorophyll a as it can provide in a simple manner a general picture of the culture state. However, chlorophyll content depends on many factors, such as the extraction protocol Schagerl and Künzl (2007), the growth medium or the growth phase Knoechel and Quinn (1989) of the cells, but particularly one has the greatest impact: the acclimatisation light intensity. For this reason, plenty of disparate cell chlorophyll estimations can be found, since biological suspensions are exposed to a wide range of light types and intensities in each research.

The chlorophyll content of the here studied cyanobacterium strain is not an exception: the amount of chlorophyll a per Synechocystis sp. PCC 6803 cell described in literature ranges from lower values hardly above $15 \mathrm{fg} \mathrm{chl} \mathrm{a} \cdot$ cell $^{-1}$ Vavilin et al. (2005); Kwon et al. (2013); Ashby and Mullineaux (1999), medium concentrations between 25-60 Tsunoyama et al. (2009), Tsunoyama et al. (2009); Lea-Smith et al. (2014); Kopečná et al. (2012); Hihara et al. (1998); Mohamed et al. (2005) to higher values above those numbers and close to $100 \mathrm{fg} \mathrm{chl} \mathrm{a} \cdot$ cell $^{-1}$ Astier et al. (1984); Moal and Lagoutte (2012); Kirst et al. (2014).

Photosystem I to photosystem II ratio (photosystem (PS) ratio) is another parameter that gives insight into the situation of the photosynthetic machinery, yet its estimation is much more complicated and hence less figures have been reported so far. Such values typically vary between ratios of 4 and 6 Moal and Lagoutte (2012); Shen et al. (1993); 
Chapter 4. Experimental characterisation of Synechocystis sp. PCC 66 6803 cultures productivity up on light conditions

Tian et al. (2011) in Synechocystis strains under low irradiance. Alternatively, ratios lower than two have been also found at very high intensities Murakami and Fujita (1991); Fujita and Murakami (1987). Nevertheless, this proportion is expected to vary up on colour of irradiance Murakami (1997) because cells try to balance energy input arriving the photosystems by modifying the amount of PS. Besides, cyanobacteria reduce their photosystem number under light stress in order to better cope with photodamage Murakami et al. (1997) and in this regard, the principal component down-regulated is photosystem I (PSI) and not photosystem II (PSII) Hihara et al. (1998); Murakami and Fujita (1991) since PSI is more sensitive than PSII to chlorophyll availability $\mathrm{Xu}$ et al. (2004). In fact, strong white light induces a minor up-regulation of PSII and a pronounced one for PSI in Synechocystis sp. PCC 6714 Fujita and Murakami (1987).

\subsubsection{Oxygen related routes of Synechocystis cells}

Similarly situation occurs with reports on $\mathrm{O}_{2}$ evolution. Very different figures have been published for Synechocystis sp. PCC 6803, as it depends on chlorophyll a content, the irradiance that cells are exposed and acclimased to García-Camacho et al. (2012) and the experimental protocol, too. Typical observed values for maximal $\mathrm{O}_{2}$ capacity in Synechocystis are above $250 \mu \mathrm{mol} \mathrm{O} 2 \cdot \mathrm{mg} \mathrm{chl} \mathrm{a}{ }^{-1} \cdot \mathrm{h}^{-1}$ Bernát et al. (2009), some reaching the range of 300-400 units Touloupakis et al. (2015), yet larger rates can also be found Jiang et al. (2015); Shen et al. (1993) following a protocol that uses dichlorobenzoquinone and ferrocyanide Jackson et al. (2014) to investigate PSII real capacity.

The difference between gross and observed (or net) $\mathrm{O}_{2}$ evolution corresponds to the sinks consuming this gas, mainly dark respiration and other routes such generically referred to as photorespiration. The here described photorespiration phenomenon encompasses a series of photosynthesis related mechanisms that take place at higher irradiance values to protect the cell from photodamage by utilising $\mathrm{O}_{2}$ as electron sink. These processes include the phosphoglycolate pathway sustained 
by the RuBP oxygenase function Tolbert (1985), the Mehler reaction driven by the flavodiiron proteins Flv1 and Flv3 Helman et al. (2003), increased respiration under light Falkowski et al. (1985) and other mechanisms involving photodamage Osmond (1994). However, under nonlimiting carbon growth conditions, oxygen reduction in Synechocystis is supposed to be principally mediated by the flavodiiron proteins Allahverdiyeva et al. (2011).

\subsection{Materials and Methods}

\subsubsection{Strain and experimental design}

Synechocystis strain and experimental design, including illumination conditions among others, are identical to the ones used in a previous work Zavřel et al. (2015). The culture parameters were obtained under different incident irradiance values $I_{b, r}$, staying $b$ for the blue intensity and $r$ for the red one in $\mu$ mol photons $\cdot \mathrm{m}^{-2} \cdot \mathrm{s}^{-1}$. In all cases, a small amount of blue radiation was supplied to avoid functional issues possibly arising due to absence of short wavelength light Golden (1995).

\subsubsection{Optical measurements}

In order to have a good estimate on the light intensity emitted by the light source, we measured the radiation at different positions of the reactor glass facing the LED lamp, i.e. real photon flux density reaching the PBR, since there can be a significant spatial heterogeneity of the photon flux emission along the LED lamp de Mooij et al. (2016). Indeed, in our PBR set-up there is a considerable difference among the illumination level at the central and lateral positions: cells located close to the PBR frame, i.e. at any of the four different sides thereof, will experience a total radiation that barely reaches $50 \%$ of the maximal one, which is found in the centre of the reactor (Figure 4.14). Thus, mean light intensity had to be estimated by calculating the irradiance found 
Chapter 4. Experimental characterisation of Synechocystis sp. PCC 68 6803 cultures productivity up on light conditions

at different positions of the reactor glass surface averaged out by the corresponding sub-areas, areas displayed together with their dimensions and surface relative contribution in Figure 4.14.

In this sense we applied the set of intensities used in Zavřel et al. (2015), i.e. $I_{25, r}$ with $r=\{27.5,55,110,220,440,880,1100\}$ for the characterisation of all properties of the cell suspension. However, after a more rigorous calibration procedure, the real mean intensity delivered by the LED lamp turned out to be $74 \%$ of the original value: $I_{19, r}$ and $r=\{20,41,82,163,326,652,815\}$.

To predict the light field within the culture, we utilised a published light model Fuente et al. (2017) that takes into account Inherent Optical Properties (IOPs) Preisendorfer (1961). This mathematical structure was afterwards updated Fuente et al. (2018) by replacing the LambertBeer law with the Mittag-Leffler (ML) equation in order to improve the model accuracy for very low intensities because at light depleted conditions, radiation decay deviates from the pure exponential case. For this reason, the latter model version was the one used to compute any light field property in this contribution and the respective ML tuning parameter is $\alpha=0.995$ as in the original work. To estimate attenuation coefficients Kirk (1984), we measured absorption and scattering spectra of cells grown under all irradiance values. Moreover, contribution of the medium to the light decay, given by its attenuation coefficient $K_{d \_s o l}$ was assumed to be $8.5 \mathrm{~m}^{-1}$ Lea-Smith et al. (2014). For the calibration of emitted light intensity, radiation was measured after the first glass of the reactor, and therefore stays out of the light decay calculation. Yet, the second one contributes to light attenuation, when light intensity is measured on its surface and its equivalent coefficient $K_{d \_ \text {glass }}$ amounts to $5.5 \mathrm{~m}^{-1}$.

To estimate mean irradiance within the PBR, we used that model Fuente et al. (2018) and benchmarked experimental irradiance exiting the PBR culture in the central position with in silico simulations. We compared the estimated attenuation not just at the acclimatisation irradiance $I_{25, r}$, being $r$ the above mentioned intensities but also under just red LED or 
blue LED irradiance. Indeed, all cultures were grown under red light of different intensities supplemented always with the same amount of blue photons. Yet, cells were also momentarily exposed to only red or only blue radiation for this attenuation trial.

Finally, in order to in silico predict light attenuation in very dense cultures beyond the here assessed experimental cell densities, one can assume that the attenuation behaviour lies within the quasi-linear domain Morel and Bricaud (1981a) for the studied cell density range, i.e. situation corresponding to $K_{d}\left(F \cdot c_{s o l}\right)=F \cdot K_{d}\left(c_{\text {sol }}\right)$, being $F$ a scalar factor, and hence we can simply multiply attenuation coefficients by the factor of the chlorophyll density in the suspension. This seems a plausible hypothesis for coefficient magnitudes below $400 \mathrm{~m}^{-1}$, as above this threshold the attenuation tends to approximate a hyperbolic behaviour with respect to cell density Yun and Park (2001). Such limit magnitude is roughly reached for a $\mathrm{OD}_{680}$ density of 5 , which is approximately equivalent to a chlorophyll content of $20 \mathrm{mg} / \mathrm{L}$.

\subsubsection{Model description}

One of the aims of the present contribution is to calculate volumetric $\mathrm{O}_{2}$ generation as a function of the mean irradiance of the culture and the cell density. Knowing such interdependence would allow the elucidation of the maximum productivity of the culture within the PBR and thus predict optimal operational conditions. To this purpose, it was decided to apply a simple but at the same time mechanistic photosynthesis model which could take into account all these features in a straightforward manner, allowing an estimation of $\mathrm{O}_{2}$ evolution under continuous and intermittent light regimes. The model of García-Camacho et al. García-Camacho et al. (2012) covers all these requirements and its equations can be easily implemented. Hence, it was decided to use that mathematical structure as part of the overall simulation procedure. That model copes with the concept of photosynthetic unit (PSU), which is a part of the thylakoid membrane that represents light receptors, essential enzymes and electron carriers required for producing en- 
Chapter 4. Experimental characterisation of Synechocystis sp. PCC

ergetic compounds for the cellular metabolism Mauzerall and Greenbaum (1989). The photosynthesis machinery is assumed to have PSUs that can be found in either resting $x$, activated $x^{*}$ or non-functional $x_{n f}$ state. In this regard, two equations predict the proportion of the latter two PSUs present in the cell, and thus the estimation of the $\mathrm{O}_{2}$ evolution, as a function of the exposure $I$ and the acclimation intensity $I_{0}$, which can be simply equalled to the first one for acclimatised cells (further details can be found in the supplementary material). Nevertheless, a minor change had to be introduced into the model to adapt the formal framework to our strain of study. In our modelling approach, we have assumed that the concept of PSU corresponds to a PSII complex as they are the responsible ones for photosynthetic $\mathrm{O}_{2}$ evolution and in our illumination set-up (orange-red with an additional supply of blue radiation), PSI seems not to be a limiting factor for the electron chain Tyystjärvi et al. (2002). Another difference with regard to the original manuscript is that in this work, the notion of culture-scale has been introduced as we are not considering ideal optically thin suspensions but relatively dense cultures of thousands, whose concentration lies in the range of $\mathrm{mg} \mathrm{chl} \mathrm{a} \cdot \mathrm{m}^{-3}$. In other words, the light profile is not considered as a flat constant intensity but it decays exponentially due to cell absorption, fact that has a strong impact on culture productivity as explained in the results section.

Furthermore, chlorophyll a cellular concentrations and photosystem II are key magnitudes in this modelling approach as they are directly involved in the oxygen evolution. Additionally, they vary because of photo-adaptive processes driven by illumination changes and thus the photosynthesis performance is affected too. To properly model this photoadaptation phenomenon, the proposed modelling framework contains a function correlating irradiance with the photosystem and chlorophyll concentration in the cell through a hyperbolic relationship following the original contribution and are modelled by Equations (4.6) and (4.7). Particularly, at nearly darkness cultivation, photosystem II complexes, and the number of chlorophyll molecules as well, are at highest level, whereas at very high irradiance values, the opposite situation 
rules. Consequently, the maximal and minimal amounts of these variables are required for the in silico procedure. In order to estimate the limits of these parameters, we utilised available information from own experiments and literature data. Taking into consideration all these magnitudes, one can roughly assess the total number of photosystems within a cell. Among the different values for chlorophyll, the maximum amount is relatively easy to gather then very low light conditions, close to the compensation point, are the necessary ones for the cells to possess the maximal concentration of chlorophyll. However, this is not the case for the minimal concentration because at very high intensities, the culture can collapse. For this reason, it was decided to solve the system of Equations (4.8-4.11). Further details are outlined in the supplementary material.

\subsection{Results and discussion}

In this section, the main outcome of the research will be described, which can be divided into three parts: gathered experimental data of main culture characteristics, model parameter estimation and finally the modelling results of $\mathrm{O}_{2}$ productivity.

\subsubsection{Main culture properties}

The main culture features to be assessed are growth, pigment composition and oxygen evolution under different light intensities. Hence, it is first of all necessary to have an appropriate estimation of the mean irradiance the suspension is experiencing during all essays. Following the above described methodology, light decay was modelled by means of IOPs to first reconstruct downward attenuation coefficients $\mathrm{K}_{d}(\lambda)$ (Figure 4.10) that were consequently used to estimate light attenuation and so mean intensity along the optical path-length. In this sense, the computed remaining intensities for the PAR range are in agreement with experimental values (Figure 4.11), which gradually rise from $18 \%$ to $25 \%$, 
Chapter 4. Experimental characterisation of Synechocystis sp. PCC 72 6803 cultures productivity up on light conditions

as at higher growth intensities chlorophyll a cellular content drops considerably due to light stress and so glob/al light absorption is reduced. Only at higher intensities in silico results tend to underestimate attenuation, though the mean relative error for the growth light data (purple light) accounts for just $6 \%$. It is worthwhile noting that such attenuation values correspond to the centre of the reactor surface, so it was assumed that these coefficients rule for the whole PBR surface and deliver mean values ranged between $47 \%$ and $56 \%$ of the incident PAR radiation.

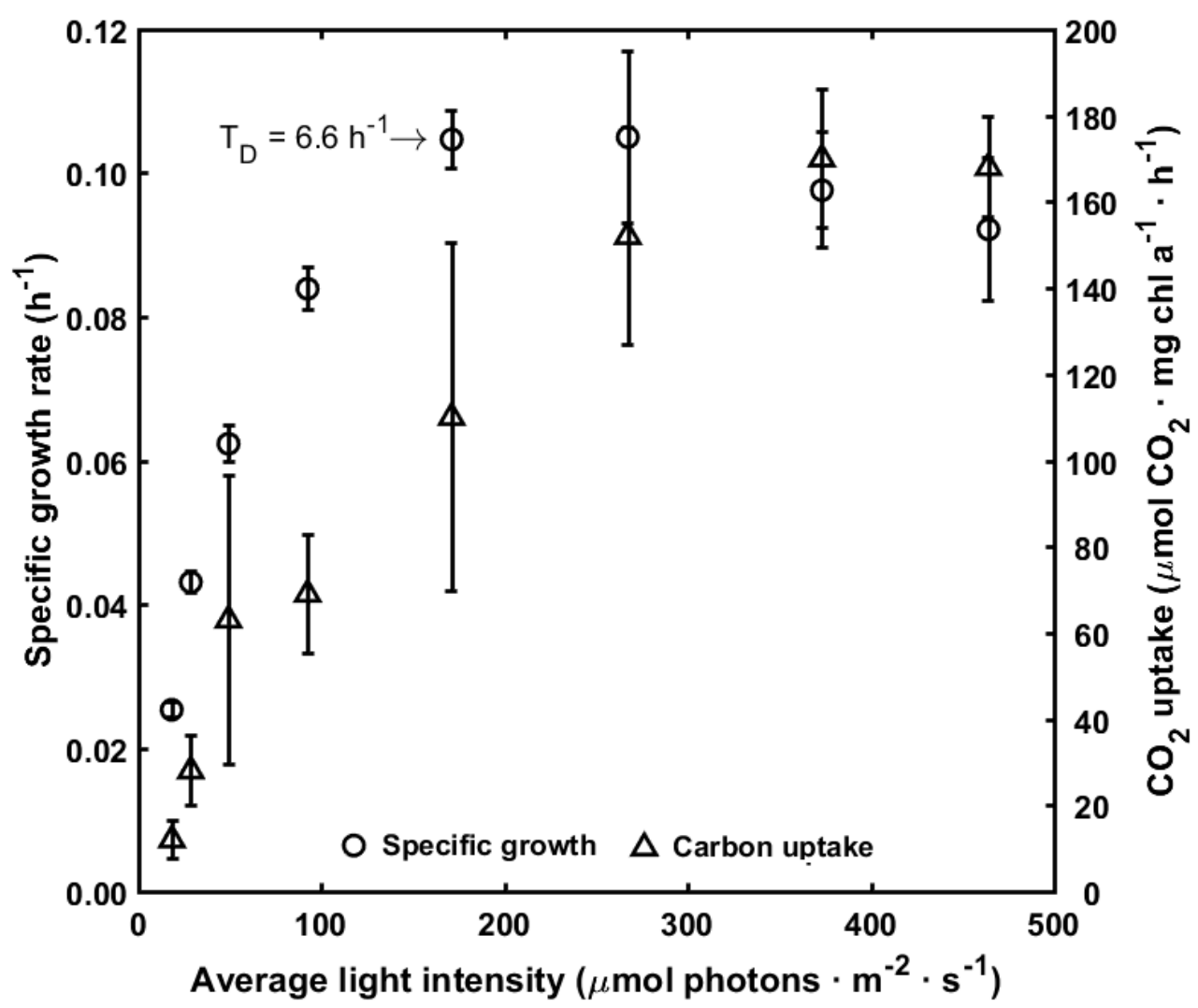

Figure 4.1: Culture specific growth and $\mathrm{CO}_{2}$ uptake under continuous red light. $(\bigcirc)$ markers correspond to the specific growth rate and $(\triangle)$ to carbon uptake. Error bars indicate the standard deviation of the replicates. 
Besides, specific growth measurements under continuous light, growth rates possess a maximum value around $500 \mu \mathrm{mol}$ photons $\cdot \mathrm{m}^{-2} \cdot \mathrm{s}^{-1}$ and then starts to decay due to photoinhibition (Figure 4.1), meanwhile carbon uptake seems to reach its maximal rate at an irradiance which doubles the one for the growth, indicating that part of the carbon fixation goes to other routes, such as carbon accumulation, respiration or photodamage reparation. A minimal doubling time of $6.6 \mathrm{~h}^{-1}$ was reached at irradiance values below $200 \mu \mathrm{mol}$ photons $\cdot \mathrm{m}^{-2} \cdot \mathrm{s}^{-1}$.

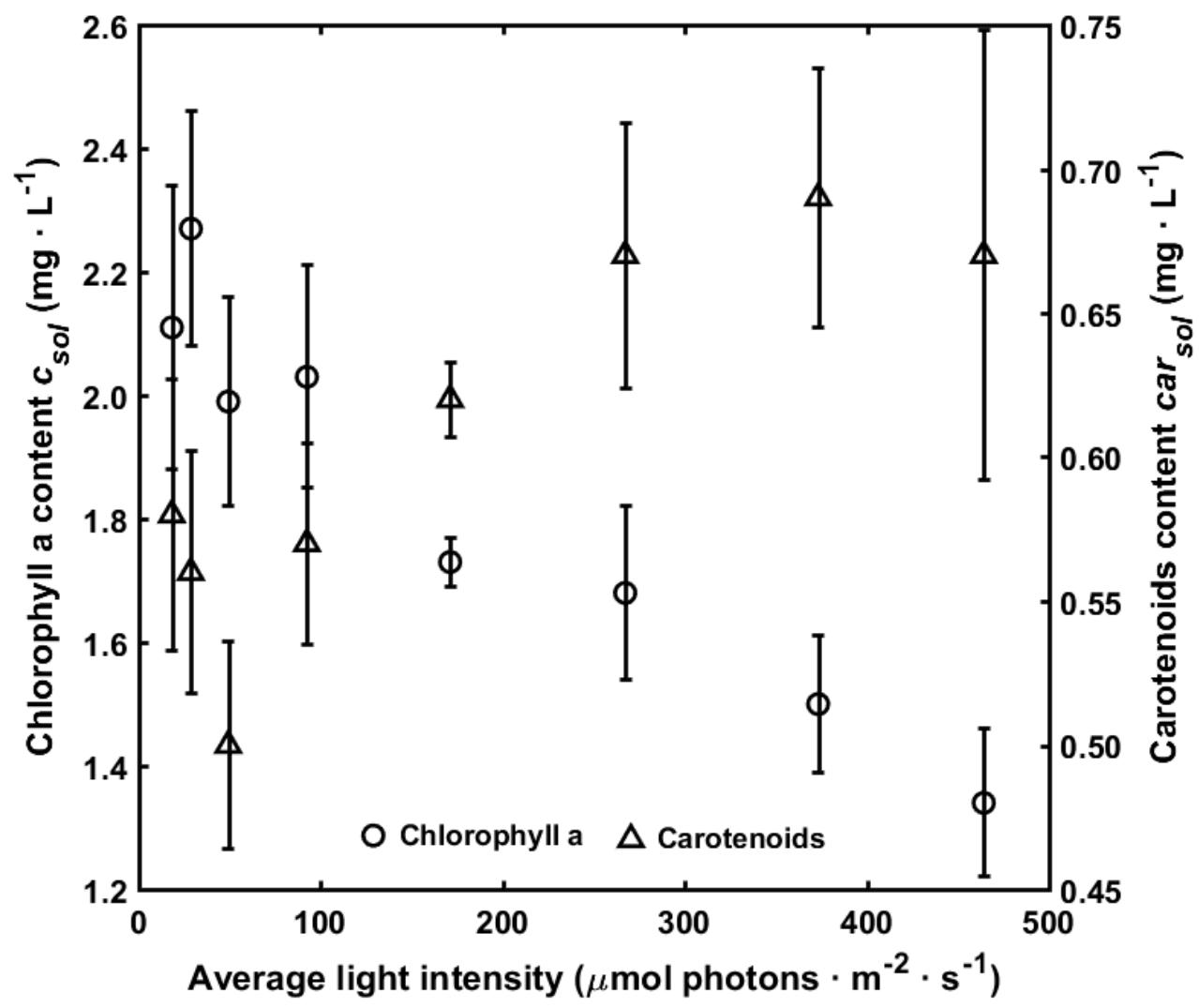

Figure 4.2: Chlorophyll a and carotenoid content under continuous light. $(\bigcirc)$ markers correspond to the the chlorophyll aamount $(\triangle)$ to carotenoidal concentration. Error bars indicate the standard deviation of the replicates.

Pigment composition, chlorophyll content and carotenoids inside the suspension were also measured $\left(\mathrm{mg} \cdot \mathrm{L}^{-1}\right)$ under continuous red light. 
Chapter 4. Experimental characterisation of Synechocystis sp. PCC 6803 cultures productivity up on light conditions

As expected chlorophyll a content follows an inversely proportional relationship to irradiance, whereas carotenoids display a minimal value at low intensities and increase, as light does, to support photoprotection mechanisms. Chlorophyll content drops more than one third along the studied irradiance range and carotenoids sums up to $25 \%$ more concentration under strongest illumination intensity (Figure 4.2).

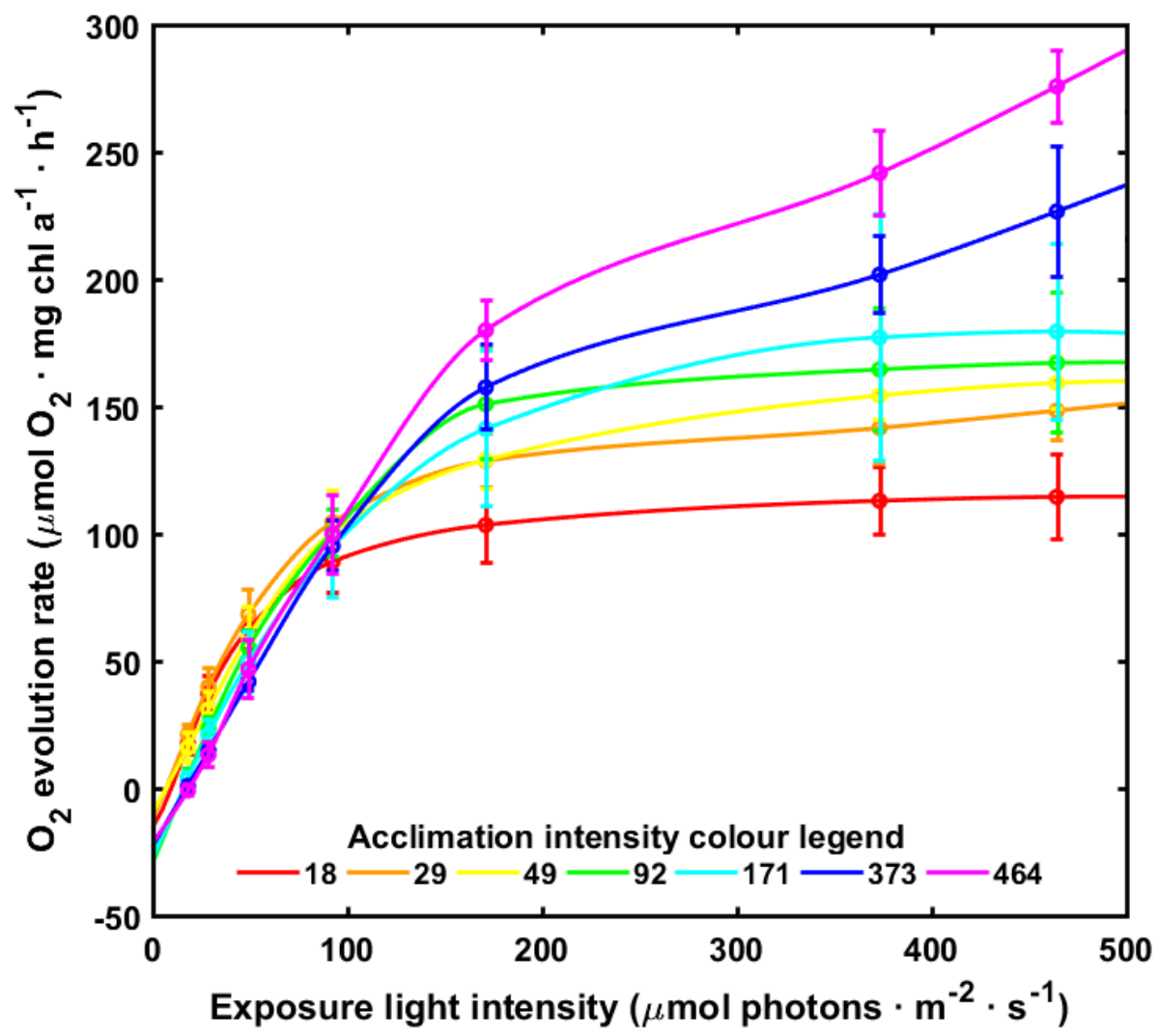

Figure 4.3: Measured $\mathrm{O}_{2}$ evolution at different mean acclimation and exposure light intensities in $\mu$ mol photons $\cdot \mathrm{m}^{-2} \cdot \mathrm{s}^{-1}$ units: $18(-), 29(-), 49(-), 92(-)$, $171(-), 373$ (-) and $464(-)$. Experimental mean values are displayed as dots with error bars, while the corresponding piecewise cubic hermite interpolating polynomial is drawn as a solid curve.

PI curves were measured for obtaining $\mathrm{O}_{2}$ evolution data to benchmark with in silico results and to gain insight into the relationship between photosynthesis and light. $\mathrm{O}_{2}$ generation and dark respiration were as- 
sessed for cells previously adapted to several radiation levels (different colours in Figure 4.3).

In the first case, cells acclimatised to higher light, experience a larger production of the gas, almost tripling the production of the cells adapted to lower intensities, when both cultures are exposed to the highest irradiance in our trials. In the case with the largest generation, net rates reach values close to $280 \mu \mathrm{mol} \mathrm{O}_{2} \cdot \mathrm{mg} \mathrm{chl} \mathrm{a}{ }^{-1} \cdot \mathrm{h}^{-1}$, yet adding the corresponding respiration value, the resulting gross photosynthetic rate sums up to $330 \mu \mathrm{mol} \mathrm{O} 2 \cdot \mathrm{mg} \mathrm{chl} \mathrm{a}{ }^{-1} \cdot \mathrm{h}^{-1}$. Remarkably, the slope of all curves at low exposure intensities is very similar, as light is there a limiting factor. Similarly, respiration is proportional to both, the momentarily exposure intensity and the previous acclimation intensity, as well (Figure 4.4). First, the slope seems to get more negative as the adaptation irradiance does. Moreover, the minimal respiration for each case, i. e. when cells are exposed to nearly dark conditions, is clearly dependent on the acclimation irradiance. Such values are ranged between 8 and $40 \mu \mathrm{mol} \mathrm{O}_{2} \cdot \mathrm{mg} \mathrm{chl} \mathrm{a}^{-1} \cdot \mathrm{h}^{-1}$.

After having analysed the culture under continuous red light, trials with intermittent illumination were carried out to check culture productivity under these conditions. To this purpose, a flashing regime - growth experiment was performed, i. e. periodic cycles of several low light (LL)/high light (HL) red light combinations and at dissimilar flashing frequencies. Blue radiation was supplemented in the same amount as in the case for continuous irradiance.

To allow a proper estimation with respect to continuous illumination experiments, irradiance values for the whole cycle adds up to an average red light intensity of $220 \mu \mathrm{mol}$ photons $\cdot \mathrm{m}^{-2} \cdot \mathrm{s}^{-1}$ for all trials of this series of experiments (Figure 4.6). Continuous light is displayed in this figure as an intermittent period of 0 . The star markers correspond to the continuous illumination set-up of $220 \mu \mathrm{mol}$ photons $\cdot \mathrm{m}^{-2} \cdot \mathrm{s}^{-1}$ of red radiation at $0.1-0.125,0.2-0.225$ and $0.4-0.425 \mathrm{OD}_{720}$ units. In this case, the more diluted the culture, the higher the biomass growth, 
Chapter 4. Experimental characterisation of Synechocystis sp. PCC 6803 cultures productivity up on light conditions

suggesting that for such an intensity cells do not have enough light at denser suspensions.

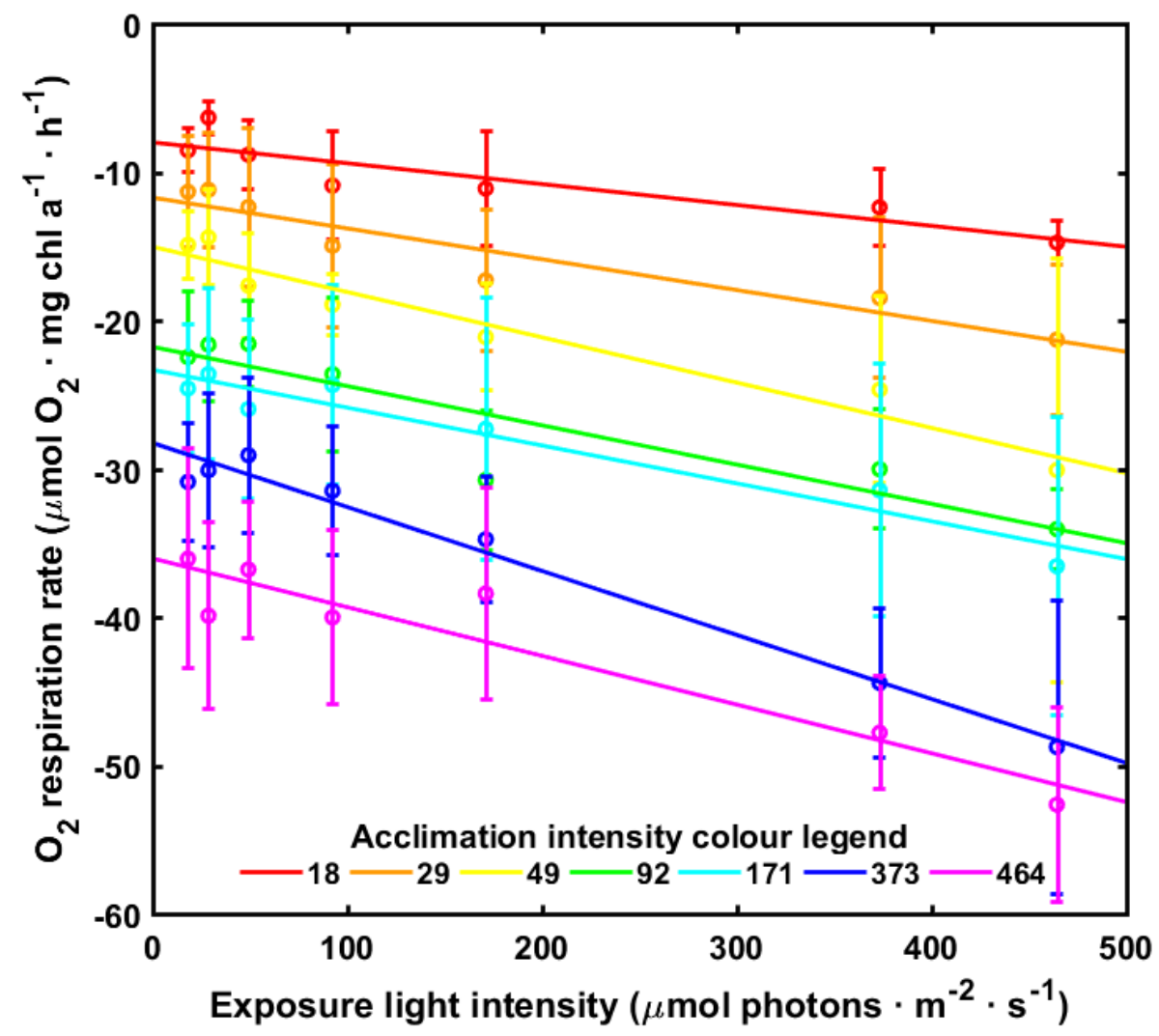

Figure 4.4: Measured dark respiration $\mathrm{O}_{2}$ evolution at different mean acclimation and exposure light intensities in $\mu \mathrm{mol}$ photons $\cdot \mathrm{m}^{-2} \cdot \mathrm{s}^{-1}$ units: $18(-), 29(-)$, $49(-), 92(-), 171(-), 373$ (-) and $464(-)$. Experimental mean values are displayed as dots with error bars, while the corresponding simple linear regression line is drawn as a solid line.

Alternatively, several intermittent illumination trials were performed to cultures whose optical density ranged between 0.1 and $0.11 \mathrm{OD}_{720}$ for all experiments (all markers appearing in Figure 4.6 except starshaped ones). The dashed line with circle-shaped symbols represents the experiment varying the frequency of the light and dark period from $0 \mathrm{~s}$ (no LL phase) to $16 \mathrm{~s}$ for a $40 / 400 \mu \mathrm{mol}$ photons $\cdot \mathrm{m}^{-2} \cdot \mathrm{s}^{-1} \mathrm{LL} / \mathrm{HL}$ mixture. The trend shows an inversely proportional relationship with 
respect to the duration of the switch period, reaching a minimum doubling time of $4.78 \mathrm{~h}^{-1}$ for constant light. Such a high growth rate $\mu$ of $0.15 \mathrm{~h}^{-1}$ is similar to the maximum value reported in literature for any Synechocystis strain, which was achieved at very high dilution rates, close to wash-out conditions Touloupakis et al. (2015). A second row of experiments were carried out at a switch period of $16 \mathrm{~s}$ for several LL/HL combinations: 0/440 -darkness/light conditions- (downwardpointing triangles), 40/400 (circles), 77/363 (squares) and also 110/330 (upward-pointing triangles). The three set-ups with a $16 \mathrm{~s}$ light period are not displayed with an error bar as only a duplicate of each trial is available. In the case of the $16 \mathrm{~s}$ period essays, a higher intensity during the low light period supports a larger cellular growth. Particularly, there is one case, in which the resulting doubling time is closer to the minimal figure reported previously. This could be attributed to the fact that such long exposition periods are far away from operational biochemical processes of photosynthesis and since a lower difference in intensities during the cycle would facilitate cell adaptation to LL/HL alternations.

\subsubsection{Modelled oxygen evolution}

To simulate $\mathrm{O}_{2}$ generation in Synechocystis, model parameters need to be estimated first, either directly gathered via experimental measurements, from previous literature or by fitting in silico results with $\mathrm{O}_{2}$ production data. In this regard, some parameters can be obtained from prior research on this strain, while absorption cross-section and respiratory parameters were measured directly. Particularly, chlorophyll a specific absorption cross-section $\sigma$ follows in our strain a proportional trend with respect to the cellular chlorophyll $\mathrm{c}$ for the studied irradiance range (Figure 4.12), dislike other microorganisms where an inversely proportional behaviour applies Fisher et al. (1989).

To have a direct amount of the chlorophyll present within the cells, one can calculate the concentration per cell as depicted in Figure 4.5. In this case, data show a clear decay with respect to increasing irra- 
Chapter 4. Experimental characterisation of Synechocystis sp. PCC 78 6803 cultures productivity up on light conditions

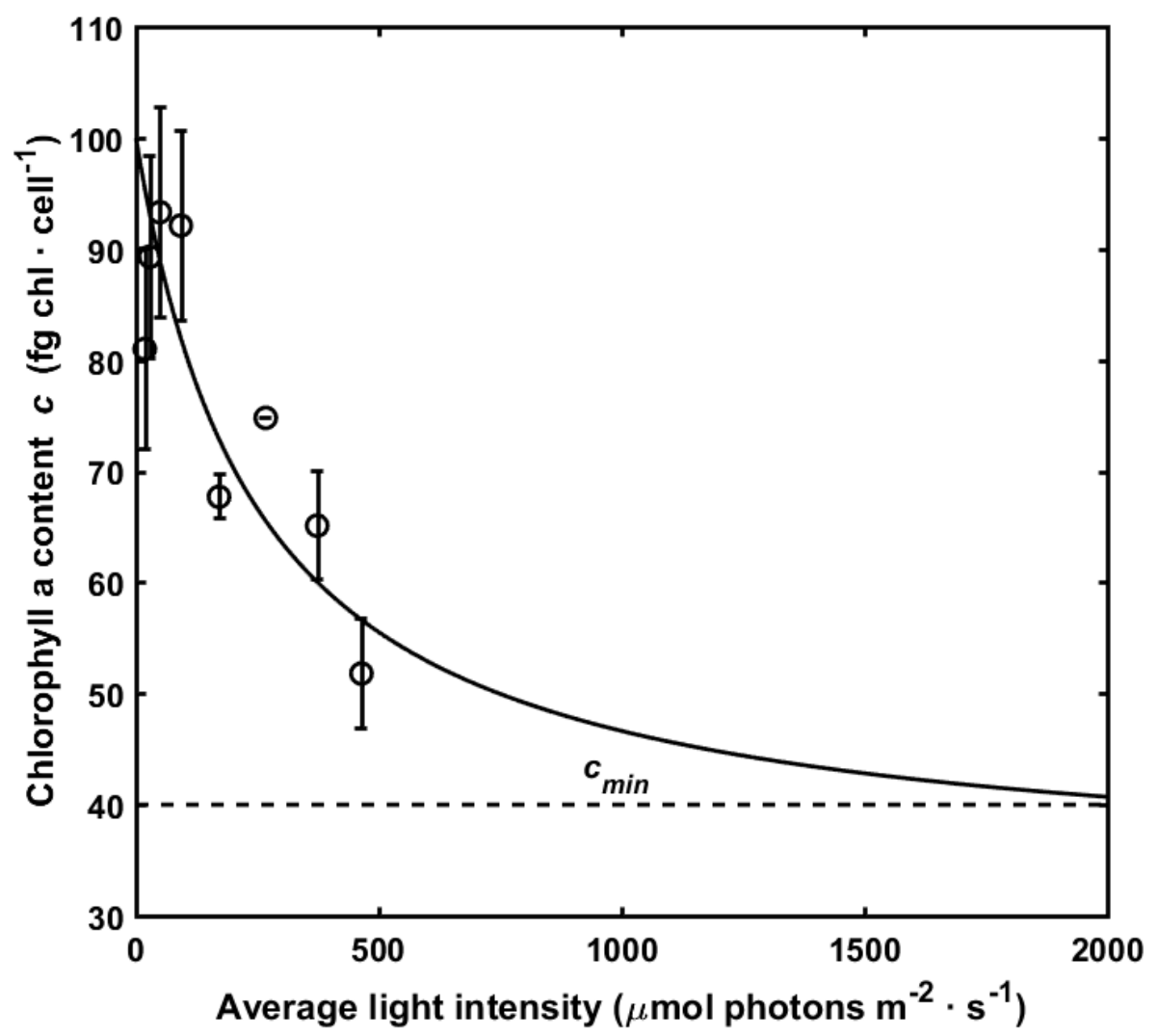

Figure 4.5: Chlorophyll a mass per cell under continuous light. Exponential fit (-) and through a mass balance estimated minimal chlorophyll at saturation intensity (-- -) are also shown.

diance values, starting from values close to $100 \mathrm{fg} \mathrm{chl} \mathrm{a} \cdot \mathrm{cell}^{-1}$ to half of this amount under light saturation conditions. Minimal chlorophyll was estimated from a chlorophyll a-photosystem mass balance system of equations (see supplementary material) to account for around 40 $\mathrm{fg}$, number that seems to be a good guess according to the exponential fit of the experimental measurements. It is noteworthy to remark that this minimal concentration is a theoretical threshold for cells under very high light intensities, yet cultures grown under extreme radiation would probably collapse. 


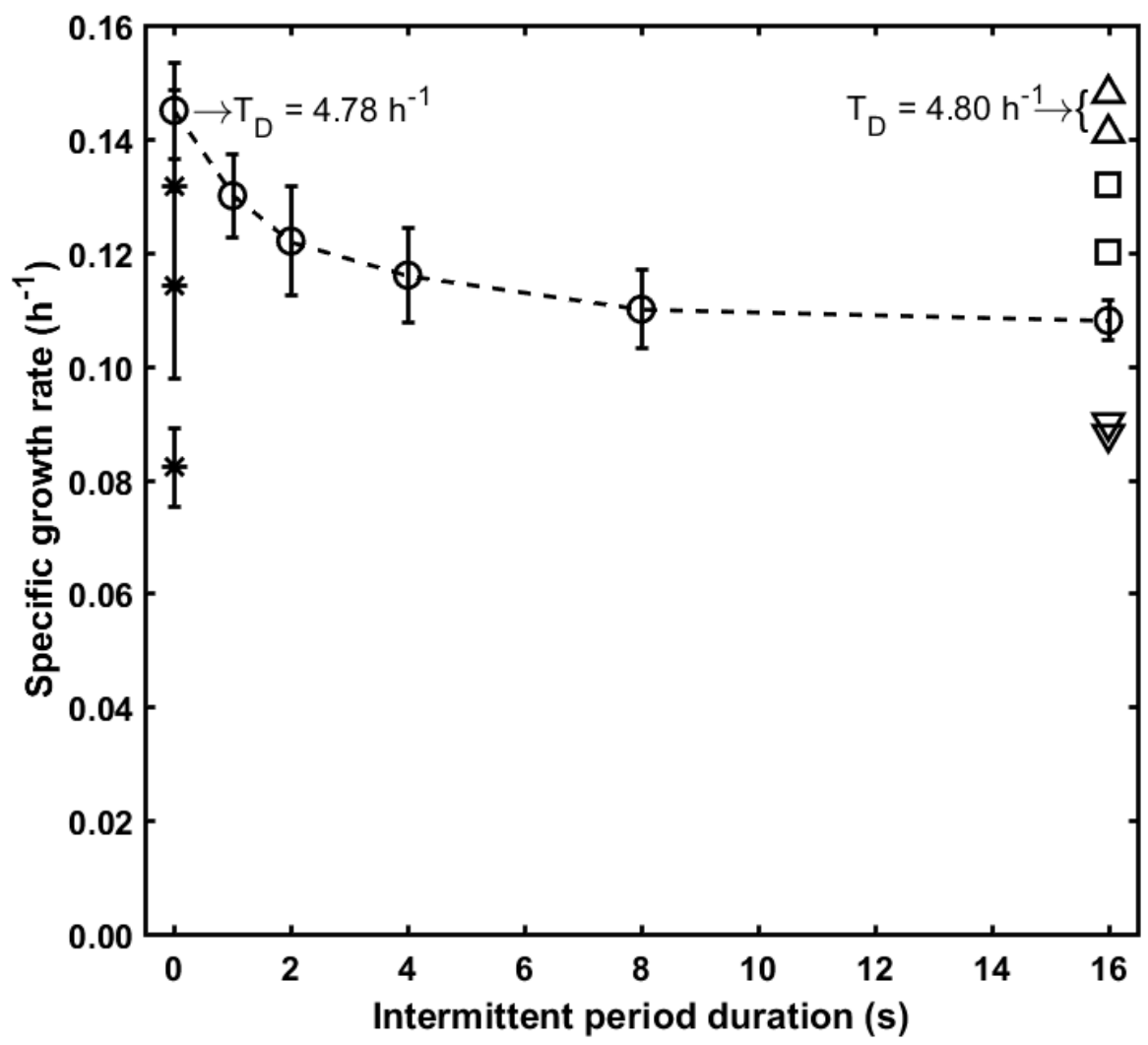

Figure 4.6: Specific growth under different optical density values, light intensity combinations and frequency values. Star markers (*) represent cultures under 220 $\mu$ mol photons $\cdot m^{-2} \cdot s^{-1}$ continuous red light at 0.1, 0.2 and $0.4 \mathrm{OD}_{720}$ (the lower density, the higher the growth) and for the sake of convenience, they are plotted under the zero duration period. Circles display $(\bigcirc)$ cultures at $0.1 \mathrm{OD}_{720}$ with dissimilar switch frequencies with a red light combination of LL/HL 40/400 $\mu$ mol photons $\cdot m^{-2} \cdot s^{-1}$. The other symbols represent duplicated cultures of 0.1 $\mathrm{OD}_{720}$ with an alternation frequency of $16 \mathrm{~s}$, whereas upward-pointing triangles $(\triangle)$, squares $(\square)$ and downward-pointing triangles $(\nabla)$ depict the following combination of LL/HL intensities: $110 / 330,77 / 363,0 / 440 \mu \mathrm{mol}$ photons $\cdot \mathrm{m}^{-2} \cdot \mathrm{s}^{-1}$. Lowest doubling times indicated.

Regarding the relation between the dark respiration and the observed oxygen evolution, as expected, it also follows a linear behaviour. Dark respiration accounts for roughly $13 \%$ of the oxygen generation at a 
given acclimation irradiance (Figure 4.13). Noticeably, dark respiration in cells that have been acclimatised to nearly darkness conditions is almost zero.

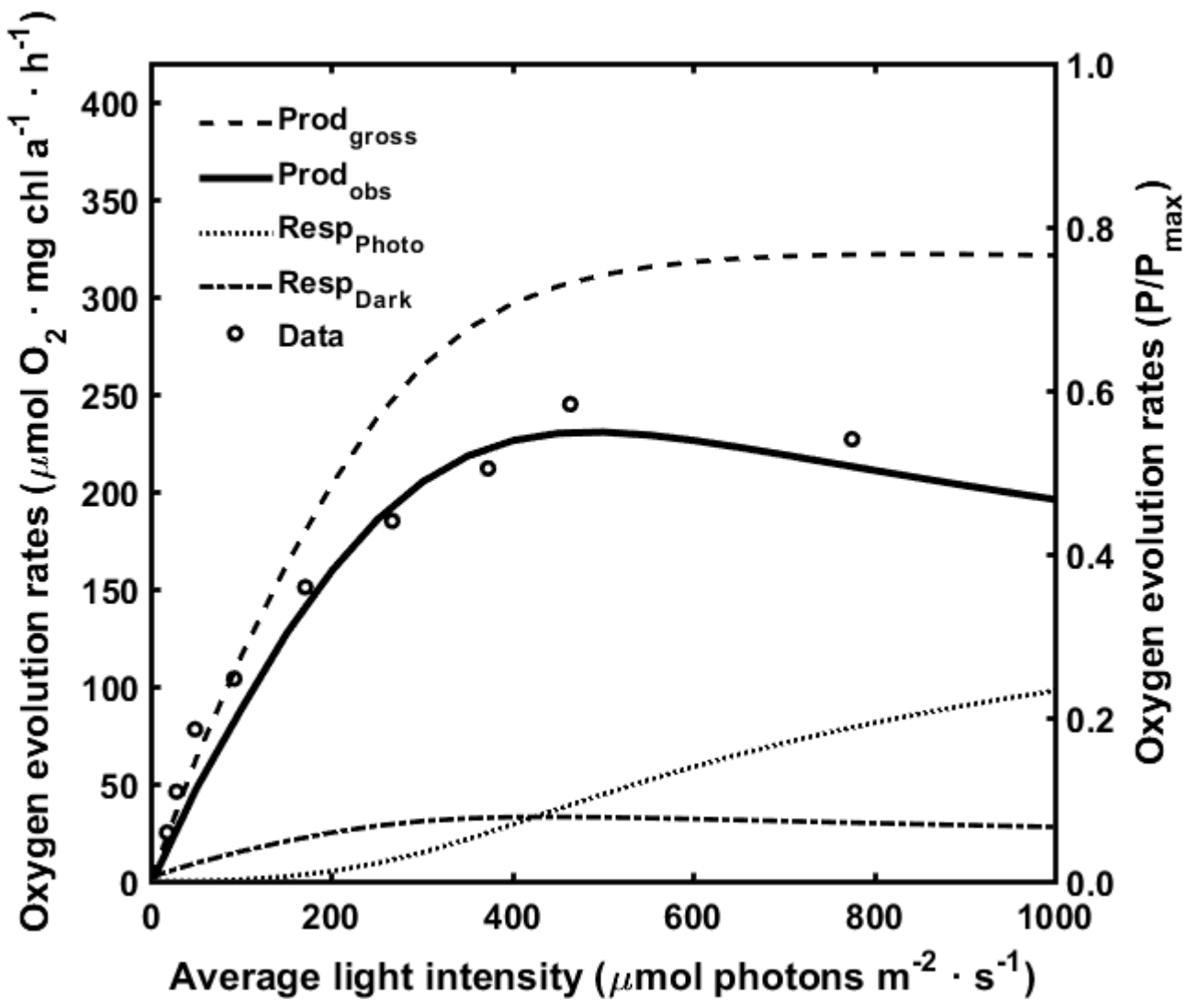

Figure 4.7: Steady state oxygen evolution $\left(\mu \mathrm{mol} \mathrm{O}_{2} \cdot \mathrm{mg} \mathrm{chl} \mathrm{a}^{-1} \cdot \mathrm{h}^{-1}\right)$ with respect to average irradiance. Dots correspond to experimental value, while lines depict the modelled outcome: gross photosynthetic $\mathrm{O}_{2}$ rates $P_{\text {gross }}(-)$, related net photosynthesis performance $P_{n e t}(---)$, dark respiration $R_{\text {dark }}(-\cdot-)$ and photo-respiration $R_{\text {photo }}(\cdots \cdots)$.

The next step for estimating overall culture capacity in terms of $\mathrm{O}_{2}$ evolution was the selection of the appropriate parameters' magnitude to match observed photosynthetic $\mathrm{O}_{2}$ rates. The corresponding result (continuous line in Figure 4.7) shows some discrepancy regarding the shape of the modelled curve and the data, yet the general fit is reasonable. 


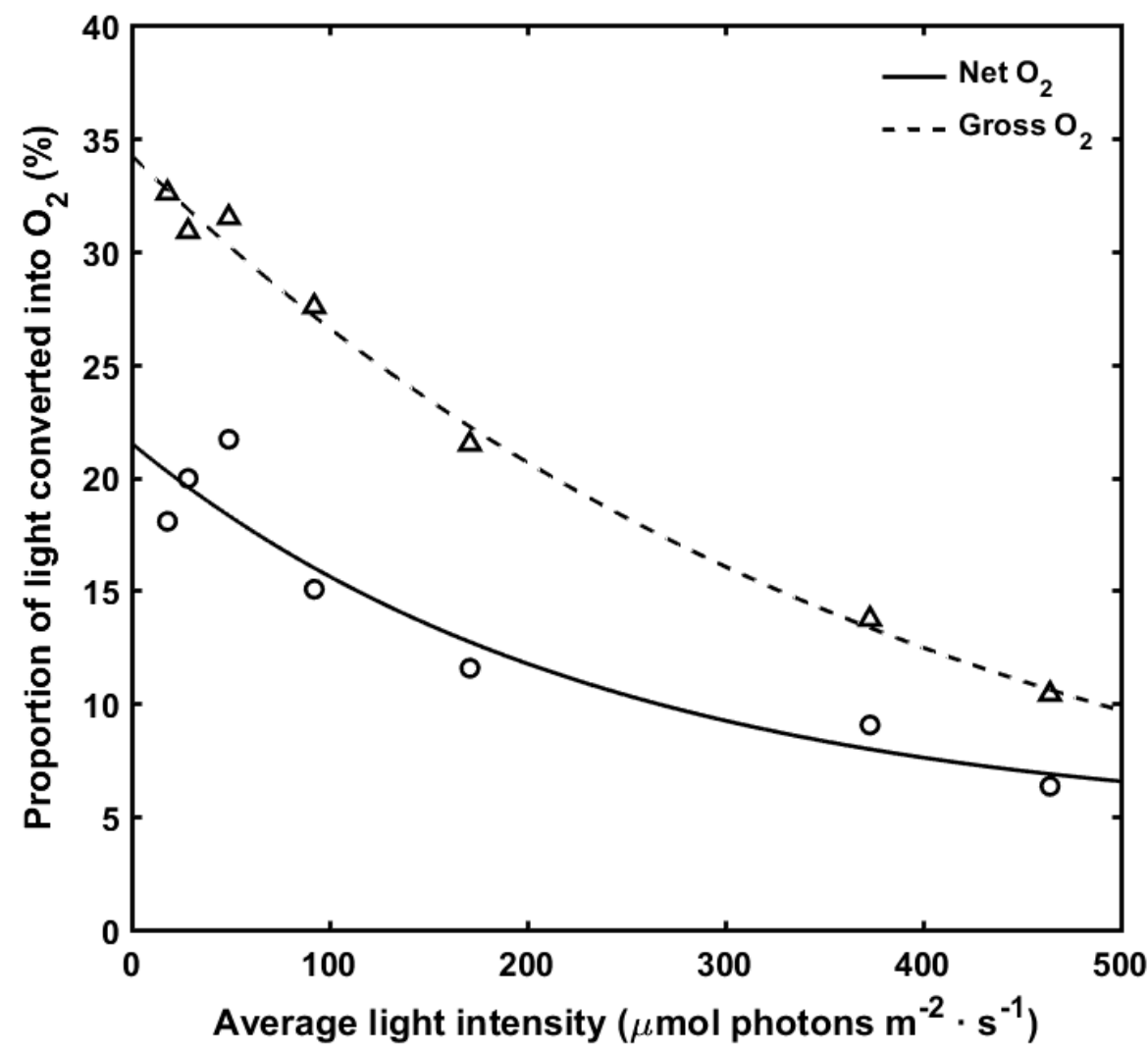

Figure 4.8: Proportion of absorbed light transformed into $\mathrm{O}_{2}$ molecules, net (-) and gross (- -) productivity. 
Chapter 4. Experimental characterisation of Synechocystis sp. PCC

This suggests that the proposed model is not able to perfectly describe our experimental measurements, though the degrees of freedom, since many parameters were obtained by fitting the data. Remarkably, the other processes seem to be realistically approached as they are comparable to other experimental results. Particularly, Synechocystis can yield $\mathrm{O}_{2}$ rates as high as our estimated gross productivity (dashed line), which is close to $350 \mu \mathrm{mol} \mathrm{O} \cdot \mathrm{mg} \mathrm{chl} \mathrm{a} \mathrm{a}^{-1} \cdot \mathrm{h}^{-1}$ under strong illumination Shen et al. (1993); Wang et al. (2010). Besides, dark respiration to net $\mathrm{O}_{2}$ proportions averagely accounts for $13 \%$ (Figure 4.13), value in complete agreement in this strain Touloupakis et al. (2015). Moreover, the photorespiratory procedure, which is assumed to be mostly articulated by the flavodiiron proteins Flv1/3, can sum thrice the respiration in darkness Ermakova et al. (2016) hand this is our case for intermediate to intense radiation levels (dotted and dash-dotted curves, respectively).

Cell productivity can also be calculated as a function of the total photon flux captured that has been transformed into $\mathrm{O}_{2}$ as an estimation of the electron chain flow and hence of photosynthesis performance. In this regard, assuming a quantum yield of $0.125 \mathrm{O}_{2}$ per photon, a relative photosynthesis output can be computed (Figure 4.8). In this sense, even at low intensities, performance is below $35 \%$ and $20 \%$ for the gross and net productivity, respectively. As irradiance increases, photosynthesis gets saturated and therefore efficiency diminished till values ranged between $7 \%$ and $10 \%$.

Further, one can calculate the capacity of not only a single cell but of the whole culture. In this case, more aspects need to be taken into consideration as cellular concentration has to be integrated into the calculation. We computed the volumetric yield of the suspension given in the units of $\mu \mathrm{mol} \mathrm{O}{ }_{2} \cdot \mathrm{L}^{-1} \cdot \mathrm{h}^{-1}$ for a wide range of cell densities $\left(\mathrm{mg} \mathrm{chl} \mathrm{a} \cdot \mathrm{m}^{-3}\right)$ acclimatised at different incident growth intensities as displayed in Figure 4.9. As stated, light attenuation in dense suspensions is rather step, so in order to obtain a more precise estimation of the $\mathrm{O}_{2}$ production and assuming that cells are located randomly along the optical path-length, cell location was discretised into in 11 equidistant positions along the 
optical path-length and thus exposed to 11 irradiance levels, i.e. some cells close to the light source will experience very strong intensity, others very little, when situated far from the LED lamp.

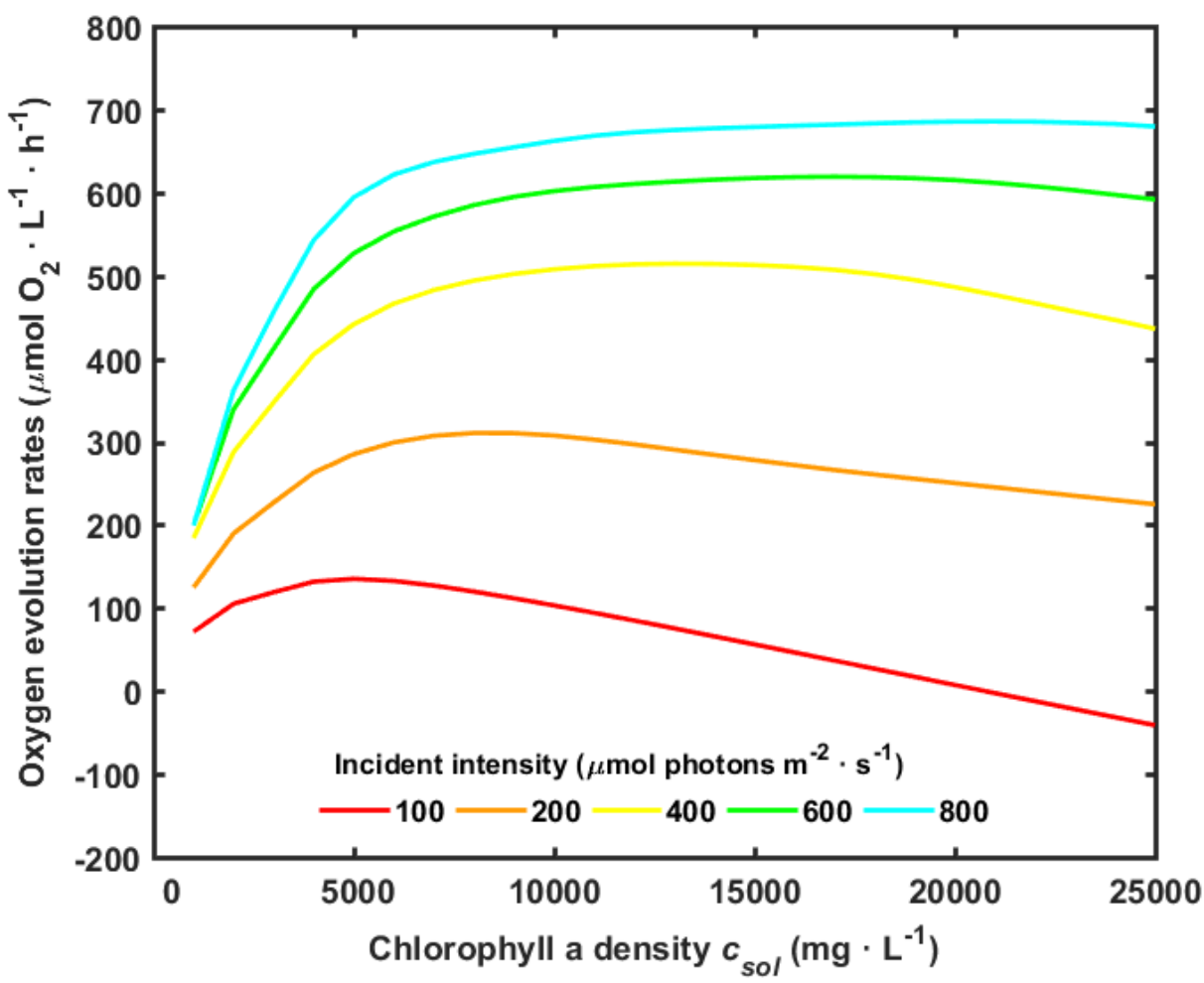

Figure 4.9: Modelled $\mathrm{O}_{2}$ generation $\left(\mu \mathrm{mol} \mathrm{O} \mathrm{O}_{2} \cdot \mathrm{L}^{-1} \cdot \mathrm{h}^{-1}\right.$ ) with respect to the culture chlorophyll a concentration $\left(\mathrm{mg} \cdot \mathrm{m}^{-3}\right)$ at different growth light intensities. Simulations of cultures acclimatised to the following incident intensities in $\mu$ mol photons $\cdot m^{-2} \cdot s^{-1}$ are plotted: light intensities in $\mu$ mol photons $\cdot m^{-2} \cdot s^{-1}$ units: $100(-), 200(-), 400(-), 600(-), 800(-) . \mathrm{O}_{2}$ volumetric production results from a single average acclimation intensity, but cells situated at then different equidistant positions along the optical path.

In other words, this approach considers that cells are continuously moving and are exposed to very high intensities when the movement of the PBR fluid brings them closer to the lamp and smaller ones when cells face the opposite situation. Regarding the light intensities studied, the lowest growth irradiance corresponds to $100 \mu \mathrm{mol}$ photons $\cdot \mathrm{m}^{-2} \cdot \mathrm{s}^{-1}$ 
Chapter 4. Experimental characterisation of Synechocystis sp. PCC

of incident radiation, while the others are stepwise increments up to $800 \mu \mathrm{mol}$ photons $\cdot \mathrm{m}^{-2} \cdot \mathrm{s}^{-1}$. The curves show a maximum productivity around $4.000 \mathrm{mg} \mathrm{chl} \mathrm{a} \cdot \mathrm{m}^{-3}$ at the lowest intensity that progressively rises up to values of chlorophyll a close to 20.000 units. This is due to the fact that in cultures with high cell densities, light decay is rather pronounced, so intense irradiance is required for larger productivities. A remarkable feature of the model is that the slopes for very dense suspensions are proportional to the respiratory rates, mainly dark respiration. Moreover, such slopes are very small as the model supposes low respiration for dense cultures due to non-availability of light. Nevertheless, experimental data present stronger productivity drops at ultradense cultures, especially under strong illumination Kwon et al. (2013), suggesting that respiratory process are much enhanced when cells are exposed to large irradiance values even if the mean radiation is very low as for the case of concentrated suspensions.

\subsection{Conclusions}

In this contribution, we have carried out a characterisation of Synechocystis sp. PCC 6803 GT strain regarding main cultures properties as a function of the real average radiation cells are experiencing inside the PBR. Other main parameters of the cell such as photosystem ratio, chlorophyll aper cell limits and optical properties were computed too. Besides, $\mathrm{O}_{2}$ as an indicator of photosynthesis performance was assessed: optimal irradiance values for $\mathrm{O}_{2}$ productivity were obtained at cellular scale and for different chlorophyll aconcentrations at culture scale, as well. Future research will deal with more detailed electron chain processes modelling applied to optical antenna mutants that are able to optimise light fields at culture scale within high cell-density suspensions. 


\section{Supplementary material}

\section{Model description}

The original model García-Camacho et al. (2012) comprises two equations that predict the steady-state proportion of the activated $x^{*}$ and non-functional $x_{n f}$ PSUs present in the cell, given by Equations (4.1) and (4.2) respectively. These variable are a function of the momentarily exposure intensity $I$, whereas the rest of parameters of the system depend on the number of PSUs $a$ and the chlorophyll a content $c$, which are supposed to vary in a long-term range and thus depend not on $I$ but on the previous acclimation radiation $I_{0}$. For photoacclimated cells, both light intensities are the same. The equations are the following:

$$
\left[\left(1-x^{*}(I)-x_{n f}(I)\right]-\frac{\left(\alpha\left(I_{0}\right) / I\right) \cdot x^{*}(I)}{\kappa\left(I_{0}\right)+x^{*}(I)}-\frac{\kappa_{i}\left(I, I_{0}\right) \cdot a\left(I_{0}\right) \cdot \eta\left(I_{0}\right)}{\xi\left(I_{0}\right) \cdot I} x^{*}(I)=0\right.
$$

$\kappa_{i}\left(I, I_{0}\right) \cdot x^{*}(I)-\frac{\kappa_{r}\left(I_{0}\right) \cdot x_{n f}(I)}{\kappa_{n f}\left(I_{0}\right)+x_{n f}(I)}=0$

In our work, we have considered $a$ as the total number of PSII units, excluding PSI complexes, as the former are the responsible for the $\mathrm{O}_{2}$ evolution by means of the water photolysis and are supposed to be the photosystem type limiting the whole process under white light. The pseudo-constants are in turn defined by more fundamental parameters:

$$
\begin{gathered}
\alpha\left(I_{0}\right)=\frac{\eta \cdot r_{m}^{*}}{\xi\left(I_{0}\right)}, \quad \kappa\left(I_{0}\right)=\frac{K_{S}^{*}}{a\left(I_{0}\right)}, \quad \kappa_{r}\left(I_{0}\right)=\frac{k_{r}}{a\left(I_{0}\right)}, \\
\kappa_{n f}\left(I_{0}\right)=\frac{k_{n f}}{a\left(I_{0}\right)}, \quad \kappa_{i}\left(I, I_{0}\right)=k_{i} \xi\left(I_{0}\right) I^{n}
\end{gathered}
$$


Chapter 4. Experimental characterisation of Synechocystis sp. PCC 86 6803 cultures productivity up on light conditions

Additionally, $\xi\left(I_{0}\right)$ is another important parameter, the cellular absorption cross-section, and relies on the cellular chlorophyll content per cell $c$. In this work and following the linear trend displayed by the experimental data (Figure 4.12), this relationship has been particularly modelled in this way:

$$
\xi\left(I_{0}\right)=\xi_{\min }+m_{\xi} \cdot c\left(I_{0}\right)
$$

Finally, the relationship of the cellular chlorophyll $c$ and total PSU amount $a$ are described as follows:

$$
\begin{aligned}
& c\left(I_{0}\right)=c_{\text {max }}-\left(c_{\text {max }}-c_{\text {min }}\right) \cdot \frac{I_{0}}{I_{c}+I_{0}} \\
& a\left(I_{0}\right)=a_{\text {max }}+\left(a_{\text {max }}-a_{\text {min }}\right) \cdot \frac{I_{0}}{I_{a}+I_{0}}
\end{aligned}
$$

Being $c_{\max }, a_{\max }$ known figures at light intensities close to zero and $c_{\text {min }}, a_{\text {min }}$ uncertain values under very high radiation. In order to estimate $c_{\text {min }}$ and $a_{m i n}$, one has to solve the following chlorophyll mass balance equations, assessing chlorophyll a presence in both type of photosystems:

$$
\begin{aligned}
P S_{\text {ratio }} & =P S I(\max ) / P S I I(\min ) \\
Q_{X} & =X(\max ) / X(\min ), \quad X=P S I, P S I I \\
c_{\text {min }} & =P S I I(\min ) \cdot c_{P S I I}+P S I(\min ) \cdot c_{P S I} \\
& =P S I I(\max ) / Q_{P S I I} \cdot c_{P S I I}+P S_{\text {ratio }} \cdot P S I I(\max ) / Q_{P S I} \cdot c_{P S I}
\end{aligned}
$$

$$
\begin{aligned}
c_{\max } & =P S I I(\max ) \cdot c_{P S I I}+P S I(\max ) \cdot c_{P S I} \\
& =P S I I(\max ) \cdot c_{P S I I}+P S_{\text {ratio }} \cdot P S I I(\min ) \cdot c_{P S I}
\end{aligned}
$$


$P S_{\text {ratio }}$ represents the photosystem ratio at low light conditions, while $Q_{P S I}$ and $Q_{P S I I}$ are the PSI and PSII proportions between their maximum and minimum values along the whole irradiance range. The chlorophyll a molecules associated with each photosystem correspond to $c_{P S I}$ and $c_{P S I I}$. Most of the chlorophyll in Synechocystis is associated with PSI as each monomer contains 96 molecules of chlorophyll, while a PSII structure only possesses 35 Jordan et al. (2001). Moreover, assuming that $c_{\max }=100 \mathrm{fg} \mathrm{chl} \mathrm{a} \cdot \mathrm{cell}^{-1}$, as experimentally measured, and PSII $(\max )=1.2 \cdot 10^{5}$ PSII per cell (adapted from Moal and Lagoutte (2012) to our $c$ ), one can solve Equation (4.11) and obtain a photosystem ratio of 5.5, which is in alignment with previous reports in this strain Moal and Lagoutte (2012); Shen et al. (1993); Tian et al. (2011). Additionally, if one hypothesises that $Q_{P S I}=2.7$ Fujita and Murakami (1987), Equation (4.10) does only depend on the unknown parameters $c_{\text {min }}$ and $Q_{P S I I}$. Hence, the system of equations is underdetermined and it is necessary to give values to one of these parameters to obtain a solution. Finally, supposing $Q_{P S I}$ to be 1.2 Fujita and Murakami (1987), $c_{\text {min }}$ results in $40 \mathrm{fg}$ chl a $\cdot$ cell $^{-1}$. For a wide range of $Q_{P S I I}$ values (0.5-1.5), the minimal chlorophyll solution is in any case located between 38 and $44 \mathrm{fg} \mathrm{chl} \mathrm{a} \cdot$ cell $^{-1}$. Such numbers are just slightly lower than our experimental values (Figure 4.5). In summary, for a $20 \%$ decrease of PSII under strong illumination, a minimal chlorophyll a content of $40 \mathrm{fg} \mathrm{chl} \mathrm{a \cdot cell}{ }^{-1}$ and $1.0 \cdot 10^{5}$ PSII complexes will be assumed for the modelling framework. Besides, the proposed $Q_{P S I I}$ and $Q_{P S I}$ lead to a PS ratio at very high light conditions of 2.4, estimation which concurs well with prior works Murakami and Fujita (1991); Fujita and Murakami (1987).

The activated and non-functional PSU determine the state of the cell and the different oxygen related processes, net and gross photosynthetic $\mathrm{O}_{2}$ evolution, dark- and photo-respiratory mechanisms: 
Chapter 4. Experimental characterisation of Synechocystis sp. PCC 88 6803 cultures productivity up on light conditions

$$
\begin{aligned}
P_{\text {net }} & =\frac{P_{m} \cdot x^{*}}{\kappa+x^{*}} \\
P_{\text {gross }} & =\frac{1}{1+\gamma}\left(\frac{P_{m} \cdot x^{*}}{\kappa+x^{*}}-m-R_{\text {photo }}\right) \\
R_{\text {dark }} & =m+\gamma \cdot P_{o b s} \\
R_{\text {photo }} & =\frac{R_{m} \cdot\left(x^{*}+x_{n f}\right)}{k_{p h}+\left(x^{*}+x_{n f}\right)}
\end{aligned}
$$




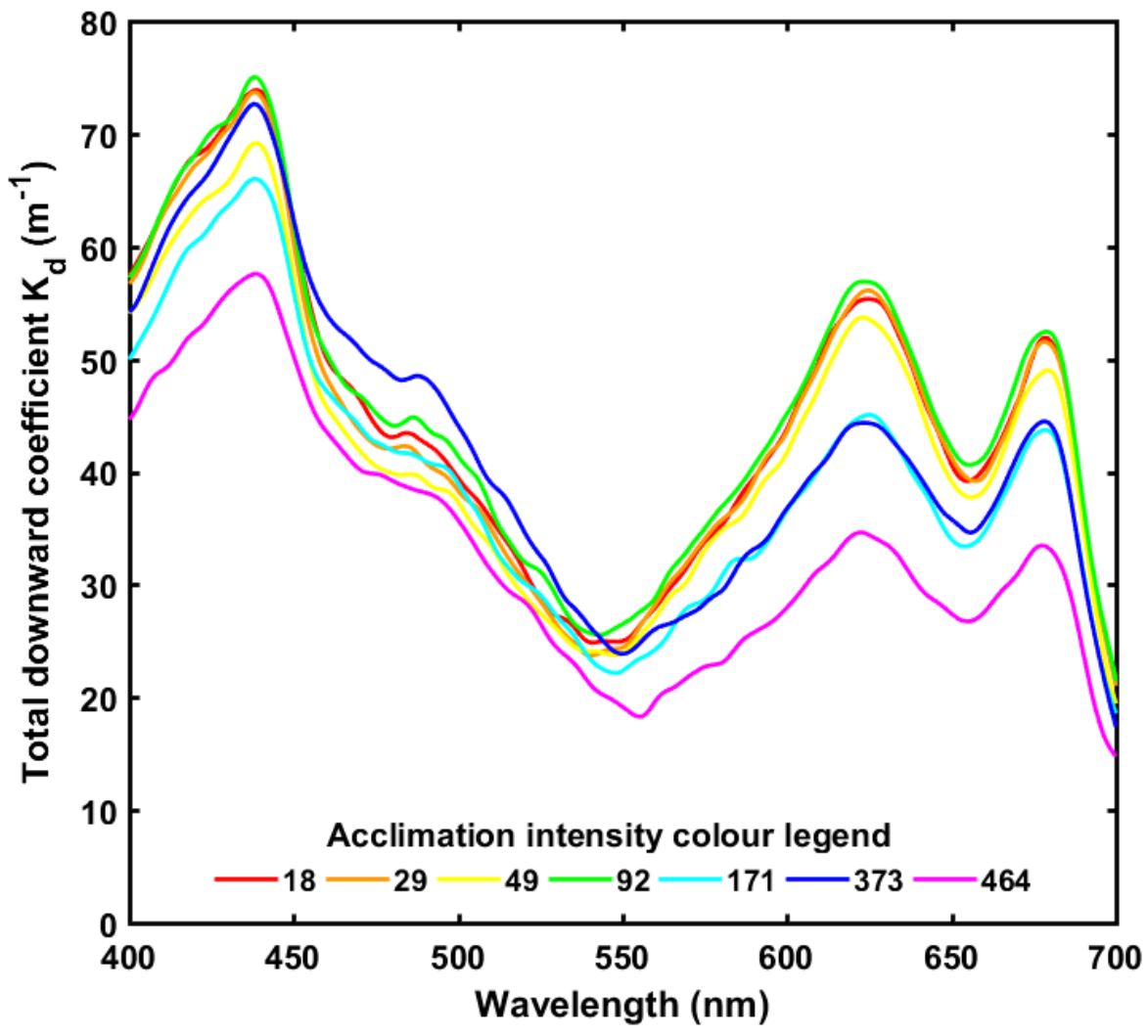

Figure 4.10: Downward coefficients $K_{d}$ in $m^{-1}$ at different average growth intensities gathered via IOPs calculation from experimental absorption and scattering spectra at the given intensities: light intensities in $\mu$ mol photons $\cdot m^{-2} \cdot s^{-1}$ units: 18 (一), $29(-), 49(-), 92(-), 171(-), 373(-)$ and $464(-)$. 


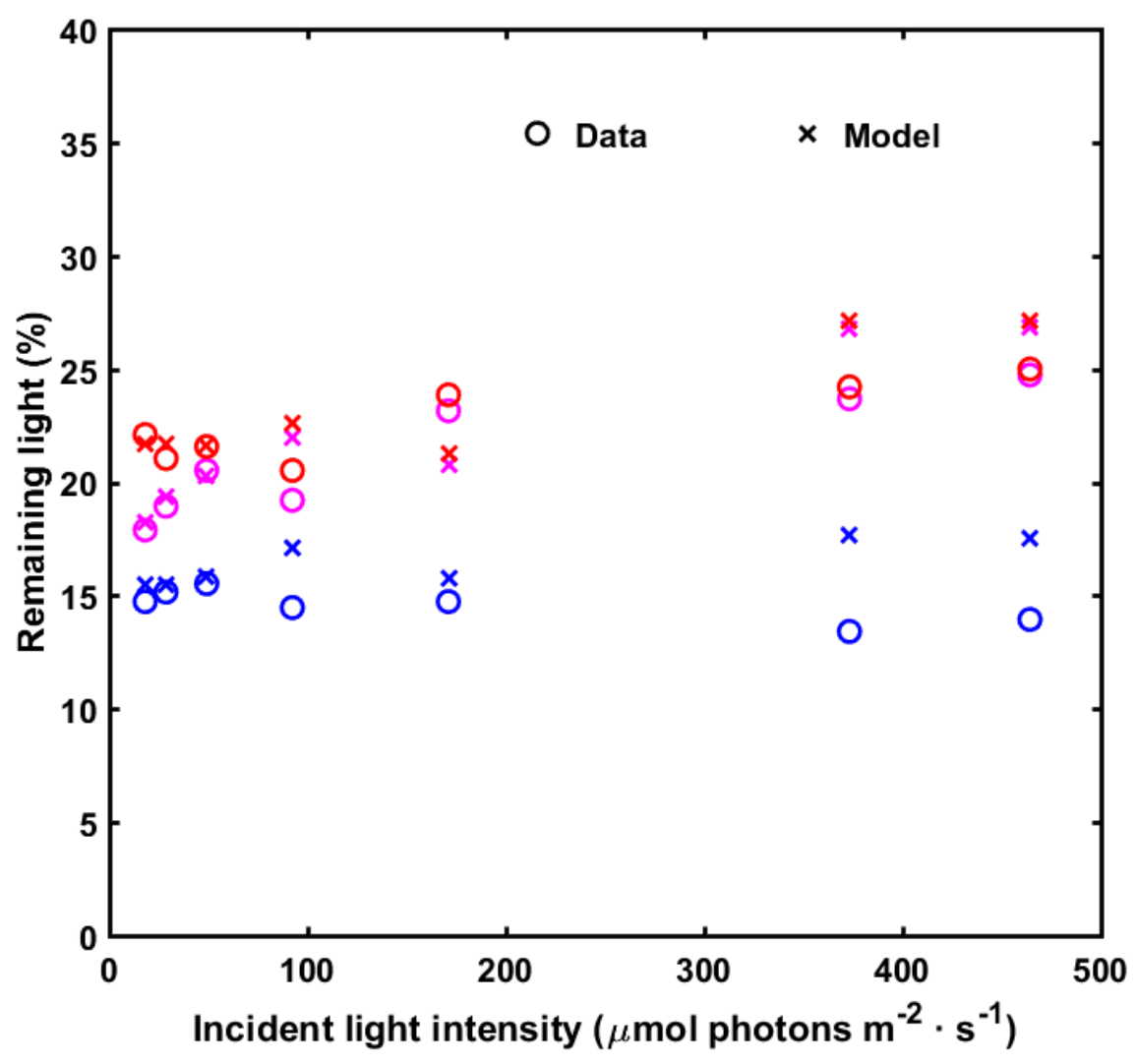

Figure 4.11: Remaining light intensity leaving the PBR at the centre of the reactor surface $(z=2.4 \mathrm{~cm})$ with respect to the incident one for cultures grown at different red light intensities supplemented with $25 \mu \mathrm{mol}$ photons $\cdot \mathrm{m}^{-2} \cdot \mathrm{s}^{-1}$ of blue radiation. The colour represents the momentarily exposure intensity: blue one indicates momentary pure blue exposure $\left(25 \mu \mathrm{mol}\right.$ photons $\left.\cdot \mathrm{m}^{-2} \cdot \mathrm{s}^{-1}\right)$, red one implies only red exposure without addition of blue photons and purple colour corresponds to the original bluered growth light. $(\bigcirc)$ markers represent measured data and $(x)$ ones correspond to modelled values. 


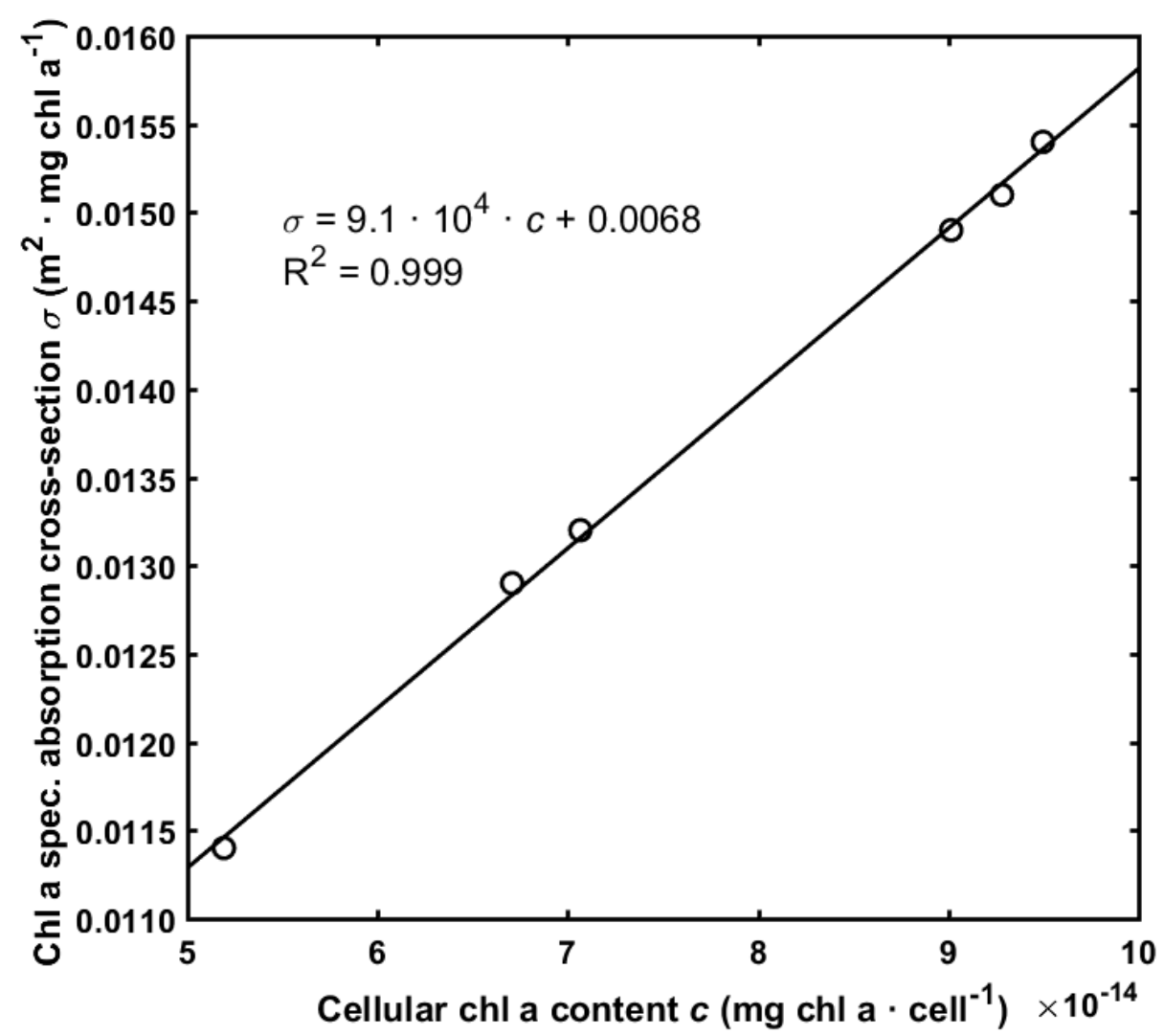

Figure 4.12: The cellular absorption cross-section $\xi$ relationship to cellular chlorophyll content per cell $c$. Linear fit equation and corresponding coefficient of determination $R^{2}$ are displayed in the figure, being the gathered parameters of Equation (4.5), $\xi_{\text {min }}=9.1 \cdot 10^{4} \mathrm{~m}^{2} \cdot \mathrm{mg} \mathrm{chl} \mathrm{a}{ }^{-2} \cdot$ cell and $m_{\xi}=0.0068 \mathrm{~m}^{2} \cdot \mathrm{mg} \mathrm{chl} \mathrm{a}^{-1}$. 


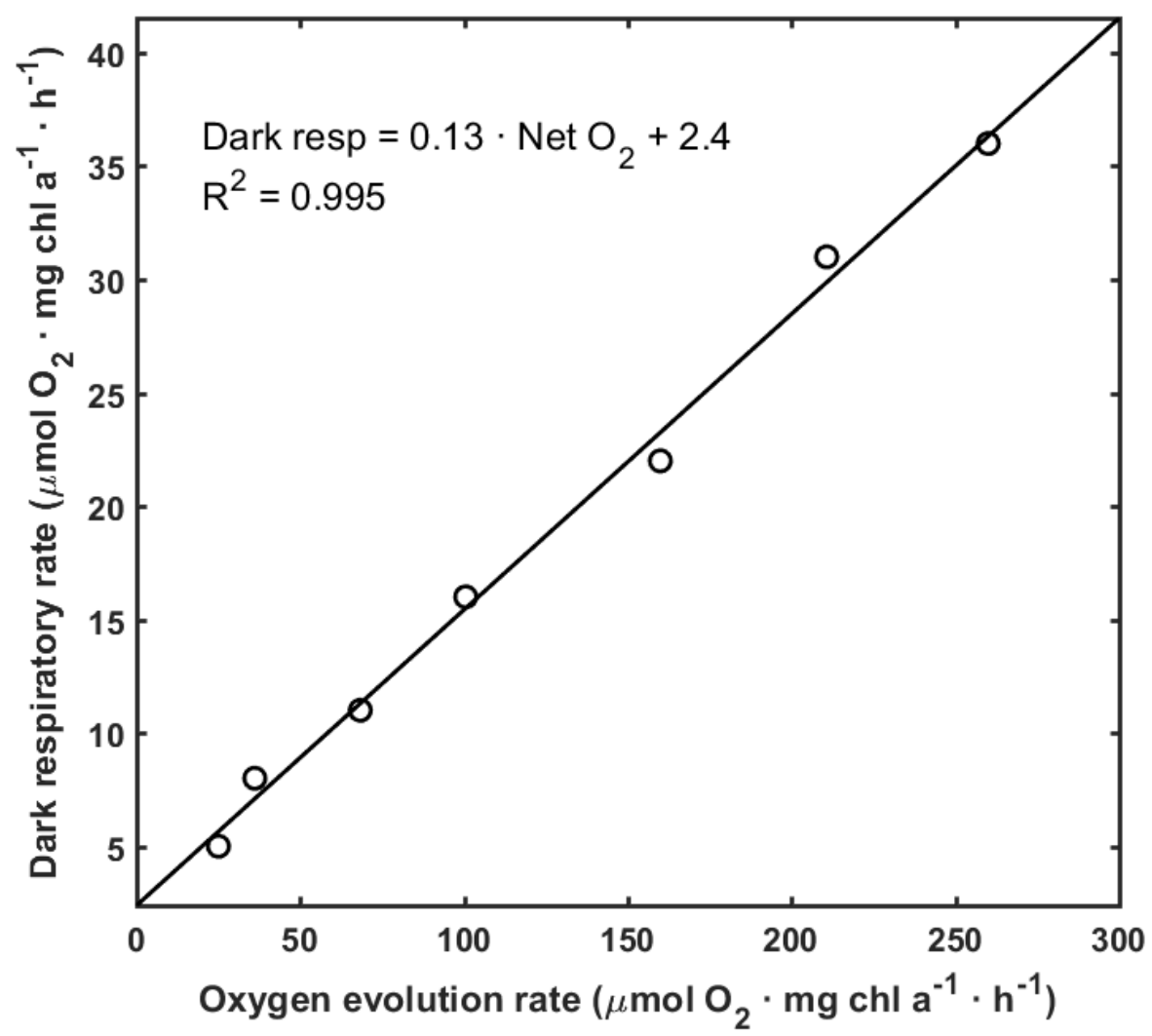

Figure 4.13: Relationship between dark respiration and photosynthetic yield for different light intensities. Linear fit equation and corresponding coefficient of determination $R^{2}$ are displayed in the figure, being the gathered parameters of Equation (4.14), $m=2.4 \mu \mathrm{mol} \mathrm{O}_{2} \cdot \mathrm{mg} \mathrm{chl} \mathrm{a}{ }^{-1} \cdot \mathrm{h}^{-1}$ and slope $\gamma=0.13$ for the studied light interval. 

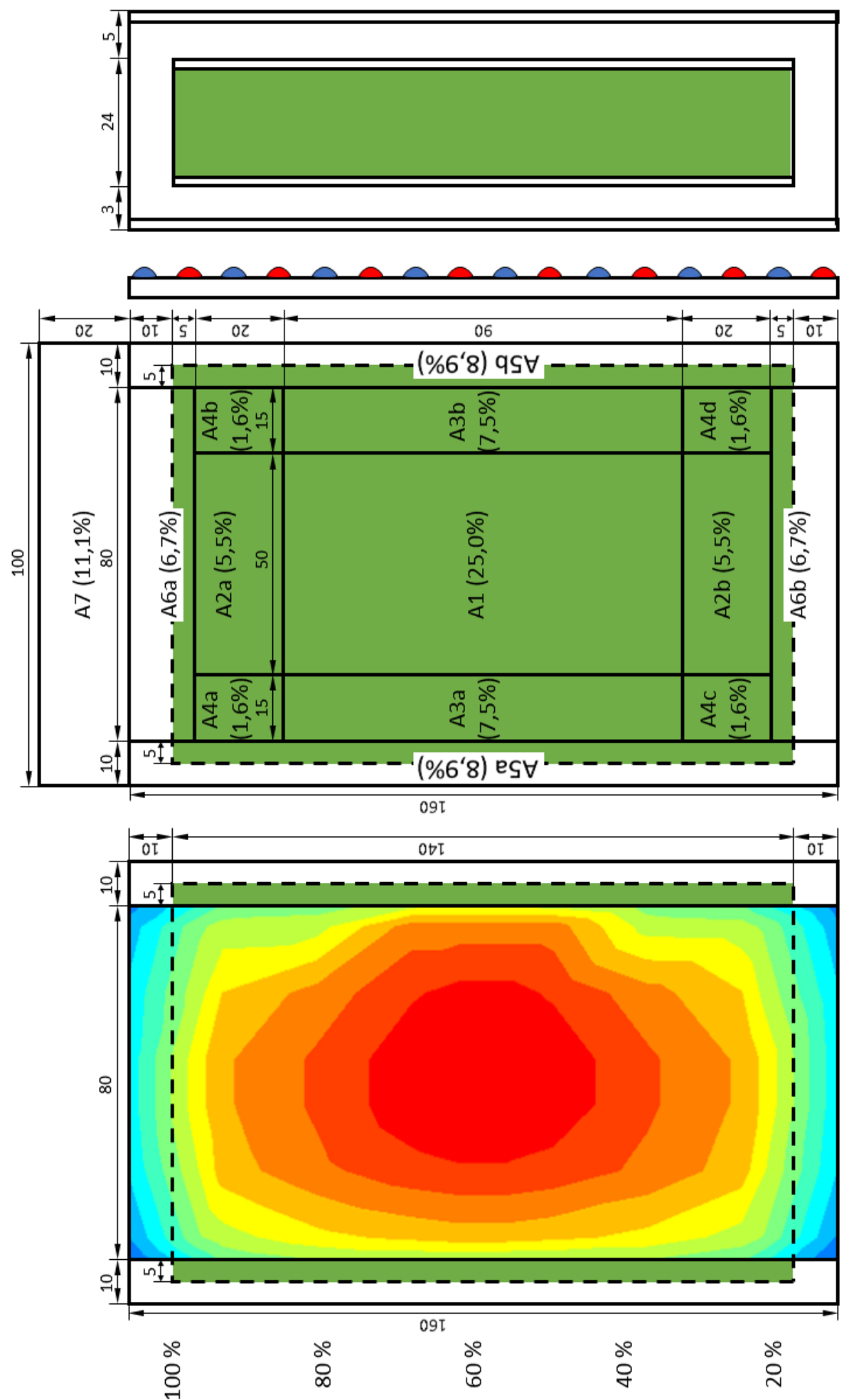
Chapter 4. Experimental characterisation of Synechocystis sp. PCC 


\section{5}

\section{Individual pigment}

contribution to overall in vivo

\section{absorption in Synechocystis sp. PCC 6803 cells}

Title: Individual pigment contribution to overall in vivo absorption in Synechocystis sp. PCC 6803 cells

Authors: David Fuente ${ }^{a, *}$, J. Alberto Conejero $^{b}$, Javier F. Urchueguía $^{a}$,

${ }^{a}$ Instituto de Aplicaciones de las Tecnologías de la Información y de las Comunicaciones Avanzadas, Universitat Politècnica de València, València, Spain

${ }^{b}$ Instituto Universitario de Matemática Pura y Aplicada, Universitat Politècnica de València, Valencia, Spain

* Corresponding author: dafueher@upv.es 


\section{Abstract}

Light absorption is a critical phenomenon in photosynthesis as light is its fundamental energy source. In this regard, unravelling the amount of radiation captured by each pigment would help in the understanding of photosynthetic processes that are directly controlled by the spectral characteristics of the light, such as non-photochemical quenching or state-transitions.

To do so, we reconstructed Synechocystis sp. PCC 6803 absorption signature from individual in vitro spectra of the principal photo-active pigments within this organism by first calculating the package effect factor within the photosynthetically active radiation range, by shifting the wavelength of pigment absorption spectra to match physiological behaviour and by finally summing the contribution of all these pigments. Moreover, reconstructing in vivo absorption spectra from individual chromophores allows a quantification of each pigment within the cells without having to apply spectrophotometric measurements, which is an indirect technique prone to error.

The estimated in vivo spectrum composed by the sum of the computed pigment signatures reproduces in a precise manner the experimental spectrum of the cell for all the studied wavelengths with the exception of the carotenoid band. Additionally, the gathered values of each pigment concentration are in agreement with typical values reported in literature. Further, we evaluated different illumination set-ups to assess the average absorption cross-section of the cells. It was concluded that cells exposed under orange and blue LEDs display a cross-section that almost doubles the value under broader white spectra or solar light. Finally, it is remarkable that exposure to halogen, warm white LED and orange monochromatic LED are suitable for stronger phycobilisome excitation.

Keywords: Absorption, pigment concentration, package effect, modelling, Synechocystis 


\subsection{Introduction}

\subsubsection{Light spectrum influence in photosynthesis}

Light is a principal factor regulating photosynthesis and influencing its global efficiency because light is the fundamental energy source for photosynthetic organisms and in many environments radiation is a limiting factor. The impact of light intensity on the photosynthesis apparatus and main related phenomena such as carbon fixation Sukenik et al. (1987), fluorescence Genty et al. (1989), electron flow distribution Miyake et al. (2005); Wilhelm and Selmar (2011) or photoinhibition Tyystjärvi and Aro (1996); Boehm et al. (2009), among others, has been widely researched and in silico quantified Eilers and Peeters (1988); Laisk et al. (2009); Westermark and Steuer (2016) since the second half of last century Rabinowitch and Govindjee (1969); Orr and Govindjee (2013). Nevertheless, the effect of the photon flux spectrum on photosynthesis has been less studied. In this regard, one can find scarce literature on colour effect experiments (de Mooij et al. (2016); Münzner and Voigt (1992); McCree (1971)) in comparison with the overwhelming quantity of published works on photosynthesis research, mostly dealing with white light or solar spectra experiments and just considering light intensity as a scalar magnitude Rabe and Benoit (1962). One of the reasons for this fact is that many researchers try to standardise their experiments by using constant white light and mostly care about the total intensity. However, white spectra can greatly differ in their photon flux distribution and therefore affect final conclusions of the trials or invalidate results benchmarking. Typical light sources employed in photosynthetic microorganism culture are plotted in Figure 5.1. Further, it is well-known that dissimilar spectral compositions do not just affect photosynthetic processes in which only excitons are directly involved, but also the different electronic pathways such as linear and cyclic electron routes Ma et al. (2007) and consequently the biomass formation of the cells too Kubín et al. (1983); Wang et al. (2016). In this regard, many cyanobacterial strains, such as Synechocystis, present longer dou- 
bling times Singh et al. (2009); Pollari et al. (2011) under pure blue light exposure and in some cases even no experimental growth is appreciable and cultures collapse Zavřel et al. (2015). For example, in cultures of cyanobacterium Arthrospira platensis, which is a species comparable to our model strain Synechocystis sp. PCC 6803, blue light led to the least biomass growth out of different light colours, but orange-red one promoted maximal growth rates Markou (2014). Yet, in other type of organisms owning other classes of pigments, this fact is not necessarily true.
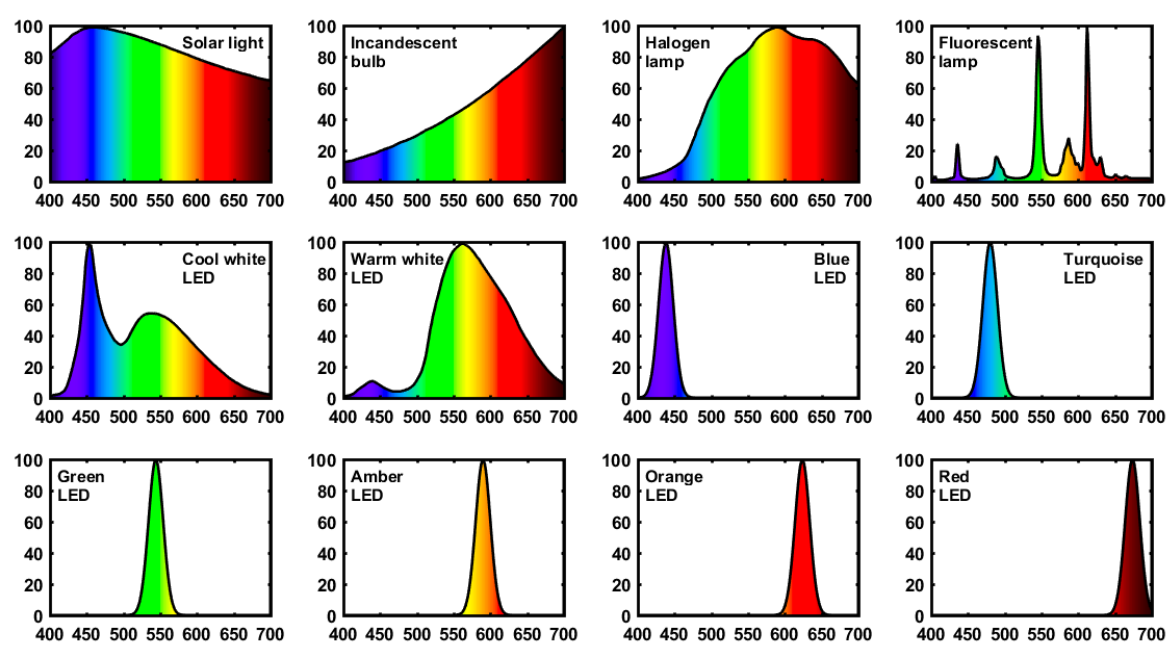

Figure 5.1: Photon flux spectra of common light sources for photosynthetic microorganisms cultures along the PAR range indicated in relative units. Photon flux distribution of each light source is depicted with the colours that belong to the corresponding wavelength.

\subsubsection{Absorption spectra of main pigments}

Light harvesting takes place inside the photo-active pigments present in the cell and are mostly located inside the thylakoid membrane in photosynthetic microorganisms, while hyperspectral confocal fluorescence microscopy technique indicates that non-assembled phycobilin proteins can be found in the cytoplasm, too Collins et al. (2012). The principal harvesting structures and their photosynthetic chromophores 
in Synechocystis are the PBS rods (phycocyanin), the PBS core (allophycocyanin), the PBS TE (allophycocyanin), carotenoids ( $\beta$-carotene, among others), PSII (chlorophyll a) and PSI (chlorophyll a). All of them own characteristic spectral signatures that altogether conform the overall cell absorption spectrum. Particularly, $\beta$-carotene is one of the most abundant carote-noids in cyanobacteria and the only carotenoid whose energy transfer capacity to the reaction centres has been already proofed Young and Frank (1996); Cerullo et al. (2002), though a higher quantum efficiency of the electron transport activities was found in the transfer between $\beta$-carotene and PSI than to PSII, where no light harvesting capacity has been reported Stamatakis et al. (2014). Though other main carotenoids (zeaxanthin, echinenone, myxoxanthophyll and $3^{\prime}$ - hydroxyechinenone) do also capture radiation, an effective energy transfer to the rest of the photosynthesis machinery has not been discovered yet.

On this subject, absorption spectra also depict the pigment concentration present in the cell and hence are another technique that helps understanding the physiological state of the organism. Indeed, exposure to high light Kopečná et al. (2012) or different light types Stramski and Morel (1990) modifies the overall absorption spectra due to photo-adaptive pigment composition variations. Additionally, such curves can be decomposed into single pigment spectra Wozniak et al. (2000). Though the in vivo absorption properties of chromophores do in general behave in a slightly different way in physiological conditions with respect to the extracts in organic solvents, their individual in vitro contribution can serve for the reconstruction of cell absorption spectra and yield acceptable results on the evaluation of each pigment concentration, even if the sum all individual signatures does not completely match the in vivo behaviour.

Previous investigations into absorption contribution can be found in literature, which basically follow two different strategies: either using solvent dissolved pigment signatures Bidigare et al. (1990); Bricaud et al. (2004) to construct the whole cell spectrum by summing them Bidigare et al. (1990); Ficek et al. (2004); Thrane et al. (2015) or de- 
convoluting the true in vivo absorption in different Gaussian curves Hoepffner and Sathyendranath (1991); Lohrenz et al. (2003).

\subsubsection{Pigment content assessment}

The calculation of each pigment concentration gives insight into the state of cells as these values respond to environmental and stress variations and are critical for a balanced light absorption. The common method to estimate the chlorophyll and carotenoid cellular concentration is to extract them with organic solvents and quantify their amount by means of the high-pressure liquid chromatography (HPLC) technique Ficek et al. (2004); Thrane et al. (2015). Alternatively, the measurement of the pigment content conforming the phycobilisomes is a challenging task: traditional methods cannot extract such pigments using organic solvents due to their hydrophilic affinity. These compounds get denatured when exposed to organic solvents MacColl (1983) and therefore they are commonly extracted in phosphate buffer solution at neutral $\mathrm{pH}$. Additionally, allophycocyanin and phycocyanin spectra overlap the chlorophyll absorption curve, even in aqueous extracts Sarada et al. (1999), and therefore a precise estimation of the true in vivo absorption coefficients of the phycobilin pigments stacked in APC and PC is rather complicated Kirk (1994). Furthermore, phycobilin proteins are normally estimated in a spectrophotometric way by means of empirical equations that incorporate absorption measurements at specific wavelengths Bennett and Bogorad (1973). However, even at these wavelengths the contribution of chlorophyll cannot be neglected since it can lead to a phycobilin quantification prone to error. Hence protocols have to be updated to account with such interference Lauceri et al. (2017). 


\subsection{Methodology}

\subsubsection{In vitro absorption spectra of photosynthetic pigments}

In the following, the procedure for reconstructing the in vivo absorption spectrum of Synechocystis cells from specific extinction coefficients (or analogously from absorption coefficients) of the respective spectra in organic solvents will be outlined. All employed in vitro absorption distributions are referenced in Table 5.1. Photosynthetic pigments are known to have shifted absorption spectra under electrical fields Liptay (1969). For example, the in vivo wavelength shift with respect to the in vitro situation is between 10-25 nm for the case of most carotenoids depending on the carotenoid and the employed solvent Kakitani et al. (1982). To start the reconstruction of the cell light harvesting, absorption curves of single pigments within organic solvents are needed. Such data imply not just the relative spectral distribution but also its absolute magnitude in $\mathrm{m}^{2} \cdot \mathrm{mg}^{-1}$ units. To do so, the extinction coefficient at the characteristic peak wavelength of the absorption signature is usually the only value reported. In our case, we chose data gathered with a reference solvent such as acetone due to its larger amount of available pigment data Wright et al. (1997). Henceforth, all pigment information is referenced to this solvent with the exception of echinenone extinction coefficient (petroleum ether) and the phycobiliproteins, whose absorption was measured from purified phycobilins and, particularly, from purified trimer complexes in the case of allophycocyanin MacColl (2004).

Though most of the spectra are well defined in previous works, there are few extinction coefficients that have to be hypothesised due to literature unavailability. In the case of the 3'-hydroxyechinenone molecule, which is the main chromophore of the orange carotenoid protein (OCP) protein Sedoud et al. (2014), the absorption signature of that protein has been used to this purpose Chábera et al. (2011). As we did not find estimates for its extinction coefficient, we imposed an identical one as the echinenone value. 
With respect of the phycobilin chromophores conforming the phycobilisomes, prior works suggest that the values at the peak wavelength in vivo for PC lie in the range 0.0040 to $0.0080 \mathrm{~m}^{2} \cdot \mathrm{mg}^{-1}$ Simis and Kauko (2012); Mishra et al. (2013), though for some phytoplankton species this number is reported to be below 0.0030 Yacobi et al. (2015). It has to be noted that there is an inversely proportional relationship between such protein concentration and the absorption coefficient. In the case of our strain, the concentration of phycocyanin is more than one order of magnitude higher with respect to the content in such species, hence an even lower coefficient is expected.

Moreover, limited data has been published regarding in vitro extinction coefficients of these chromophores. In Rakhimberdieva et al. (2001); Bryant et al. (1979) different molar extinction coefficients for both phycobilins are presented. The equivalent specific absorption coefficient for PC is roughly $0.0015-0.0017 \mathrm{~m}^{2} \cdot \mathrm{mg}^{-1}$, which is a much lower value than the previous referenced ones. To reconcile such magnitude uncertainty, we decided to assign an intermediate value for the phycocyanin mass absorption coefficient of 0.0025 and $0.0027 \mathrm{~m}^{2} \cdot \mathrm{mg}^{-1}$ for its allophycocyanin partner. The latter number arises from the molar extinction coefficient ratio of the protomer $(\alpha \beta)$ in each phycobiliprotein at their corresponding absorption peak Rakhimberdieva et al. (2001) and taking into account that the protomer weight in phycocyanin and allophycocyanin is 35.0 and $29.6 \mathrm{kDa}$, respectively Bryant et al. (1976).

Red chlorophylls contribution was neglected as there are only 4-6 molecules per PSI complex in cyanobacteria Gobets and van Grondelle (2001), its absorption is mostly located in the infra-red band, i.e. outside the PAR radiation and this chlorophyll absorption in Synechocystis is relatively small in comparison with other cyanobacterial species Gobets et al. (2003).

\subsubsection{Estimation of the package effect}

As already stated, the sum of single in vitro absorption signatures, represented by the absorption coefficients at any wavelength, do not al- 
ways coincide with in vivo spectra. In this context, pigment absorption coefficients under physiological conditions cannot be considered as unequivocal magnitudes, as light capture in the chromophores depends on the cell size, geometry, the type and the amount of harvesting compounds Morel and Bricaud (1981a); Bricaud and Stramski (1990); Mitchell and Kiefer (1988), phenomenon called as package effect Kirk $(1975 a, b)$. In this regard, pigment coefficients inside living cells are always lower than the ones measured in dispersed homogeneous solutions, also referred to as unpacked coefficients. This absorption reduction is set by the dimensionless factor $\mathrm{Q}_{\mathrm{a}}^{*}$ representing the package effect:

$$
Q_{a}^{*}(\lambda)=\frac{a^{*}(\lambda)}{a_{\text {sol }}(\lambda)}
$$

where $\mathrm{a}^{*}$ is the true absorption coefficient and $\mathrm{a}_{\mathrm{sol}}$ the one corresponding to the ideally dispersed pigment solution.

In order to reconstruct the true in vivo cell absorption spectrum from in vitro coefficients, and in this way calculate all pigment concentrations, the package effect has to be determined. Such magnitude can be estimated by means of the theoretical framework proposed by Morel et al. Morel and Bricaud (1981a):

$$
Q_{a}^{*}\left(\rho^{\prime}\right)=\frac{3}{2} \frac{Q_{a}\left(\rho^{\prime}\right)}{\rho^{\prime}}
$$

Hence, the dimensionless factor $\rho^{\prime}$, which rules the discreteness of absorption, must be previously calculated. To do so, homogeneity and sphericity of the cells have to be assumed and in this regard most of Synechocystis photo-active pigments are located in the plasma and thylakoid membranes Zhang et al. (2015). In addition, the latter layers are structured as spherical membranes within the cell, similarly to "matryoshka dolls" following an intra-laminar arrangement Liberton et al. (2006). Therefore, the homogeneity assumption seems plausible in this strain. Additionally, previous works have already shown satisfactory results when assuming a spherical approximation for spheroidal cells Bricaud et al. (1988); Nelson and Prezelin (1990). 
The experimental absorption efficiency, knowing the measured in vivo absorption spectrum, is given at each wavelength $\lambda$ by:

$$
Q_{a}(\lambda)=\frac{2}{3} a^{*}(\lambda) c_{i} d_{i}
$$

being $c_{i}$ the intracellular chlorophyll concentration and $d_{i}$ the cell internal diameter. This is valid for the case of equally-sized particles of diameter $d_{i}$. However, at culture scale it is common to find a population of cells with different diameters. This is the so called polydispersion and its effect is taken into account through the size distribution function $\mathrm{F}(\mathrm{d})$. Mathematically, $\mathrm{F}(\mathrm{d})$ is treated as a weight function, so the efficiency factor will be weighted as follows Bricaud and Morel (1986):

$$
\bar{Q}_{a}(\rho)=\frac{\int_{0}^{\infty} Q_{a}(\rho) F(\rho) \rho^{2} d \rho}{\int_{0}^{\infty} F(\rho) \rho^{2} d \rho}
$$

Then the anomalous diffraction approximation, which was described by Van de Hulst Van de Hulst (1957), and is a particular case of the Mie-Lorentz theory, will be leveraged. The approximation is based on the next assumptions: the size of the particles is one order of magnitude larger than the wavelength, hence the corresponding parameter $\alpha$, defined in Equation (5.7), is greater than 10 and the particles are weakly absorbing and therefore the imaginary part of the refractive index $\mathrm{n}^{\prime}$ is close to zero. Thanks to these assumptions, a simple analytic function of $\mathrm{Q}_{\mathrm{a}}$ is available. Once the efficiency magnitude has been computed via Equations (5.3) and (5.4), the relationship (5.5) can be solved for $\rho^{\prime}$ at each wavelength:

$$
Q_{a}\left(\rho^{\prime}\right)=1+2 \frac{e^{-\rho^{\prime}}}{\rho^{\prime}}+2 \frac{e^{-\rho^{\prime}}-1}{\rho^{\prime 2}}
$$

Note that $\rho^{\prime}$ and $\lambda$ are linked via Equations (5.6) and (5.7). Afterwards, we can determine the imaginary part of the refractive index $n^{\prime}$, taking into consideration following relationships:

$$
n^{\prime}=\frac{\rho^{\prime}}{4 \alpha}
$$




$$
\alpha=\frac{\pi d_{i}}{\lambda}
$$

By doing so, we will able to check out that at any wavelength $\lambda$ this index is close to zero as initially postulated. Finally, the package effect can be computed through the Equation (5.2).

Knowing the pigment concentrations in the cell, their in vitro absorption properties and the package contribution allows the reconstruction of the in vivo absorption spectrum. In our case, we want to proceed in the opposite direction, we aim at predicting light harvesting composition departing from the cell absorption properties.

\subsubsection{Assessment of the cellular pigment concentration}

Once the package effect has been calculated for each wavelength, it can be applied to the sum of all light harvesting spectra to fit in vivo absorption curve. In this way, a rough prediction of each pigment content is available. To this purpose, we manually fitted the resulting curve by shifting the spectra to match the absorption peaks of the cell and in this manner the amount of all pigments may be approximately predicted. With the exception of the carotenoids absorption band, it is straightforward to elucidate the required shift to correctly reconstruct the cell absorption spectrum. Additionally, the knowledge of the absorption coefficients of all light harvesting molecules facilitates the determination of every pigment concentration as there are always narrow intervals of the spectra were one chromophore is the main contributor to absorption and therefore a proper fit of such part of the spectrum is feasible. For chlorophyll a, the blue range area was positively displaced $8 \mathrm{~nm}$, whereas for the red one, $12 \mathrm{~nm}$ as done in Bidigare et al. (1990).

Yet, blue-green absorption results from the combination of at least five different carotenoids whose absorption signatures overlap themselves without displaying any differentiated peaks in vivo in normal growth conditions. However, for some mutant strains and extreme environmental conditions, carotenoid expression can be largely promoted and 
corresponding absorption peaks can be distinguished when cellular absorption is measured. This is the case for Synechocystis strains under nitrogen starvation von Wobeser et al. (2011), as under such conditions minor absorption peak appear at 485 and $520 \mathrm{~nm}$, which correspond to the presence of $\beta$-carotene, zeaxanthin molecules and myxoxanthophyll, respectively. Such phenomenon aids one to shift the wavelength of in vitro spectra to converge with the in vivo related curves. All displacements are shown in Table 5.1.

To simplify the reconstruction of the absorption spectrum for the carotenoids band, several assumptions were taken: First, $\beta$-carotene and zeaxanthin share practically identical in vitro signatures with a slightly higher specific extinction coefficient of the carotene, hence we simply imposed a mass ratio of 1.5 , which is the mean value calculated from prior literature. Second, due to the marginal contribution to absorption by $3^{\prime}$ - hydroxyechinenone molecules, we assumed a concentration equal to the only experimental value reported in literature Takaichi et al. (2001).

\subsubsection{Light absorption by each in vivo absorption spectra}

The calculation of the number of quanta absorbed by each pigment is achieved by means of the individual characteristic in vivo absorption spectra of each chromophore, also referred to as absorption coefficients, as these represent the probability distribution of absorbing light at any wavelength under a given light source since they are an inherent optical property. Particularly, such spectra can be averaged into a mean value by the photon flux density (PFD) of a given light source, such as solar light, light-emitting diode (LED) lamps or any other type of artificial light source. Thus, using the in vivo absorption spectra of the corresponding pigment and considering the light source employed in 
the experiment, the absorption cross-section $\sigma$ is computed by means of the Equation (5.8):

$$
\sigma_{A, P F D}=\sum_{\lambda=400}^{700} a(\lambda) \cdot \operatorname{PFD}(\lambda)
$$

where $\sigma_{A, P F D}$ denotes the absorption cross-section of the photo-active chromophore $A$ under a light source represented by its PFD. However, not just lamp emission spectra can be used for this purpose but also remaining photon flux distributions along the PBR. This includes the light spectrum predominating at deeper distances inside cell cultures with respect to the light source, which is basically composed of green wavelengths for the case of white radiation due to selective absorption by biological suspensions.

With respect to the amount of excitation that could be in principle transmitted to each PS type, it has been assumed the same absorption capacity for molecules linked to one or the other photosystem. Regarding the parameters needed to assess such feature, it has to be noted that most of the cell chlorophyll content is associated with PSI since this comlly, PSI:PSII ratios lie in the range 4-6 in this strain Shen et al. (1993); Tian et al. (2011); Moal and Lagoutte (2012). Therefore, the proportion of the chlorophyll a assuming a PSI:PSII ratio of 5 and a number of chlorophyll molecules of 96 and 35, respectively, is that $90 \%$ are chla $a_{P S I}$ and the rest chla $a_{P S I}$, whereas $92 \%$ of APC protomers are located in the APC core and rest in the TE van Stokkum et al. (2018). Besides, the number of molecules of $\beta$-carotene in each photosystem is 22 in PSI Jordan et al. (2001) and 11 in PSII Umena et al. (2011), respectively.

With the exception of $\beta$-carotene, the rest of carotenoids have been proven to own auxiliary roles such as photo-oxidative damage prevention and photosystem assembly, among others, but only the former carotenoid seems to have excitation transfer routes to chlorophyll, yet with low efficiency, especially to PSII Telfer (2002). Therefore, only the carotene has been modelled regarding light absorption contribution. 


\subsection{Results and discussion}

\subsubsection{Package effect calculation}

In order to assess the package effect, several cell properties are necessary. Such quantities, which will be described in the following, were gathered in the growth and optical conditions outlined in a previous work Fuente et al. (2017). First, the measured mean external diameter of the cell $d$ is $2.3 \mu \mathrm{m}$ and the chlorophyll mass per cell $c$ accounts for $30 \mathrm{fg}$. Assuming as in Moal and Lagoutte (2012) a periplasmic space diameter of $0.4 \mu \mathrm{m}$ and a similar cell size distribution, the internal diameter $\mathrm{d}_{\mathrm{i}}$ is supposed to be $1.9 \mu \mathrm{m}$ and the intracellular chlorophyll concentration $\mathrm{c}_{\mathrm{i}}$ amounts to $8.8 \times 10^{6} \mathrm{mg} \mathrm{chl} \mathrm{a} \cdot \mathrm{m}^{-3}$.

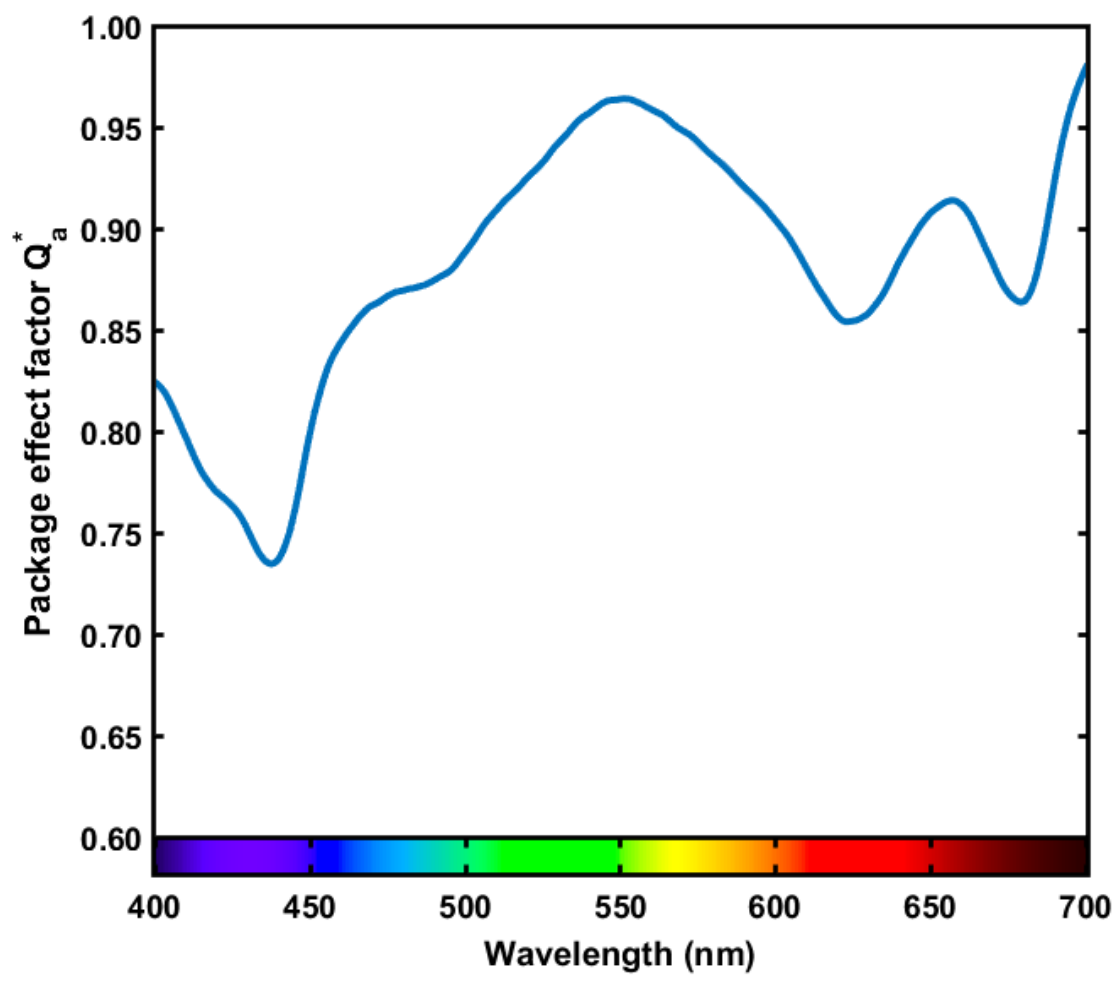

Figure 5.2: Computed package effect factor $\mathrm{Q}_{\mathrm{a}}^{*}$ for Synechocystis WT along the PAR range. 
Regarding the validity of the package effect calculation, it has to be noted that the predicted imaginary part of the refractive index $\mathrm{n}^{\prime}$ is almost zero along the whole PAR range as expected (data not showed). Moreover, this index and the package effect itself $\mathrm{Q}_{\mathrm{a}}^{*}$ (Figure 5.2) are in line with the corresponding magnitudes in many other species Bricaud et al. (1988). Our calculated package effect factor at $675 \mathrm{~nm}$ is 0.87 and concurs well with the expected value for the assumed cell diameter and chlorophyll a concentration Nelson and Prezelin (1990).

\subsubsection{Pigment quantification through the reconstruction of the in vivo absorption spectrum}

Reconstructed in vivo absorption spectrum is shown in Figure 5.3. Three main group of pigments are depicted: on the one hand chlorophyll a with its characteristic peaks, both phycobilins absorbing yellow to red radiation and the main carotenoids, whose absorption is located in the blue-green range. First, it is noteworthy to remark that the calculated in vivo spectrum matches the experimental one with the exception of the carotenoids waveband. Apart from this, the most striking result to emerge from the reconstruction is that we are able to predict within a reasonable precision the concentration of the main light harvesting compounds present in the cell, while gathering an absorption spectrum that is very close to the experimental signature. The in silico prediction for chlorophyll a content is $1,800 \mathrm{mg} \mathrm{chl} \mathrm{a} \cdot \mathrm{m}^{-3}$, just $9 \%$ higher than our measured value (Table 5.1).

The prediction for the carotenoid content also lies within the range of minimal and maximal values reported in previous research carried out on this strain. In all those works, Synechocystis was grown at buffered $\mathrm{pH}$ around 7.5 and $30{ }^{\circ} \mathrm{C}$ as in our case, yet the irradiance was smaller in all works. This could be one reason for the slightly overestimation of the carotenoids concentration, yet an incomplete pigment extraction in that published works cannot be surely discarded. Anyhow, the different experimental set-ups, i.e. cell densities, growth conditions and the 
geometry of the reactor, just allow a qualitative benchmarking between the experimental and our in silico determination.

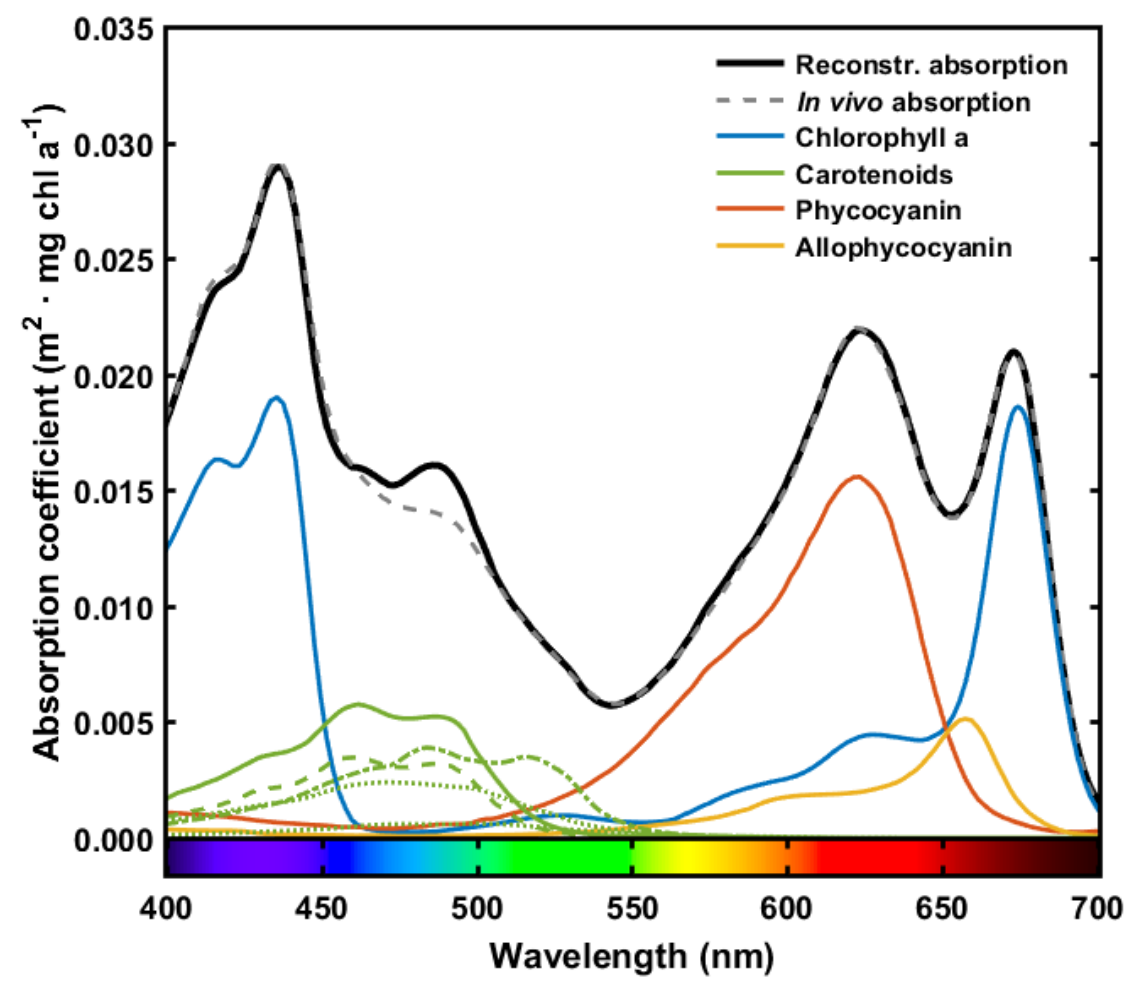

Figure 5.3: Reconstructed Synechocystis absorption spectrum along the PAR range of cells acclimatised to a total intensity of $100 \mu \mathrm{mol}$ photons $\cdot \mathrm{m}^{-2} \cdot \mathrm{s}^{-1}$ of cool white LED light. Black signature (-) corresponds to in vivo spectrum, while grey curve (-- ) is the reconstructed absorption. Computed in vivo absorption contribution of each pigment is also depicted: chlorophyll a (-), carotenoids (-) [ordered with respect to their maximum absorption peak, $\beta$-carotene (-), myxoxanthophyll (-.), zeaxanthin (--), echinenone (...) and $3^{\prime}$ - hydroxyechinenone (...)], allophycocyanin (-) and phycocyanin (-). 3' - hydroxyechinenone spectrum can be distinguished from echinenone's one since the former one is smaller.

Regarding phycobiliproteins, we could only found two contributions where such proteins were quantitatively determined in Synechocystis WT. For PC, both works Tsunoyama et al. (2009); Touloupakis et al. (2015) report values between 6,500 and 7,000 $\mathrm{mg} \cdot \mathrm{mg} \mathrm{chl} \mathrm{a}^{-1}$ at a similar chlorophyll concentration, whereas for APC concentrations below 
2,000 $\mathrm{mg} \cdot \mathrm{mg} \mathrm{chl} \mathrm{a}{ }^{-1}$. Such values are relatively close to our hypothesised amounts (Table 5.1). On this subject, the computed PC/APC mass ratio of 3.4 is in line with a previous experimental value of 3.6 Tsunoyama et al. (2009) and consistent with the stoichiometric mass proportion between both phycobiliproteins, i. e. for a total amount of 12 APC core-trimers $(\alpha \beta)_{3}$ and 18 PC rod-hexamers $(\alpha \beta)_{6}$ Adir (2005), the latter add up to an equivalent mass of 36 trimers for the whole PC antenna, that is thrice the core mass. This number together with the fact that just few percent of all rods are directly attached to the thylakoid membrane Kondo et al. (2007) is in alignment with our modelling estimation. Moreover, knowing the total mass of the PBS core, it is possible to approximately determine the number of PBS in the cell. The measured cellular chlorophyll a content $c$ is $30 \mathrm{fg}$. This figure together with the assumption of a photosystem I/II ratio of 5, leads to roughly 50 PBS per 100 PSII monomers, estimation also consistent with a previous work on this strain, suggesting that there are 44 PBS per 100 PSII monomers Moal and Lagoutte (2012).

Regarding the carotenoids contribution, it is important to highlight the difficulty of a perfect reconstruction of the in vivo absorption behaviour from single in vitro experiments in solvents. Indeed, the cell extract absorption signature in an organic solvent presents several local minimamaxima in the blue-green band due to different carotenoids and chlorophyll absorption peaks that are absent in the in vivo curve Kilian et al. (2007). Though the difficulty of an exact determination of each carotenoid concentration for the reasons already detailed above, the calculated amounts are in agreement with reported values (Table 5.1).

Further, it is possible to estimate the proportion of the light captured by the phycobilisomes as depicted in Figure 5.4. This is another remarkable feature since PBS can deliver excitation energy in a more or less balanced manner towards both photosystems, while chlorophyll aexcitation can be hardly transferred between photosystem I and II, principally through spill-over energy mechanisms, which have limited range and only occur during some type of state-transitions processes. In summary, yellow to red light seems to be optimal for photosynthesis per- 


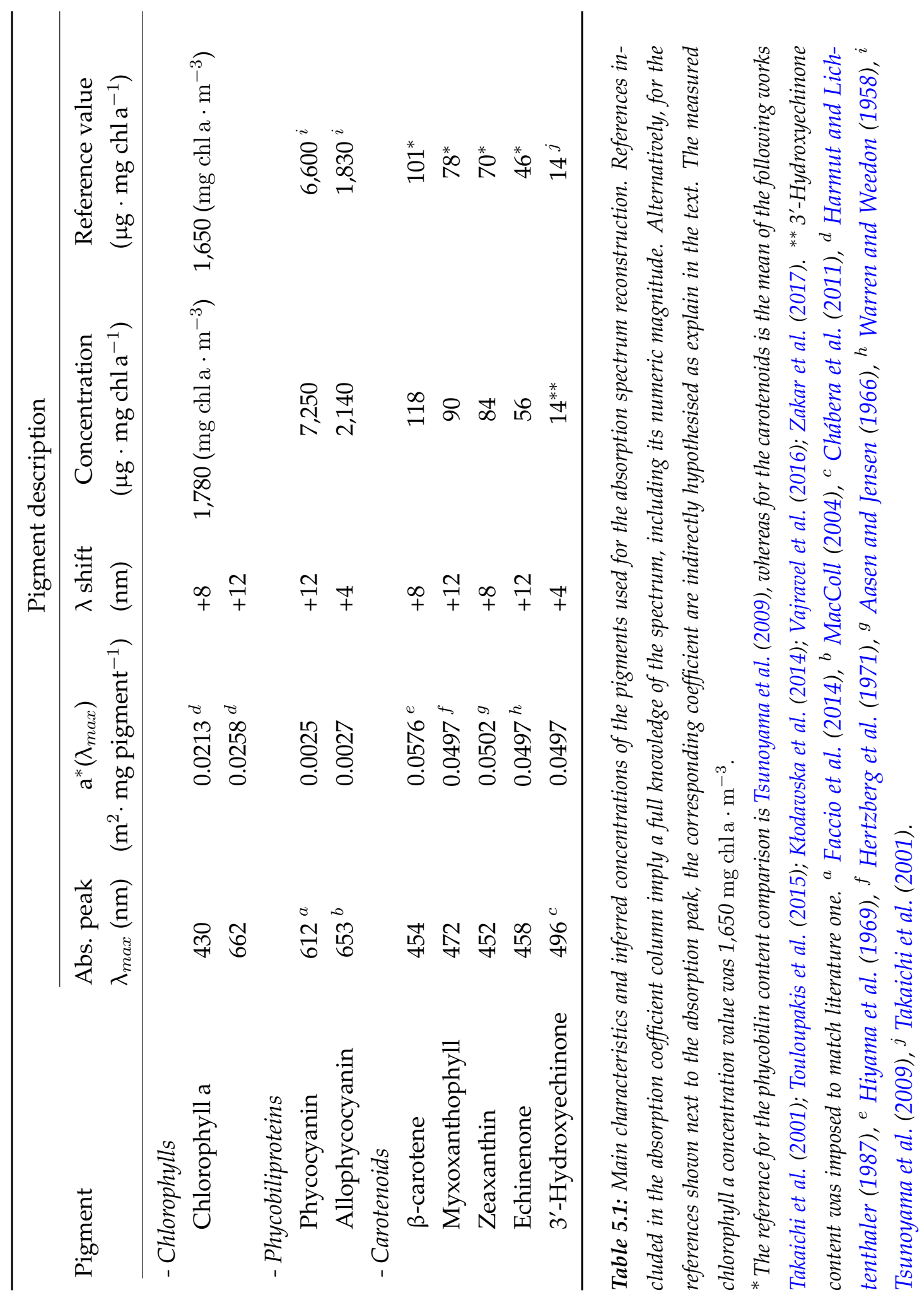




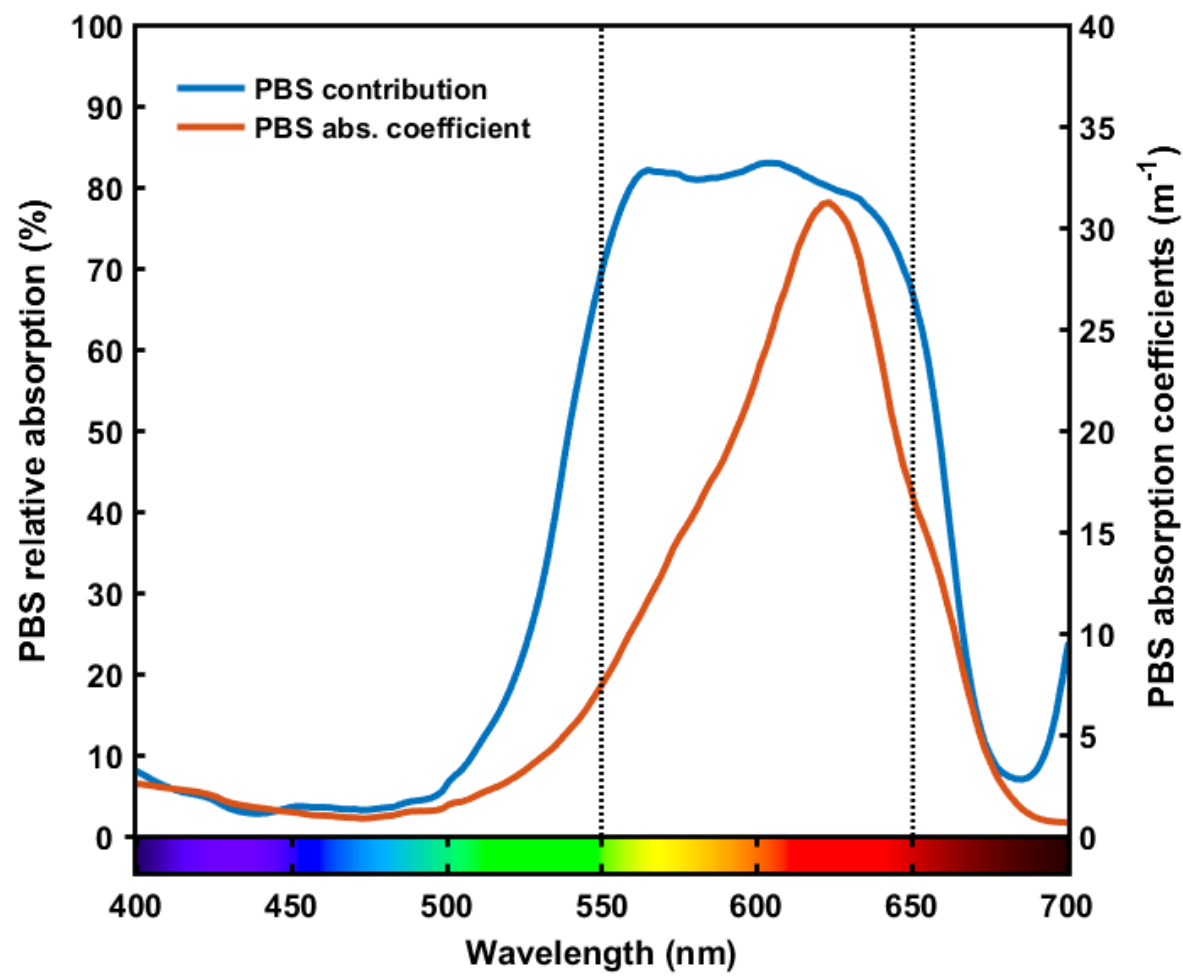

Figure 5.4: Phycobilisomes absorptive spectral characteristics of Synechocystis cells acclimatised to a total intensity of $100 \mu \mathrm{mol}$ photons $\cdot \mathrm{m}^{-2} \cdot \mathrm{s}^{-1}$ of cool white LED light. PBS absorption contribution with respect to the cellular one (-) and total PBS absorption coefficients (-) expressed in $\mathrm{m}^{-1}$ units are also depicted. Dashed vertical lines approximately indicate the wavelength range (550-650 $\mathrm{nm}$ ) in which PBS absorption rules over chlorophyll a one.

formance. However, it is known that yellow light does not promote an electron flow as high as the one arising under orange light Tyystjärvi et al. (2002). This could be in principle attributable to a minor but necessary excitation of chlorophyll amolecules in PSI, which does not occur under yellow photons. Yet, further investigation is required to shed light on this matter and by doing so assess the optimal wavelength combination for photosynthesis in cyanobacterial cells. 


\subsubsection{Absorption cross-section under different illumination environments}

Knowing the individual absorption of each pigment, one can estimate the light harvested by each chromophore. Indeed, this is important to elucidate where the light is entering the cell as such distribution will impact to some extent the rest of photosynthesis processes. In this regard, cellular absorption cross-section split into each pigment crosssection for each light source is depicted in Figure 5.5. The main outcome of this plot is that for the so called white lamps, which own a broad and white emission spectrum, and for the solar illumination as well, the absorption cross-section for the whole PAR range is around 0.010-0.012 $\mathrm{m}^{2} \cdot \mathrm{mg} \mathrm{chl} \mathrm{a}^{-1}$, while for monochromatic LED lamps dissimilar results are obtained: there is a much lower absorption for some LED lamps, while the opposite happens for other colour cases. Particularly, under blue and orange light Synechocystis absorption doubles the light harvesting for cells exposed to the previous white spectra, since the absorption peaks practically overlap the LED emission ones. Remarkably, for blue and red light most of the radiation is captured by chlorophyll apresent in PSI units, whereas for orange radiation peripheral PBS rods are responsible for the majority of the light capture.

In theory, the latter illumination set-up should be the ideal one for Synechocystis cells due to the higher flexibility of PBS antennas to distribute excitation energy among the photosystems by means of antenna movement on the thylakoid membrane surface, while excessively excited PSI can hardly transmit such energy to PSII through the reverse spill-over mechanism.

\subsection{Conclusions and future work}

In this research, we showed that it is feasible to reconstruct in vivo absorption signature of a cyanobacterial cell by correcting that spectrum with the corresponding package effect factor. Only carotenoid band 
was not precisely estimated, i. e. the application of the package effect is not sufficient to explain the behaviour of this type of chromophores under physiological conditions. One reason behind such dissimilarity with respect to the absorption inside an organic solvent could be that the electronic properties may vary when these compounds are attached to other protein molecules. Moreover, not all carotenoids have a light harvesting role in the cell. Nevertheless, the reconstruction process satisfactorily reproduces the in vivo signature and hence as a direct consequence of it, each pigment concentration can be gathered within a reasonable accuracy as suggested by own and literature available data for Synechocystis sp. PCC 6803 strain. Alternatively, we showed the relative participation of each chromophore in total light capture and quantitatively described the radiation harvested for any of the usual light sources employed in cultures grown in PBRs. As expected, under some monochromatic LED lamps cells are capable of absorbing many more photons. Such numbers have to be taken carefully into consideration then a more assessment is required when estimating the optimal lamp as dense cultures may collapse overall productivity by harvesting light above their photosynthesis rates capacities and thus, wasting available energy for layers of cells located further from the light origin. Future research will cope with the dynamic evolution of pigment composition due to photo-adaptive changes since the knowing the absorption coefficients would allow the quantification of the chromophore at any moment of time. Finally, the outlined modelling framework has been already use for marine optic applications, yet there is no work, to the best of our knowledge, applying such approach to unravel pigment contribution to light harvesting in photobioreactor cultures and thus to the photosynthesis processes for biotechnological applications. In fact, the here proposed methodology can be coupled to a photosynthesis model which incorporates photons as the input for the excitation energy transfer among chromophores as a first step to later transform them into effective electron flow. Hence, understanding the origin of the excitation arisen in the cell would shed light on the fate of such energy and so, how light characteristics affect the physiology of the organism. 


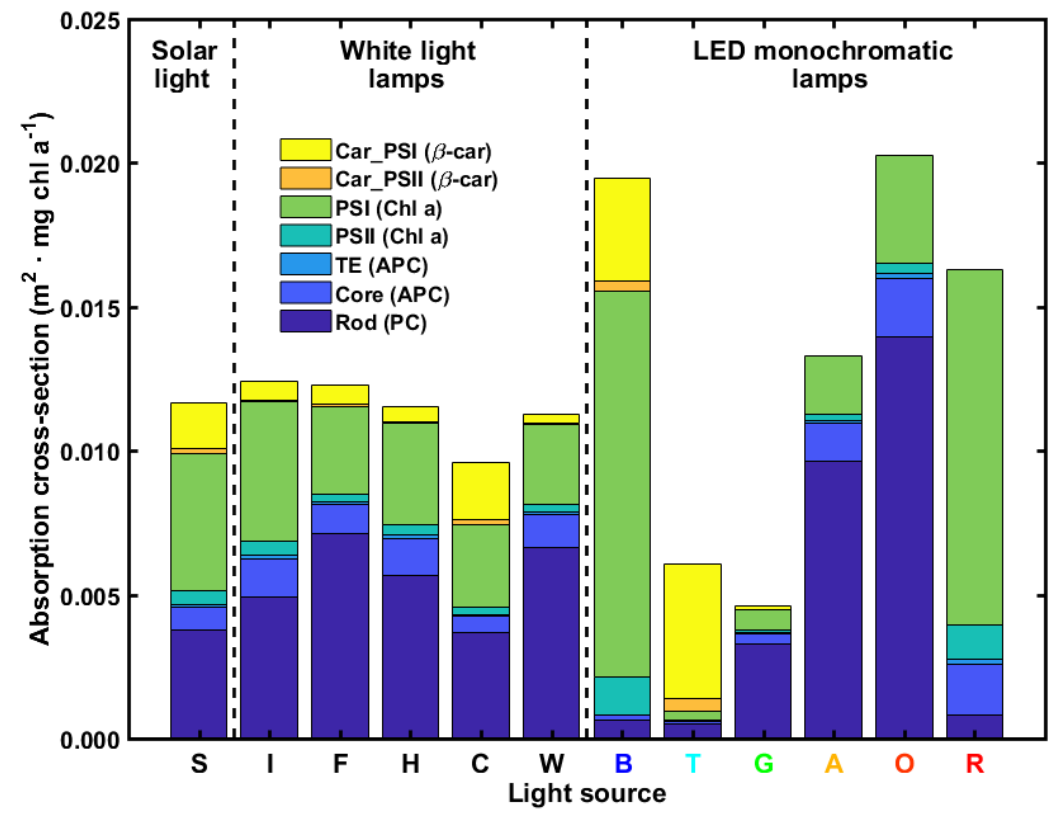

Figure 5.5: Absorption cross-section of individual pigments for Synechocystis cells acclimatised at $X$ intensity under different illumination set-ups. Pigment absorption is partitioned in the main light harvesting structures present in the cells (including their chromophores): PBS rod (PC $\square$ ), PBS core (APC $\square$ ) and PBS TE (APC $\square$ ), PSII (Chl. $a \square)$, PSI (Chl. $a \square)$, light harvesting carotenoid of PSII ( $\beta$-car. $\square)$, and the one for PSI: ( $\beta$-car. $\square)$. The employed light sources with their corresponding emission spectra are the ones shown in Figure 5.1. These sources can be divided into solar light (S-sun), white light lamps with a broad spectrum (I-incandescent bulb, Ffluorescent lamp, H-halogen lamp), C-cool white LED and W-warm white LED) and monochromatic LEDs. In the last case, they present a Gaussian shape, whose peak is located for each lamp at the following wavelengths in $n$ m, including the colour of the emission peak wavelength): B-blue 438, T-turquoise 480, G-green 544, A-amber 590, O-orange 624, R-red 674. 


\section{6}

\section{General discussion of the}

results

\subsection{Article "Light distribution and spectral composition within cultures of micro-algae: Quantitative modelling of the light field in photobioreactors"}

In the following, a summarised discussion of the published article entitled "Light distribution and spectral composition within cultures of microalgae: Quantitative modelling of the light field in photobioreactors" will be outlined. Figure 2.1 depicts in a simplified manner the proposed modelling scheme for estimating the light field. In this regard, attenuation coefficients for downward irradiance can be estimated by means of Kirk's semi-empirical correlation (Equation (2.6)), which uses absorption and scattering coefficients for assessing the PAR attenuation coefficients. These IOPs are shown in Figure 2.4, while computed attenuation coefficients are displayed in Figure 2.6. These coefficients resemble the absorption signature since the mayor contribution to the attenuation is due to absorption in the assessed strains, as it can be appreciated in 
Figure 2.5, were only scattering seems to have a more important role for green wavelengths. Attenuation coefficients, given in chlorophyll concentration units, seem to be constant for the studied incident light intensities. This is probably because the analysed irradiance values are not sufficient to reach photoinhibition. Besides, though such graphs remain approximately constant, the attenuation coefficients drop as light intensity increases, especially in the most intense range of radiation. The reason behind is that chlorophyll content is inversely proportional to irradiance (Figure 4.5), leading to decreasing coefficients, given not per chlorophyll a basis but as total coefficients in $\mathrm{m}^{-1}$, as depicted in Figure 4.10. The next step is to in silico calculate the mean downward attenuation coefficient valid for the whole PAR interval. To this purpose, the developed AFA algorithm was employed, which requires as additional input the lamp emission signature, the PBR length and the cell density. The algorithm first computes the PAR averaged attenuation coefficient by means of equation (2.12) and thereafter the mean irradiance within the culture through equation (2.13). As both magnitudes rely on each other, a computation loop is required to solve this system of equations. Besides, it is remarkably that the convergence of the process is achieved in within just two or three iterations. After the coefficient is gathered, one can calculate the light decay via the Lambert-Beer law, as indicated in equation (2.7). In this case, only the PAR exposure irradiance is needed. Alternatively, it has to be noted that exposure and acclimation environments can be the same and that as long as the exposure intensity is not enough long to promote pigment composition changes, one can admit that the optical properties of the cells remain the once that derive from the previous long-term acclimation environment. Finally, it is also possible to estimate how the light is attenuated spectrally. To do so, we simply need to utilise the originally computed attenuation coefficients shown in Figure 2.6 and input a photon flux distribution of the light source to the Lambert-Beer law as outlined in equation (2.5). In summary, through the described methodology, it is possible to estimate the light distribution field as PAR averaged intensities ( $\mu \mathrm{mol}$ photons $\cdot \mathrm{m}^{-2} \cdot \mathrm{s}^{-1}$ ) or in terms of photon flux density $\left(\mu \mathrm{mol}\right.$ photons $\cdot \mathrm{m}^{-2} \cdot \mathrm{s}^{-1} \cdot \mathrm{nm}^{-1}$ ). 
Once the PAR averaged attenuation coefficients were calculated, the predictions were compared with experimental data for both strains. The results, plotted in Figure 2.7, suggest that the model predicts within a reasonable accuracy the light attenuation in both strains and for five different cell densities under white LED light. Though in some cases, the prediction is not as accurate as in others, we hypothesise that the main cause for such an uncertainty is the fact that we don't exactly know the average acclimation intensity for the cells, as these experimental data were taken from another research group. Similarly, we performed the same benchmark with different exposure lamps, namely white but also blue, green and red LED lamps, obtaining satisfactory results again. Interestingly, attenuation for blue monochromatic light was similar in both strains, whereas for red attenuation in wild-type cultures is much pronounced in comparison with Olive mutant. The reason behind is for such dissimilarity is that the main phycobilin pigment absorbing red quanta is missing in the latter strain. Regarding attenuation in spectral terms, we performed different simulations, as shown in Figure 2.9 and 2.10. The most remarkable outcome of the last plot is that after only $2 \mathrm{~cm}$ of optical path-length the white led spectrum has been gradually transformed into almost pure green light, fact that has many implications for biotechnological applications of dense photosynthetic cultures. Finally, we performed the a simulation calculating the theoretical attenuation coefficient for cultures at different cell densities and acclimation intensities for both strains. For the sake of example, attenuation coefficients for downward irradiance at ultra-dense cell-densities of $25,000 \mathrm{mg} \mathrm{chl} \mathrm{a} \cdot \mathrm{m}^{-3}$ account for around $250 \mathrm{~m}^{-1}$ in the case of wild-type and $150 \mathrm{~m}^{-1}$ for Olive strain and there are wide range of white light intensities.

For turbid waters, such as high cell-density suspensions, the ratio between the attenuation coefficient for downward irradiance $\mathrm{K}_{d}$ and the one for scalar irradiance $\mathrm{K}_{0}$, is ranged between 1.01 and 1.06 for organisms whose scattering to absorption quotient $b / a$ lies is the interval 0.3 to 30 Kirk (1994). In our particular strains, such proportion is roughly below 3 at any wavelength within PAR range (Figure 2.4), which in 
turn indicates that the difference between both coefficients is rather a couple of percentage points. Such minor discrepancy is indeed in the same order of magnitude as typical experimental measurement errors of optical measurements. Hence, the measured value of $\mathrm{K}_{d}$ can therefore be considered as a reasonable estimate of the value of $K_{0}$. Further, the typical optical path-lengths inside PBR cultures are just few centimetres. Taking these two considerations altogether, one can state that the attenuation coefficient for downward irradiance $\mathrm{K}_{d}$ may properly estimate the absolute amount of radiation available for photosynthesis, the scalar irradiance $\mathrm{E}_{0}$, at a given depth in the biological suspension. This is a critical assumption as we are assessing light availability within photosynthetic cultures by estimating the $\mathrm{K}_{d}$ coefficient and not the scalar one $\mathrm{K}_{0}$. In other words, one can assume that under the studied conditions, most light is principally absorbed by the cells or partially scattered in the forward direction, without achieving a purely diffuse light environment. Thus, downward attenuation coefficient is a reasonable parameter for predicting light distribution within photosynthetic microorganism cultures.

\subsection{Article "Estimation of the light field inside photosynthetic microorganism cultures through Mittag-Leffler functions at depleted light conditions"}

The main results of this research were the modelling of light decay through a Mittag-Leffler function within WT and Olive strain cultures exposed to white LED light at five different intensities. In Figure 3.1, the light field as PAR irradiance value vs. the distance to the illumination source in wild-type cultures is plotted. It can be seen that in WT suspensions for optical densities equal or greater than 1.0, the light field starts to deviate from the pure exponential case, whereas in Olive this happens for optical densities of at least 2.5 (Figure 3.2). The lowest measured light intensity from the original source within the WT cultures 
was $1.24 \mu \mathrm{mol}$ photons $\cdot \mathrm{m}^{-2} \cdot \mathrm{s}^{-1}$ at $3.3 \mathrm{~mm}$, whereas for Olive cultures it was 1.60 at $4.4 \mathrm{~mm}$ (both at the most concentrated cell-density). For these points, light attenuation is far from Lambert-Beer law prediction (a line in the semi-logarithmic plot). Indeed, the obtained values for exponential decay are just $8.1 \%\left(0.1 \mu \mathrm{mol}\right.$ photons $\left.\cdot \mathrm{m}^{-2} \cdot \mathrm{s}^{-1}\right)$ and $19 \%$ $\left(0.3 \mu \mathrm{mol}\right.$ photons $\left.\cdot \mathrm{m}^{-2} \cdot \mathrm{s}^{-1}\right)$ of the respectively experimental values in each strain, which leads to the conclusion that in culture regions where practically all the light has been captured by the cells, the Lambert-Beer law does not satisfactorily represent the remaining light field, at least in terms of PAR range integrated values (dashed lines in Figures 3.1 and 3.1). In fact, this happens in both strains below the euphotic zone, which in our case roughly corresponds to remaining irradiance data lower than $20 \mu \mathrm{mol}$ photons $\cdot \mathrm{m}^{-2} \cdot \mathrm{s}^{-1}$. Alternatively, Mittag-Leffler model (solid lines in the last mentioned plots) is able to correctly predict the remaining downward radiance in both strains at any cell density by fitting the ML $\alpha$ parameter to 0.995 . It is also remarkable that for the case of cultures exposed to a lamp spectrum with a very narrow emission band, the attenuation is purely exponential in the measured conditions (data not shown). This is due to the fact that monochromatic light is taken up by cells at a constant rate given by the attenuation coefficient value at the peak wavelength of the LED emission spectrum (Gaussian-shaped), whereas in the case of white LED light exposure, attenuation varies gradually: cells mainly absorb light at wavelengths close to the peaks corresponding to the pigments present in them. This selective absorption leads to irradiance diminution of the primarily absorbed wavelengths while the less absorbed ones contribute to the remaining light field at deeper distances. In the case of Synechocystis cells, likewise many cyanobacteria which do not possess any photo-active pigment capable of capturing green radiation, the green components of the spectrum are less absorbed, thus light gradually changes from white at the surface to a greener colour at the furthest depth. This leads to a progressively lower attenuation, as cells can hardly capture green light, which explains why a purely exponential decay is not valid in such selectively absorbing media. 


\subsection{Article "Experimental characterisation of Synechocystis sp. PCC 6803 cultures productivity up on light conditions"}

This contribution's objective is to carry out a proper characterisation of Synechocystis cultures of some basic properties of this strain upon different average light intensities. To achieve this goal, it was critical to properly estimate light attenuation along the optical path-length. First of all, this implied a rigorous calibration of the emitted radiation by the light source along the whole PBR glass facing the lamp. Second, to in silico estimate the optical field inside the suspension to work on with mean irradiance values rather than incident ones from the lamp. As previously mentioned, all essays where performed under red light supplemented with a minor amount of blue photons to avoid sub-optimal cell behaviour due to absence of some photosynthesis regulatory procedures.

The first properties to be assessed were the specific growth rate and the related carbon uptake by the cells at different average intensities of continuous red light. Though both variables follow a similar trend as displayed in Figure 4.1, it can be seen that the doubling time -i. e. the growth rate- reaches a maximum value at moderate intensities, whereas carbon uptake keeps rising for even higher irradiance levels. This implies that part of the carbon that has been fixed by the cell is used at high intensities to maintain metabolism and particularly to repair the photosynthesis apparatus. For an average light intensity of roughly $300 \mu \mathrm{mol}$ photons $\cdot \mathrm{m}^{-2} \cdot \mathrm{s}^{-1}$, the doubling time is $6.6 \mathrm{~h}^{-1}$, while maximum carbon uptake reaches $180 \mu \mathrm{mol} \mathrm{CO}_{2} \cdot \mathrm{mg} \mathrm{chl} \mathrm{a}^{-1} \cdot \mathrm{h}^{-1}$ under the most intense radiation. Similarly, growth rates were analysed under intermittent red light, with different HL-LL cycles as depicted in Figure 4.6. The first case of study was to set up a switch cycle of $16 \mathrm{~s}$ at different asymmetric light intensity periods. As such time is a very long period in comparison with photosynthesis functional times, the highest productivity was obtained under symmetric cycles of 110/330 
$\mu$ mol photons $\cdot \mathrm{m}^{-2} \cdot \mathrm{s}^{-1}$, so that cells experienced enough light supply during the whole cycle (upward-pointing triangles). The corresponding doubling time was $4.8 \mathrm{~h}^{-1}$, a very fast growth rate in comparison with typical values reported in literature for this strain. For the case of pure darkness-light varying set-up, lowest biomass formation was measured (downward-pointing triangles).

Another experiment consisted in keeping a HL-LL intensity period of $40 / 440 \mu \mathrm{mol}$ photons $\cdot \mathrm{m}^{-2} \cdot \mathrm{s}^{-1}$ and carrying out trials for several cultures, each experiencing a different switch duration, namely 1, 2, 4, 8 and the already described $16 \mathrm{~s}$ essay (circle markers for this row of essays). Culture exposed to more dynamical cycles grow faster as the quickest alternating periods are closer to the photosynthesis characteristic timing. The latter experiment assessing intermittent radiation was to study how continuous red light affected different cell densities suspensions (star markers). For the studied radiation level, the highest growth rate was achieved at the most diluted culture, implying that more light was averagely available for the cells under the analysed celldensities.

The next property to be studied was pigment concentration within the cells. In this regard, it has to be noted that, as expected, chlorophyll acontent drops as light increases in a linear manner, from more than 2 to 1.4 $\mathrm{mg} \mathrm{chla} \cdot \mathrm{L}^{-1}$ at very high intensities (Figure 4.2). Alternatively, carotenoid concentration follows a proportional trend with respect to irradiance due to their photo-protective role, reaching values of around $0.7 \mathrm{mg} \cdot \mathrm{L}^{-1}$ at higher intensities. Delving deeper into the question of chlorophyll content, this pigment concentration per unit of volume was transformed into mass amount per cell, as depicted in Figure 4.5. This plot displays a strong drop of chlorophyll aupon increasing irradiance values, whereas such amount halves from the maximum values of hundred units to $50 \mathrm{fg} \mathrm{chl} \mathrm{a} \cdot$ cell $^{-1}$ at the highest measure intensity. From a mass balance equation system for chlorophyll, a theoretical minimum amount was calculated taking into account different PSI and PSII proportions at very low and very strong intensities. This value accounts for around $40 \mathrm{fg} \mathrm{chla} \cdot$ cell $^{-1}$ and is in agreement with the fitted decay 
shown in the last Figure 4.5. Right after the basic culture features were estimated, it was decided to study in a systematic way the productivity of the cell in terms of oxygen evolution. To do so, cultures acclimatised for few days to a given intensity were exposed to different exposure intensities by measuring the corresponding P-I curves, as depicted in Figure 4.3. It has to be noted that for each acclimation irradiance value a different colour was used as indicated in the corresponding plot. Plasticity of the photosynthesis can be appreciated as for the most intense exposure radiation, cells grown under the largest intensity trebles the performance for the ones acclimatised to very low irradiance, which is roughly $100 \mu \mathrm{mol} \mathrm{O} 2 \cdot \mathrm{mg} \mathrm{chl} \mathrm{a}{ }^{-1} \cdot \mathrm{h}^{-1}$. Similarly, respiration for the same cultures was also measured, plotted in Figure 4.4. As expected, the different acclimatised cultures show a linearly increasing respiration as the exposure radiation goes up. Moreover, the slope seems to somewhat increase (in absolute terms) as the acclimation intensity does, due to probable higher metabolic maintenance and repair costs to keep a sustained cyclic flow. For the sake of example, respiration levels range from few to $15 \mu \mathrm{mol} \mathrm{O} 2 \cdot \mathrm{mg} \mathrm{chl} \mathrm{a}{ }^{-1} \cdot \mathrm{h}^{-1}$ for the lowest intensity acclimatised cultures, whereas for the cultures kept under the strongest radiation, respiration rises from 35 to $50 \mu \mathrm{mol} \mathrm{O}_{2} \cdot \mathrm{mg} \mathrm{chl} \mathrm{a}^{-1} \cdot \mathrm{h}^{-1}$.

Regarding in silico assessment of $\mathrm{O}_{2}$ evolution, we applied a mechanistic model on oxygen and respiratory process that takes into consideration momentarily and acclimation intensities. It outputs gross and net photosynthetic rates, but dark- and photo-respiratory fluxes, as well. We fitted several parameters to match experimental data of observed $\mathrm{O}_{2}$ evolution as shown in Figure 4.7. Though the fit is not perfect, the simulation follows the overall data trend and additionally, the other modelled process are in agreement with typical values in this organism: while gross yield surpass $320 \mu \mathrm{mol} \mathrm{O} 2 \cdot \mathrm{mg} \mathrm{chl} \mathrm{a}^{-1} \cdot \mathrm{h}^{-1}$, remarkable photorespiration appears after irradiance values above 200 $\mu \mathrm{mol}$ photons $\cdot \mathrm{m}^{-2} \cdot \mathrm{s}^{-1}$ and is thrice the dark-respiratory processes under saturating intensities. These findings appear to be well supported by prior literature. 
Another way to estimate the overall productivity of the culture is to compute the $\mathrm{O}_{2}$ performance in comparison with the energy captured by the cellular pigments at any light intensity. In this regard, assuming an ideal stoichiometric relation of 8 photons to $1 \mathrm{O}_{2}$ molecule, one can plot the relationship between the light absorbed and the $\mathrm{O}_{2}$ productivity. In Figure 4.8, it can be appreciated that as light increases, photosynthesis performance saturates, going from values around $20 \%$ to less than $10 \%$ under very strong radiation in the case of net $\mathrm{O}_{2}$ production capacity. If one takes into account the respiratory mechanisms, that maximal efficiency rises up to around 35\%. Finally, the volumetric yield of the culture can also be in silico estimated for different celldensity suspensions at several incident intensities, as depicted in Figure 4.9. This plot shows that as light goes up, maximum yields are reached at denser suspensions and productivity can approach values close to $700 \mu \mathrm{mol} \mathrm{O} 2 \cdot \mathrm{L}^{-1} \cdot \mathrm{h}^{-1}$ around $15,000 \mathrm{mg} \cdot \mathrm{m}^{-3}$. Interestingly, above the cell concentration at which the maximum performance appears, the overall capacity starts to drop as light is scarcely available, thus a larger amount of cells are below the compensation point, i. e. more cells start to penalize culture yield due to net respiration. Further, the model predicts a constant decay related with the corresponding average respiration of the whole culture.

\subsection{Article "Individual pigment contribution to overall in vivo absorption in Synechocystis sp. PCC 6803 cells"}

In this work, we obtained in a systematic way the absorption percentage of each pigment under physiological conditions. This has important implications as knowing this information opens new possibilities with respect to modelling photosynthesis processes that are intimately linked with light spectra. Package effect was first computed following Morel's approach, as outlined in the corresponding section, and the obtained result is in agreement with other microorganisms possessing a 
similar chlorophyll internal concentration. In Figure 5.2, it can be observed that the package effect factor for the red and green range is close to 0.9 , yet for blue light this correction factor is below 0.8 , indicating that there is a considerable difference between in vitro and in vivo absorption for chlorophyll aand carotenoids under blue radiation. Knowing the package effect factor for the whole PAR range, one can estimate the in vivo absorption signature for each pigment as depicted in Figure 5.3. This plot shows the individual contribution to cell absorption and delivers rather precise results as the reconstructed spectrum overlaps practically for the whole PAR range the in vivo curve. The only exception was the blue-green waveband, primarily absorbed by carotenoids. Further, it is possible to estimate the percentage of the radiation harvested by phycobilisomes as shown in Figure 5.4. This is another remarkable feature since PBS can deliver excitation energy in a more or less balanced manner towards both photosystems, while chlorophyll aexcitation can be hardly transferred between photosystem I and II, principally through spill-over energy mechanisms, which have limited range and only occur during some type of state-transitions processes. In summary, yellow to red light seems to be optimal for photosynthesis performance. However, it is known that yellow light does not promote an electron flow as high as the one arising under orange light. This could be in principle attributable to a minor but necessary excitation of chlorophyll amolecules in PSI, which does not occur under yellow photons. Yet, further investigation is required to shed light on this matter and by doing so assess the optimal wavelength combination for photosynthesis in cyanobacterial cells. Finally, we calculated the average absorption cross-section of Synechocystis cells exposed to all the different light sources shown in Figure 5.1. There it can be seen that under radiation emitted by traditional white spectrum lamps, the cellular cross-section is roughly $0.01 \mathrm{~m}^{2} \cdot \mathrm{mg} \mathrm{chl} \mathrm{a}^{-1}$. In this sense, halogen $(\mathrm{H})$ and warm white LED lamps support a higher amount of light harvesting by phycobilisomes, fact which is in principle desired for higher photosynthetic yields. Alternatively, cells under monochromatic LED radiation own disparate values for the absorption cross-section, as the value for the ones exposed to LEDs emit- 
ting blue or orange irradiance almost doubles the computed figure for cells facing broader white lamps and for cultures experiencing daylight. However, poorly absorbed wavebands such as cyan and green light lead to very low absorption. Besides, orange radiation is principally absorbed by phycobilins, while blue irradiance is harvested by chlorophyll molecules, mostly PSI complexes. In this regard, the worst monochromatic light source for photosynthesis performance would be blue-green, i. e. turquoise (T), radiation as it is principally captured by carotenoid chromophores, which display a very low transfer efficiency to chlorophyll amolecules. It is noteworthy to mention that exposing cells to different light sources may affect final pigment composition, which would lead to different in vivo absorption spectra and so the cross-section would vary. But due to lack of data and expecting only minor dissimilarities, the resulting average absorption cross-sections should be similar to the ones displayed in Figure 5.1. In summary, predicting the radiation harvested by each of the main chromophores located within photosynthetic microorganisms would shed light on the fate of the absorbed energy and hence, aid in the understanding on where such excitation is finally ending up within the whole photosynthesis machinery. 



\section{7}

\section{Conclusions}

In the present doctoral thesis, we have studied optical phenomena from different perspectives: the light field distribution along the culture, the effect of light on basic cell characteristics and also the participation of each photo-active pigment in the cell absorption within Synechocystis cells.

In the first article, "Light distribution and spectral composition within cultures of micro-algae: Quantitative modelling of the light field in photobioreactors", we showed that by means of a rather simple modelling framework, we were able to estimate within a reasonable accuracy the light field inside two different strains of Synechocystis, namely wild-type and Olive strain. Moreover, the prediction was valid for all cell-densities and studied light sources. However, the prediction failed to precisely calculate the remaining irradiance at very depleted radiation conditions. To this purpose, the exponential approach was substituted with a Mittag-Leffler function. This methodology was later described in a second contribution, entitled "Estimation of the light field inside photosynthetic microorganism cultures through Mittag-Leffler functions at depleted light conditions", where the mathematical formalism using fractional calculus was explained. The generalisation of the Lambert-Beer law delivered a very accurate description of the field in both strains for all cell-densities with a unique fitting parameter. 
In the third article, "Experimental characterisation of Synechocystis sp. PCC 6803 cultures productivity up on light conditions", we assessed the basic properties of Synechocystis cultures up on different irradiance values and estimated in silico the optimal values for $\mathrm{O}_{2}$ productivity at different scales, i. e. the maximum performance for a single cell and for the whole bioreactor. In the latter case, we obtained the overall performance for different cell densities and light intensities.

In the last contribution, we calculated the package effect of the cell to reconstruct the Synechocystis in vivo absorption spectrum from in vitro signatures of the cell pigments and by doing so, calculating the concentration of the main chromophores. Additionally, the contribution of each pigment to overall light absorption depending on the type of light source employed was also assessed.

It is worth mentioning that this thesis contributes to this field by bringing into play different methodologies that combine pure optical aspects with biological ones as an another step towards integrative opticalphotosynthetic modelling. In this regard, the here presented research generalises light treatment to account with its spectral properties.

Future work will have to describe in a more detailed manner photosynthesis machinery and its interaction with light: non-photochemical quenching, state transitions or the circadian clock, among other critical processes, do depend on the quantity and quality of the radiation cells are exposed. In summary, the different procedures cells own to protect themselves against photodamage and optimise excitation energy balance are directly related with light. Due to their relevance in the different electron flow routes and so in photosynthesis efficiency, further investigation is needed to support the design of optimised mutant strains for potential industrial applications of photosynthetic microorganisms. 


\section{Bibliography}

Aasen, A., Jensen, S. L., 1966. Carotenoids of flexibacteria. iv. the carotenoids of two further pigment types. Acta Chemica Scandinavica $20(8), 2322-2324$.

Adams, W. W., Demmig-Adams, B., Logan, B. A., Barker, D. H., Osmond, C. B., 1999. Rapid changes in xanthophyll cycle-dependent energy dissipation and photosystem II efficiency in two vines, Stephania japonica and Smilax australis, growing in the understory of an open eucalyptus forest. Plant, Cell \& Environment 22 (2), 125-136.

Adir, N., 2005. Elucidation of the molecular structures of components of the phycobilisome: reconstructing a giant. Photosynthesis Research 85 (1), 15-32.

Ahmad, Z., Fraser, R. S., 1982. An iterative radiative transfer code for ocean-atmosphere systems. Journal of the Atmospheric Sciences 39 (3), 656-665.

Allahverdiyeva, Y., Ermakova, M., Eisenhut, M., Zhang, P., Richaud, P., Hagemann, M., Cournac, L., Aro, E.-M., 2011. Interplay between flavodiiron proteins and photorespiration in Synechocystis sp. PCC 6803. Journal of Biological Chemistry 286 (27), 24007-24014.

Allen, J. F., Mullineaux, C. W., 2004. Probing the mechanism of state transitions in oxygenic photosynthesis by chlorophyll fluorescence spectroscopy, kinetics and imaging. In: Chlorophyll a Fluorescence. Springer, pp. 447-461. 
Antoine, D., Babin, M., Berthon, J. F., Bricaud, A., Gentili, B., Loisel, H., Maritorena, S., Stramski, D., 2014. Shedding light on the sea: André morel's legacy to optical oceanography. Annual Review of Marine Science 6, 1-21.

Arteni, A. A., Ajlani, G., Boekema, E. J., 2009. Structural organisation of phycobilisomes from Synechocystis sp. strain PCC6803 and their interaction with the membrane. Biochimica et Biophysica Acta 1787 (4), 272-279.

Ashby, M. K., Mullineaux, C. W., 1999. Cyanobacterial ycf27 gene products regulate energy transfer from phycobilisomes to photosystems I and II. FEMS Microbiology Letters 181 (2), 253-260.

Astier, C., Elmorjani, K., Meyer, I., Joset, F., Herdman, M., 1984. Photosynthetic mutants of the cyanobacteria Synechocystis sp. strains PCC 6714 and PCC 6803: sodium p-hydroxymercuribenzoate as a selective agent. Journal of Bacteriology 158 (2), 659-664.

Atangana, A., Baleanu, D., 2016. New fractional derivatives with nonlocal and non-singular kernel: Theory and application to heat transfer model. Thermal Science, 18.

Bald, D., Kruip, J., Rögner, M., 1996. Supramolecular architecture of cyanobacterial thylakoid membranes: how is the phycobilisome connected with the photosystems? Photosynthesis Research 49 (2), 103118.

Baleanu, D., Diethelm, K., Scalas, E., Trujillo, J. J., 2012. Models and numerical methods. World Scientific 3, 10-16.

Beardall, J., Burger-Wiersma, T., Rijkeboer, M., Sukenik, A., Lemoalle, J., Dubinsky, Z., Fontvielle, D., 1994. Studies on enhanced postillumination respiration in microalgae. Journal of Plankton Research 16 (10), 1401-1410.

Bennett, A., Bogorad, L., 1973. Complementary chromatic adaptation in a filamentous blue-green alga. The Journal of Cell Biology 58 (2), 419-435. 
Benschop, J. J., Badger, M. R., Price, G. D., 2003. Characterisation of $\mathrm{CO}_{2}$ and $\mathrm{HCO}^{-}$uptake in the cyanobacterium Synechocystis sp. PCC6803. Photosynthesis Research 77 (2-3), 117-126.

Berberan-Santos, M. N., 2005. Properties of the Mittag-Leffler relaxation function. Journal of Mathematical Chemistry 38 (4), 629-635.

Bernát, G., Waschewski, N., Rögner, M., 2009. Towards efficient hydrogen production: the impact of antenna size and external factors on electron transport dynamics in Synechocystis PCC 6803. Photosynthesis Research 99 (3), 205-216.

Bidigare, R. R., Ondrusek, M. E., Morrow, J. H., Kiefer, D. A., 1990. In vivo absorption properties of algal pigments. Ocean Optics $\mathrm{X}$, Proc. SPIE 1302, 290-302.

Blanken, W., Postma, P. R., de Winter, L., Wijffels, R. H., Janssen, M., 2016. Predicting microalgae growth. Algal Research 14, 28-38.

Boehm, M., Nield, J., Zhang, P., Aro, E.-M., Komenda, J., Nixon, P. J., 2009. Structural and mutational analysis of band 7 proteins in the cyanobacterium Synechocystis sp. strain PCC 6803. Journal of bacteriology 191 (20), 6425-6435.

Borovoi, A., 2002. On the extinction of radiation by a homogeneous but spatially correlated random medium: comment. Journal of the Optical Society of America A 19 (12), 2517-2520.

Bricaud, A., Bédhomme, A.-L., Morel, A., 1988. Optical properties of diverse phytoplanktonic species: experimental results and theoretical interpretation. Journal of Plankton Research 10 (5), 851-873.

Bricaud, A., Claustre, H., Ras, J., Oubelkheir, K., 2004. Natural variability of phytoplanktonic absorption in oceanic waters: Influence of the size structure of algal populations. Journal of Geophysical Research: Oceans 109 (C11), 12 pages, c11010.

Bricaud, A., Morel, A., 1986. Light attenuation and scattering by phytoplanktonic cells: a theoretical modeling. Applied Optics 25 (4), 571580. 
Bricaud, A., Stramski, D., 1990. Spectral absorption coefficients of living phytoplankton and nonalgal biogenous matter: A comparison between the peru upwelling areaand the sargasso sea. Limnology and Oceanography 35 (3), 562-582.

Brody, M., Emerson, R., 1959. The effect of wavelength intensity of light on the proportion of pigments in Porphyridium cruentum. American Journal of Botany 46, 433-440.

Broess, K., Trinkunas, G., van der Weij-de, C. D., Dekker, J. P., van Hoek, A., van Amerongen, H., et al., 2006. Excitation energy transfer and charge separation in photosystem II membranes revisited. Biophysical Journal 91 (10), 3776-3786.

Bryant, D. A., Glazer, A. N., Eiserling, F. A., 1976. Characterization and structural properties of the major biliproteins of Anabaena sp. Archives of Microbiology 110 (1), 61-75.

Bryant, D. A., Guglielmi, G., de Marsac, N. T., Castets, A.-M., CohenBazire, G., 1979. The structure of cyanobacterial phycobilisomes: a model. Archives of Microbiology 123 (2), 113-127.

Buck, D. P., Smith, G. D., 1995. Evidence for a na+/h+ electrogenic antiporter in an alkaliphilic cyanobacterium Synechocystis. FEMS Microbiology Letters 128 (3), 315-320.

Calık, A. E., Sirin, H., Ertik, H., Sen, M., 2016. Analysis of charge variation in fractional order LC electrical circuit. Revista Mexicana de Física 62 (5), 437-441.

Capuano, V., Braux, A.-S., de Marsac, N. T., Houmard, J., 1991. The "anchor polypeptide" of cyanobacterial phycobilisomes. molecular characterization of the Synechococcus sp. PCC 6301 apce gene. Journal of Biological Chemistry 266 (11), 7239-7247.

Casasanta, G., Ciani, D., Garra, R., 2012. Non-exponential extinction of radiation by fractional calculus modelling. Journal of Quantitative Spectroscopy and Radiative Transfer 113 (2), 194-197. 
Cerullo, G., Polli, D., Lanzani, G., De Silvestri, S., Hashimoto, H., Cogdell, R. J., 2002. Photosynthetic light harvesting by carotenoids: detection of an intermediate excited state. Science 298 (5602), 23952398.

Červenỳ, J., Sinetova, M. A., Zavřel, T., Los, D. A., 2015. Mechanisms of high temperature resistance of Synechocystis sp. PCC 6803: an impact of histidine kinase 34. Life 5 (1), 676-699.

Chábera, P., Durchan, M., Shih, P. M., Kerfeld, C. A., Polívka, T., 2011. Excited-state properties of the 16kda red carotenoid protein from Arthrospira maxima. Biochimica et Biophysica Acta (BBA)Bioenergetics 1807 (1), 30-35.

Chisti, Y., 2007. Biodiesel from microalgae. Biotechnology Advances 25 (3), 294-306.

Collins, A. M., Liberton, M., Jones, H. D., Garcia, O. F., Pakrasi, H. B., Timlin, J. A., 2012. Photosynthetic pigment localization and thylakoid membrane morphology are altered in Synechocystis 6803 phycobilisome mutants. Plant Physiology 158 (4), 1600-1609.

Cornet, J., Dussap, C., Dubertret, G., 1992. A structured model for simulation of cultures of the cyanobacterium Spirulina platensis in photobioreactors: I. coupling between light transfer and growth kinetics. Biotechnology and Bioengineering 40 (7), 817-825.

Dauchet, J., Blanco, S., Cornet, J.-F., El Hafi, M., Eymet, V., Fournier, R., 2013. The practice of recent radiative transfer Monte Carlo advances and its contribution to the field of microorganisms cultivation in photobioreactors. Journal of Quantitative Spectroscopy and Radiative Transfer 128, 52-59.

David, L., Marx, A., Adir, N., 2011. High-resolution crystal structures of trimeric and rod phycocyanin. Journal of molecular biology 405 (1), 201-213. 
Davies-Colley, R., Pridmore, R., Hewitt, J., 1986. Optical properties of some freshwater phytoplanktonic algae. Hydrobiologia 133 (2), 165178.

de Mooij, T., de Vries, G., Latsos, C., Wijffels, R. H., Janssen, M., 2016. Impact of light color on photobioreactor productivity. Algal Research 15, 32-42.

Dexter, J., Fu, P., 2009. Metabolic engineering of cyanobacteria for ethanol production. Energy \& Environmental Science 2 (8), 857-864.

Dubinsky, Z., Stambler, N., 2009. Photoacclimation processes in phytoplankton: mechanisms, consequences, and applications. Aquatic Microbial Ecology 56 (2-3), 163-176.

Eilers, P., Peeters, J., 1988. A model for the relationship between light intensity and the rate of photosynthesis in phytoplankton. Ecological Modelling 42 (3-4), 199-215.

Elmorjani, K., Thomas, J.-C., Sebban, P., 1986. Phycobilisomes of wild type and pigment mutants of the cyanobacterium Synechocystis PCC 6803. Archives of Microbiology 146 (2), 186-191.

Ermakova, M., Huokko, T., Richaud, P., Bersanini, L., Howe, C. J., Lea-Smith, D. J., Peltier, G., Allahverdiyeva, Y., 2016. Distinguishing the roles of thylakoid respiratory terminal oxidases in the cyanobacterium Synechocystis sp. PCC 6803. Plant Physiology 171 (2), 13071319.

Faccio, G., Kämpf, M. M., Piatti, C., Thöny-Meyer, L., Richter, M., 2014. Tyrosinase-catalyzed site-specific immobilization of engineered c-phycocyanin to surface. Scientific Reports 4, 5370, 8 pages.

Falkowski, P. G., Dubinsky, Z., Santostefano, G., 1985. Light-enhanced dark respiration in phytoplankton. Internationale Vereinigung für theoretische und angewandte Limnologie: Verhandlungen 22 (5), 2830-2833. 
Ferreira, K. N., Iverson, T. M., Maghlaoui, K., Barber, J., Iwata, S., 2004. Architecture of the photosynthetic oxygen-evolving center. Science 303 (5665), 1831-1838.

Ficek, D., Kaczmarek, S., Ston-Egiert, J., Wozniak, B., Majchrowski, R., Dera, J., 2004. Spectra of light absorption by phytoplankton pigments in the baltic; conclusions to be drawn from a gaussian analysis of empirical data. Oceanologia 46 (4), 533-555.

Fisher, T., Shurtz-Swirski, R., Gepstein, S., Dubinsky, Z., 1989. Changes in the levels of ribulose-1, 5-bisphosphate carboxylase/oxygenase (Rubisco) in Tetraedron minimum (Chlorophyta) during light and shade adaptation. Plant and Cell Physiology 30 (2), 221-228.

Fork, D., 1963. Observations of the function of chlorophyll a and accessory pigments in photosynthesis. In: Photosynthetic Mechanisms of Green Plants. National Academy of Science-National Research Council. Vol. 1145. pp. 352-361.

Fuente, D., Keller, J., Conejero, J. A., Rögner, M., Rexroth, S., Urchueguía, J. F., 2017. Light distribution and spectral composition within cultures of micro-algae: Quantitative modelling of the light field in photobioreactors. Algal Research 23, 166-177.

Fuente, D., Lizama, C., Urchueguía, J. F., Conejero, J. A., 2018. Estimation of the light field inside photosynthetic microorganism cultures through Mittag-Leffler functions at depleted light conditions. Journal of Quantitative Spectroscopy and Radiative Transfer 204 (Supplement C), 23-26.

Fujita, Y., Murakami, A., 1987. Regulation of electron transport composition in cyanobacterial photosynthetic system: stoichiometry among photosystem I and II complexes and their light-harvesting antennae and cytochrome b6/f complex. Plant and Cell Physiology 28 (8), 1547-1553.

García-Camacho, F., Sánchez-Mirón, A., Molina-Grima, E., CamachoRubio, F., Merchuck, J., 2012. A mechanistic model of photosynthesis 
in microalgae including photoacclimation dynamics. Journal of Theoretical Biology 304, 1-15.

Genty, B., Briantais, J.-M., Baker, N. R., 1989. The relationship between the quantum yield of photosynthetic electron transport and quenching of chlorophyll fluorescence. Biochimica et Biophysica Acta (BBA)-General Subjects 990 (1), 87-92.

Gitelson, A., Hu, Q., Richmond, A., 1996. Photic volume in photobioreactors supporting ultrahigh population densities of the photoautotroph Spirulina platensis. Applied and Environmental Microbiology 62, 1570-1573.

Glöckle, W. G., Nonnenmacher, T. F., 1995. A fractional calculus approach to self-similar protein dynamics. Biophysical Journal 68 (1), 46-53.

Gobets, B., van Grondelle, R., 2001. Energy transfer and trapping in photosystem i. Biochimica et Biophysica Acta (BBA)-Bioenergetics 1507 (1), 80-99.

Gobets, B., van Stokkum, I. H., van Mourik, F., Dekker, J. P., van Grondelle, R., 2003. Excitation wavelength dependence of the fluorescence kinetics in photosystem I particles from Synechocystis PCC 6803 and Synechococcus elongatus. Biophysical Journal 85 (6), 3883-3898.

Golden, S. S., 1995. Light-responsive gene expression in cyanobacteria. Journal of Bacteriology 177 (7), 1651.

Grigorieva, G., Shestakov, S., 1982. Transformation in the cyanobacterium Synechocystis sp. 6803. FEMS Microbiology Letters 13 (4), 367370.

Grobbelaar, J. U., Nedbal, L., Tichỳ, V., 1996. Influence of high frequency light/dark fluctuations on photosynthetic characteristics of microalgae photoacclimated to different light intensities and implications for mass algal cultivation. Journal of Applied Phycology 8 (4-5), 335-343. 
Harmut, A., Lichtenthaler, K., 1987. Chlorophylls and carotenoids: pigments of photosynthetic membranes. Method Enzymol 148, 350-383.

Haubold, H. J., Mathai, A. M., Saxena, R. K., 2011. Mittag-Leffler functions and their applications. Journal of Applied Mathematics ID 298628, 51 pages.

Heinrich, J. M., Niizawa, I., Botta, F. A., Trombert, A. R., Irazoqui, H. A., 2012. Analysis and design of photobioreactors for microalgae production II: experimental validation of a radiation field simulator based on a Monte Carlo algorithm. Photochemistry and Photobiology 88 (4), 952-960.

Helman, Y., Tchernov, D., Reinhold, L., Shibata, M., Ogawa, T., Schwarz, R., Ohad, I., Kaplan, A., 2003. Genes encoding a-type flavoproteins are essential for photoreduction of $\mathrm{O}_{2}$ in cyanobacteria. Current Biology 13 (3), 230-235.

Hertzberg, S., Liaaen-Jensen, S., Siegelman, H., 1971. The carotenoids of blue-green algae. Phytochemistry 10 (12), 3121-3127.

Hihara, Y., Sonoike, K., Ikeuchi, M., 1998. A novel gene, pmga, specifically regulates photosystem stoichiometry in the cyanobacterium Synechocystis species PCC 6803 in response to high light. Plant Physiology 117 (4), 1205-1216.

Hilfer, R., 2000. Fractional diffusion based on Riemann-Liouville fractional derivatives. Photochemistry and Photobiology 104 (16), 39143917.

Hiyama, T., Nishimura, M., Chance, B., 1969. Determination of carotenes by thin-layer chromatography. Analytical Biochemistry 29 (2), 339-342.

Hoepffner, N., Sathyendranath, S., 1991. Effect of pigment composition on absorption properties of phytoplankton. Marine Ecology Progress Series, 11-23.

Ibrahim, R., Ozel, C., 2016. On multi-order fractional differential operators in the unit disk. Filomat 30 (1), 73-81. 
Ikeuchi, M., Tabata, S., 2001. Synechocystis sp. PCC 6803-a useful tool in the study of the genetics of cyanobacteria. Photosynthesis Research 70 (1), 73-83.

Iluz, D., Alexandrovich, I., Dubinsky, Z., 2012. The enhancement of photosynthesis by fluctuating light. In: Artificial Photosynthesis. InTech, pp. 110-134.

Jackson, S. A., Hervey, J. R., Dale, A. J., Eaton-Rye, J. J., 2014. Removal of both Ycf48 and Psb27 in Synechocystis sp. PCC 6803 disrupts photosystem II assembly and alters $\mathrm{q}_{a}^{-}$oxidation in the mature complex. FEBS Letters 588 (20), 3751-3760.

Jiang, H.-B., Song, W.-Y., Cheng, H.-M., Qiu, B.-S., 2015. The hypothetical protein Ycf46 is involved in regulation of $\mathrm{CO}_{2}$ utilization in the cyanobacterium Synechocystis sp. PCC 6803. Planta 241 (1), 145-155.

Jordan, P., Fromme, P., Witt, H. T., Klukas, O., Saenger, W., Krauß, N., 2001. Three-dimensional structure of cyanobacterial photosystem I at 2.5 å resolution. Nature 411 (6840), 909-917.

Jorgensen, B. B., Cohen, Y., Des Marais, D. J., 1987. Photosynthetic action spectra and adaptation to spectral light distribution in a benthic cyanobacterial mat. Applied and Environmental Microbiology 53, 879-886.

Kakitani, T., Honig, B., Crofts, A. R., 1982. Theoretical studies of the electrochromic response of carotenoids in photosynthetic membranes. Biophysical Journal 39 (1), 57-63.

Kana, T. M., Glibert, P. M., 1987. Effect of irradiances up to $2000 \mu \mathrm{em}^{-2}$. $s^{-1}$ on marine Synechococcus wh7803-I. Growth, pigmentation, and cell composition. Deep Sea Research Part A. Oceanographic Research Papers 34 (4), 479-495.

Kaneko, T., Sato, S., Kotani, H., Tanaka, A., Asamizu, E., Nakamura, Y., Miyajima, N., Hirosawa, M., Sugiura, M., Sasamoto, S., et al., 1996. Sequence analysis of the genome of the unicellular cyanobacterium Synechocystis sp. strain PCC6803 II. sequence determination of the 
entire genome and assignment of potential protein-coding regions. DNA Research 3 (3), 109-136.

Kilian, O., Steunou, A.-S., Fazeli, F., Bailey, S., Bhaya, D., Grossman, A. R., 2007. Responses of a thermophilic Synechococcus isolate from the microbial mat of octopus spring to light. Applied and Environmental Microbiology 73 (13), 4268-4278.

Kim, H.-W., Park, S., Rittmann, B. E., 2015. Multi-component kinetics for the growth of the cyanobacterium Synechocystis sp. PCC6803. Environmental Engineering Research 20 (4), 347-355.

Kim, H. W., Vannela, R., Zhou, C., Harto, C., Rittmann, B. E., 2010. Photoautotrophic nutrient utilization and limitation during semicontinuous growth of Synechocystis sp. PCC6803. Biotechnology and Bioengineering 106 (4), 553-563.

Kirk, J. T., 2003. The vertical attenuation of irradiance as a function of the optical properties of the water. Limnology and Oceanography $48(1), 9-17$.

Kirk, J. T. O., 1975a. A theoretical analysis of the contribution of algal cells to the attenuation of light within natural waters I. general treatment of suspensions of pigmented cells. New Phytologist 75 (1), $11-20$.

Kirk, J. T. O., 1975b. A theoretical analysis of the contribution of algal cells to the attenuation of light within natural waters II. spherical cells. New Phytologist 75 (1), 21-36.

Kirk, J. T. O., 1977. Use of a quanta meter to measure attenuation and underwater reflectance of photosynthetically active radiation in some inland and coastal south-eastern Australian waters. Australian Journal of Marine and Freshwater Research 28, 9-21.

Kirk, J. T. O., 1981. A Monte Carlo study of the nature of the underwater light field in, and the relationships between optical properties of, turbid yellow waters. Australian Journal of Marine and Freshwater Research 32, 517--532. 
Kirk, J. T. O., 1984. Attenuation of solar radiation in scatteringabsorbing waters: a simplified procedure for its calculation. Applied Optics 23, 3737-3739.

Kirk, J. T. O., 1994. Light and photosynthesis in aquatic ecosystems. Cambridge UK, Cambridge University Press.

Kirst, H., Formighieri, C., Melis, A., 2014. Maximizing photosynthetic efficiency and culture productivity in cyanobacteria upon minimizing the phycobilisome light-harvesting antenna size. Biochimica et Biophysica Acta (BBA)-Bioenergetics 1837, 1653-1664.

Kłodawska, K., Kovács, L., Várkonyi, Z., Kis, M., Sozer, Ö., LaczkóDobos, H., Kóbori, O., Domonkos, I., Strzałka, K., Gombos, Z., et al., 2014. Elevated growth temperature can enhance photosystem I trimer formation and affects xanthophyll biosynthesis in cyanobacterium Synechocystis sp. PCC6803 cells. Plant and Cell Physiology 56 (3), 558-571.

Knoechel, R., Quinn, E., 1989. Carbon dynamics of logarithmetic and stationary phase phytoplankton as determined by track autoradiography. Cytometry 10 (5), 612-621.

Kok, B., 1953. Experiments on photosynthesis by Chlorella in flashing light. Algal culture: from laboratory to pilot plant 600, 63-75.

Kondo, K., Ochiai, Y., Katayama, M., Ikeuchi, M., 2007. The membraneassociated cpcg2-phycobilisome in Synechocystis: a new photosystem I antenna. Plant Physiology 144 (2), 1200-1210.

Kopečná, J., Komenda, J., Bučinská, L., Sobotka, R., 2012. Long-term acclimation of the cyanobacterium Synechocystis sp. PCC 6803 to high light is accompanied by an enhanced production of chlorophyll that is preferentially channeled to trimeric photosystem I. Plant Physiology 160 (4), 2239-2250.

Kostinski, A. B., 2001. On the extinction of radiation by a homogeneous but spatially correlated random medium. Journal of the Optical Society of America A 18 (8), 1929-1933. 
Kostinski, A. B., 2002. On the extinction of radiation by a homogeneous but spatially correlated random medium: reply to comment. Journal of the Optical Society of America A 19 (12), 2521-2525.

Kubín, Š., Borns, E., Doucha, J., Seiss, U., 1983. Light absorption and production rate of Chlorella vulgaris in light of different spectral composition. Biochemie und Physiologie der Pflanzen 178 (2-3), 193-205.

Kwon, J.-H., Bernát, G., Wagner, H., Rögner, M., Rexroth, S., 2013. Reduced light-harvesting antenna: Consequences on cyanobacterial metabolism and photosynthetic productivity. Algal Research 2, 188195.

Kwon, J.-H., Rögner, M., Rexroth, S., 2012. Direct approach for bioprocess optimization in a continuous flat-bed photobioreactor system. Journal of Biotechnology 162 (1), 156-162.

Laisk, A., Nedbal, L., Govindjee, 2009. Photosynthesis in silico: Understanding Complexity from Molecules to Ecosystem. Springer.

Lang, K. R., 1999. Astrophysical formulae-volume i: Radiation, gas processes and high energy astrophysics. Astronomy and Astrophysics Library.

Lauceri, R., Bresciani, M., Lami, A., Morabito, G., 2017. Chlorophyll a interference in phycocyanin and allophycocyanin spectrophotometric quantification. Journal of Limnology.

Lea-Smith, D. J., Bombelli, P., Dennis, J. S., Scott, S. A., Smith, A. G., Howe, C. J., 2014. Phycobilisome-deficient strains of Synechocystis sp. PCC 6803 have reduced size and require carbon-limiting conditions to exhibit enhanced productivity. Plant Physiology 165, 705-714.

Liberton, M., Howard Berg, R., Heuser, J., Roth, R., Pakrasi, H. B., 2006. Ultrastructure of the membrane systems in the unicellular cyanobacterium Synechocystis sp. strain PCC 6803. Protoplasma 227 (2), 129 138.

Liptay, W., 1969. Elektrochromie - solvatochromie. Angewandte Chemie 81 (6), 195-206. 
Lohrenz, S. E., Weidemann, A. D., Tuel, M., 2003. Phytoplankton spectral absorption as influenced by community size structure and pigment composition. Journal of Plankton Research 25 (1), 35-61.

Lopo, M., Montagud, A., Navarro, E., Cunha, I., Zille, A., De Córdoba, P. F., Moradas-Ferreira, P., Tamagnini, P., Urchueguía, J. F., 2012. Experimental and modeling analysis of Synechocystis sp. PCC 6803 growth. Journal of Molecular Microbiology and Biotechnology $22(2), 71-82$.

Ma, W., Ogawa, T., Shen, Y., Mi, H., 2007. Changes in cyclic and respiratory electron transport by the movement of phycobilisomes in the cyanobacterium Synechocystis sp. strain PCC 6803. Biochimica et Biophysica Acta 1767 (6), 742-749.

MacColl, R., 1983. Stability of allophycocyanin's quaternary structure. Archives of Biochemistry and Biophysics 223 (1), 24-32.

MacColl, R., 1998. Cyanobacterial phycobilisomes. Journal of Structural Biology 124 (2), 311-334.

MacColl, R., 2004. Allophycocyanin and energy transfer. Biochimica et Biophysica Acta (BBA)-Bioenergetics 1657 (2), 73-81.

Mainardi, F., Gorenflo, R., Vivoli, A., 2005. Renewal processes of Mittag-Leffler and Wright type. Fractional Calculus and Applied Analysis 8 (1), 7-38.

Markou, G., 2014. Effect of various colors of light-emitting diodes (LEDs) on the biomass composition of Arthrospira platensis cultivated in semi-continuous mode. Applied Biochemistry and Biotechnology $172,2758-2768$.

Martínez, L., Morán, A., García, A. I., 2012. Effect of light on Synechocystis sp. and modelling of its growth rate as a response to average irradiance. Journal of Applied Phycology 24 (1), 125-134.

Mauzerall, D., Greenbaum, N. L., 1989. The absolute size of a photosynthetic unit. Biochimica et Biophysica Acta (BBA)-Bioenergetics 974 (2), 119-140. 
McConnell, M. D., Koop, R., Vasil'ev, S., Bruce, D., 2002. Regulation of the distribution of chlorophyll and phycobilin-absorbed excitation energy in cyanobacteria. a structure-based model for the light state transition. Plant physiology 130 (3), 1201-1212.

McCree, K. J., 1971. The action spectrum, absorptance and quantum yield of photosynthesis in crop plants. Agricultural Meteorology 9, 191-216.

Metzler, R., Klafter, J., 2000. The random walk's guide to anomalous diffusion: a fractional dynamics approach. Physics Reports 339 (1), $1-77$.

Mishchenko, M. I., 2014a. Directional radiometry and radiative transfer: The convoluted path from centuries-old phenomenology to physical optics. Journal of Quantitative Spectroscopy and Radiative Transfer 146, 4 - 33, electromagnetic and Light Scattering by Nonspherical Particles XIV.

Mishchenko, M. I., 2014b. Electromagnetic scattering by particles and particle groups: an introduction. Cambridge University Press.

Mishra, S., Mishra, D. R., Lee, Z., Tucker, C. S., 2013. Quantifying cyanobacterial phycocyanin concentration in turbid productive waters: A quasi-analytical approach. Remote Sensing of Environment 133, 141-151.

Mitchell, B. G., Kiefer, D. A., 1988. Variability in pigment specific particulate fluorescence and absorption-spectra in the northeastern pacificocean. Deep-Sea Research Part a-Oceanographic Research Papers 35 (5), 665-689.

Miyake, C., Horiguchi, S., Makino, A., Shinzaki, Y., Yamamoto, H., Tomizawa, K.-i., 2005. Effects of light intensity on cyclic electron flow around PSI and its relationship to non-photochemical quenching of Chl fluorescence in tobacco leaves. Plant and Cell Physiology 46 (11), 1819-1830. 
Moal, G., Lagoutte, B., 2012. Photo-induced electron transfer from photosystem I to NADP+: Characterization and tentative simulation of the in vivo environment. Biochimica et Biophysica Acta (BBA)Bioenergetics 1817 (9), 1635-1645.

Mohamed, H. E., van de Meene, A. M., Roberson, R. W., Vermaas, W. F., 2005. Myxoxanthophyll is required for normal cell wall structure and thylakoid organization in the cyanobacterium Synechocystis sp. strain PCC 6803. Journal of Bacteriology 187 (20), 6883-6892.

Molina Grima, E., Acién Fernández, F. G., García Camacho, F., Chisti, Y., 1999. Photobioreactors: light regime, mass transfer, and scaleup. Journal of Biotechnology 70, 231-247.

Molina Grima, E., García Camacho, F., Sánchez Pérez, J. A., Fernández Sevilla, J. M., Acién Fernández, F. G., Contreras Gómez, A., 1994. A mathematical model of microalgal growth in light-limited chemostat culture. Journal of Chemical Technology and Biotechnology 61, 167-173.

Montagud, A., Zelezniak, A., Navarro, E., de Córdoba, P. F., Urchueguía, J. F., Patil, K. R., 2011. Flux coupling and transcriptional regulation within the metabolic network of the photosynthetic bacterium Synechocystis sp. pcc6803. Biotechnology Journal 6 (3), 330342.

Morel, A., 1987. Chlorophyll-specific scattering coefficient of phytoplankton. A simplified theoretical approach. Deep Sea Research Part A. Oceanographic Research Papers 34 (7), 1093-1105.

Morel, A., 1991. Light and marine photosynthesis: a spectral model with geochemical and climatological implications. Progress in Oceanography 26, 263-306.

Morel, A., Bricaud, A., 1981a. Theoretical results concerning light absorption in a discrete medium, and application to specific absorption of phytoplankton. Deep Sea Research Part A. Oceanographic Research Papers 28, 1375-1393. 
Morel, A., Bricaud, A., 1981b. Theoretical results concerning the optics of phytoplankton, with special reference to remote sensing applications. In: Oceanography from Space. Springer, pp. 313-327.

Mullineaux, C. W., 1992. Excitation energy transfer from phycobilisomes to photosystem I in a cyanobacterium. Biochimica et Biophysica Acta (BBA)-Bioenergetics 1100 (3), 285-292.

Mullineaux, C. W., 2008. Phycobilisome-reaction centre interaction in cyanobacteria. Photosynthesis Research 95 (2-3), 175-182.

Münzner, P., Voigt, J., 1992. Blue light regulation of cell division in Chlamydomonas reinhardtii. Plant Physiology 99 (4), 1370-1375.

Murakami, A., 1997. Quantitative analysis of 77K fluorescence emission spectra in Synechocystis sp. PCC 6714 and Chlamydomonas reinhardtii with variable PS I/PS II stoichiometries. Photosynthesis Research 53 (2-3), 141-148.

Murakami, A., Fujita, Y., 1988. Steady state of photosynthesis in cyanobacterial photosynthetic systems before and after regulation of electron transport composition: Overall rate of photosynthesis and PSI/PS II composition. Plant and Cell Physiology 29 (2), 305-311.

Murakami, A., Fujita, Y., 1991. Regulation of photosystem stoichiometry in the photosynthetic system of the cyanophyte Synechocystis PCC 6714 in response to light-intensity. Plant and cell Physiology 32 (2), 223-230.

Murakami, A., Kim, S.-J., Fujita, Y., 1997. Changes in photosystem stoichiometry in response to environmental conditions for cell growth observed with the cyanophyte Synechocystis PCC 6714. Plant and Cell Physiology 38 (4), 392-397.

Murata, N., Takahashi, S., Nishiyama, Y., Allakhverdiev, S. I., 2007. Photoinhibition of photosystem II under environmental stress. Biochimica et Biophysica Acta (BBA)-Bioenergetics 1767 (6), 414-421. 
Nedbal, L., Tichỳ, V., Xiong, F., Grobbelaar, J. U., 1996. Microscopic green algae and cyanobacteria in high-frequency intermittent light. Journal of Applied Phycology 8 (4-5), 325-333.

Nelson, N. B., Prezelin, B. B., 1990. Chromatic light effects and physiological modeling of absorption properties of heterocapsa pygmaea (= glenodinium sp.). Marine Ecology Progress Series 63, 37-46.

Niizawa, I., Heinrich, J. M., Irazoqui, H. A., 2014. Modeling of the influence of light quality on the growth of microalgae in a laboratory scale photo-bio-reactor irradiated by arrangements of blue and red LEDs. Biochemical Engineering Journal 90, 214-223.

Norsker, N.-H., Barbosa, M. J., Vermuë, M. H., Wijffels, R. H., 2011. Microalgal production-a close look at the economics. Biotechnology Advances 29 (1), 24-27.

Orr, L., Govindjee, 2013. Photosynthesis web resources. Photosynthesis Research 115 (2-3), 179-214.

Ortigueira, M. D., 2011. Fractional Calculus for Scientists and Engineers. Vol. 84. Springer Science \& Business Media.

Osmond, C. B., 1994. What is photoinhibition? some insights from comparisons of shade and sun plants. Photoinhibition of Photosynthesis: From Molecular Mechanisms to the Field, 1-24.

Phillips Jr, J. N., Myers, J., 1954. Growth rate of Chlorella in flashing light. Plant Physiology 29 (2), 152.

Podlubny, I., 1998. Fractional differential equations: An introduction to fractional derivatives, fractional differential equations, to methods of their solution and some of their applications. Vol. 198. Academic Press.

Pollari, M., Rantamäki, S., Huokko, T., Kårlund-Marttila, A., Virjamo, V., Tyystjärvi, E., Tyystjärvi, T., 2011. Effects of deficiency and overdose of group 2 sigma factors in triple inactivation strains of Synechocystis sp. strain PCC 6803. Journal of Bacteriology 193 (1), 265-273. 
Posten, C., 2009. Design principles of photo-bioreactors for cultivation of microalgae. Engineering in Life Sciences 9 (3), 165-177.

Pottier, L., Pruvost, J., Deremetz, J., Cornet, J.-F., Legrand, J., Dussap, C., 2005. A fully predictive model for one-dimensional light attenuation by Chlamydomonas reinhardtii in a torus photobioreactor. Biotechnology and Bioengineering 91 (5), 569-582.

Preda, L., 2016. Splitting and accelerating Gaussian beam modulated by Mittag Leffler function. Optik-International Journal for Light and Electron Optics 127 (3), 1066-1070.

Preisendorfer, R. W., 1961. Application of radiative transfer theory to light measurements in the sea. International Union of Geodesy and Geophysics Monograph 10, 11-30.

Quintana, N., Van der Kooy, F., Van de Rhee, M. D., Voshol, G. P., Verpoorte, R., 2011. Renewable energy from cyanobacteria: energy production optimization by metabolic pathway engineering. Applied Microbiology and Biotechnology 91 (3), 471-490.

Rabe, A. E., Benoit, R. J., 1962. Mean light intensity-a useful concept in correlating growth rates of dense cultures of microalgae. Biotechnology and Bioengineering 4, 377-390.

Rabinowitch, E., Govindjee, 1969. Photosynthesis. John Wiley \& Sons.

Rakhimberdieva, M. G., Boichenko, V. A., Karapetyan, N. V., Stadnichuk, I. N., 2001. Interaction of phycobilisomes with photosystem II dimers and photosystem I monomers and trimers in the cyanobacterium Spirulina platensis. Biochemistry 40 (51), 15780-15788.

Richmond, A., 2003. Growth characteristics of ultrahigh-density microalgal cultures. Biotechnology and Bioprocess Engineering 8 (6), 349-353.

Richmond, A., 2008. Handbook of microalgal culture: biotechnology and applied phycology. John Wiley \& Sons. 
Richmond, A., Cheng-Wu, Z., Zarmi, Y., 2003. Efficient use of strong light for high photosynthetic productivity: interrelationships between the optical path, the optimal population density and cellgrowth inhibition. Biomolecular Engineering 20 (4-6), 229-236.

Rippka, R., Deruelles, J., Waterbury, J. B., Herdman, M., Stanier, R. Y., 1979. Generic assignments, strain histories and properties of pure cultures of cyanobacteria. Microbiology 111 (1), 1-61.

Rittmann, B. E., 2008. Opportunities for renewable bioenergy using microorganisms. Biotechnology and Bioengineering 100 (2), 203-212.

Rögner, M., Nixon, P. J., Diner, B. A., 1990. Purification and characterization of photosystem I and photosystem II core complexes from wild-type and phycocyanin-deficient strains of the cyanobacterium Synechocystis PCC 6803. Journal of Biological Chemistry 265 (11), 6189-6196.

Sarada, R., Pillai, M. G., Ravishankar, G., 1999. Phycocyanin from Spirulina sp: influence of processing of biomass on phycocyanin yield, analysis of efficacy of extraction methods and stability studies on phycocyanin. Process Biochemistry 34 (8), 795-801.

Saxena, R., Mathai, A., Haubold, H., 2002. On fractional kinetic equations. Astrophysics and Space Science 282 (1), 281-287.

Schagerl, M., Künzl, G., 2007. Chlorophyll a extraction from freshwater algae-a reevaluation. Biologia 62 (3), 270-275.

Schiessel, H., Metzler, R., Blumen, A., Nonnenmacher, T., 1995. Generalized viscoelastic models: their fractional equations with solutions. Journal of Physics A: Mathematical and General 28 (23), 6567-6584.

Schuurmans, R. M., Schuurmans, J. M., Bekker, M., Kromkamp, J. C., Matthijs, H. C., Hellingwerf, K. J., 2014. The redox potential of the plastoquinone pool of the cyanobacterium Synechocystis species strain PCC 6803 is under strict homeostatic control. Plant Physiology 165 (1), 463-475. 
Sedoud, A., López-Igual, R., ur Rehman, A., Wilson, A., Perreau, F., Boulay, C., Vass, I., Krieger-Liszkay, A., Kirilovsky, D., 2014. The cyanobacterial photoactive orange carotenoid protein is an excellent singlet oxygen quencher. The Plant Cell 26 (4), 1781-1791.

Shen, G., Boussiba, S., Vermaas, W., 1993. Synechocystis sp PCC 6803 strains lacking photosystem I and phycobilisome function. The Plant Cell 5 (12), 1853-1863.

Simis, S. G., Kauko, H. M., 2012. In vivo mass-specific absorption spectra of phycobilipigments through selective bleaching. Limnology and Oceanography: Methods 10 (4), 214-226.

Singh, A. K., Bhattacharyya-Pakrasi, M., Elvitigala, T., Ghosh, B., Aurora, R., Pakrasi, H. B., 2009. A systems-level analysis of the effects of light quality on the metabolism of a cyanobacterium. Plant Physiology 151 (3), 1596-1608.

Stamatakis, K., Tsimilli-Michael, M., Papageorgiou, G. C., 2014. On the question of the light-harvesting role of $\beta$-carotene in photosystem II and photosystem I core complexes. Plant Physiology and Biochemistry $81,121-127$.

Stramski, D., Morel, A., 1990. Optical properties of photosynthetic picoplankton in different physiological states as affected by growth irradiance. Deep Sea Research Part A. Oceanographic Research Papers $37,245-266$.

Sukenik, A., Bennett, J., Falkowski, P., 1987. Light-saturated photosynthesis-aalimitation by electron transport or carbon fixation? Biochimica et Biophysica Acta (BBA)-Bioenergetics 891 (3), 205-215.

Sung, M.-G., Shin, W.-S., Kim, W., Kwon, J.-H., Yang, J.-W., 2014. Effect of shear stress on the growth of continuous culture of Synechocystis PCC 6803 in a flat-panel photobioreactor. Korean Journal of Chemical Engineering 31, 1233-1236.

Takaichi, S., Maoka, T., Masamoto, K., 2001. Myxoxanthophyll in Synechocystis sp. PCC 6803 is myxol 2'-dimethyl-fucoside,(3 r, 2' s)-myxol 
2'-(2, 4-di-o-methyl- $\alpha$-l-fucoside), not rhamnoside. Plant and Cell Physiology 42 (7), 756-762.

Telfer, A., 2002. What is $\beta$-carotene doing in the photosystem II reaction centre? Philosophical Transactions of the Royal Society B: Biological Sciences 357 (1426), 1431-1440.

Terry, K. L., 1986. Photosynthesis in modulated light: quantitative dependence of photosynthetic enhancement on flashing rate. Biotechnology and Bioengineering 28 (7), 988-995.

Thrane, J.-E., Kyle, M., Striebel, M., Haande, S., Grung, M., Rohrlack, T., Andersen, T., 09 2015. Spectrophotometric analysis of pigments: A critical assessment of a high-throughput method for analysis of algal pigment mixtures by spectral deconvolution. PLOS ONE 10 (9), 1-24.

Tian, L., van Stokkum, I. H. M., Koehorst, R. B. M., Jongerius, A., Kirilovsky, D., van Amerongen, H., 2011. Site, rate, and mechanism of photoprotective quenching in cyanobacteria. Journal of the American Chemical Society 133 (45), 18304-18311.

Tolbert, N., 1985. Relationship of glycolate excretion to the dic pool in algae. Inorganic Carbon Uptake by Aquatic Photosynthetic Organisms, 221-227.

Touloupakis, E., Cicchi, B., Benavides, A. M. S., Torzillo, G., 2016a. Effect of high ph on growth of Synechocystis sp. PCC 6803 cultures and their contamination by golden algae (Poterioochromonas sp.). Applied Microbiology and Biotechnology 100 (3), 1333-1341.

Touloupakis, E., Cicchi, B., Torzillo, G., Sep 2015. A bioenergetic assessment of photosynthetic growth of Synechocystis sp. PCC 6803 in continuous cultures. Biotechnology for Biofuels 8 (1), 133, 11 pages.

Touloupakis, E., Rontogiannis, G., Benavides, A. M. S., Cicchi, B., Ghanotakis, D. F., Torzillo, G., 2016b. Hydrogen production by immobilized Synechocystis sp. PCC 6803. International Journal of Hydrogen Energy 41 (34), 15181-15186. 
Tramontana, V., Casasanta, G., Garra, R., Iannarelli, A. M., 2013. An application of Wright functions to the photon propagation. Journal of Quantitative Spectroscopy and Radiative Transfer 124, 45-48.

Trautmann, A., Watzer, B., Wilde, A., Forchhammer, K., Posten, C., 2016. Effect of phosphate availability on cyanophycin accumulation in Synechocystis sp. PCC 6803 and the production strain BW86. Algal Research 20, 189-196.

Tsunoyama, Y., Bernát, G., Dyczmons, N. G., Schneider, D., Rögner, M., 2009. Multiple rieske proteins enable short-and long-term light adaptation of Synechocystis sp. PCC 6803. Journal of Biological Chemistry 284 (41), 27875-27883.

Tyystjärvi, E., Aro, E.-M., 1996. The rate constant of photoinhibition, measured in lincomycin-treated leaves, is directly proportional to light intensity. Proceedings of the National Academy of Sciences 93 (5), 2213-2218.

Tyystjärvi, T., Tuominen, I., Herranen, M., Aro, E., Tyystjärvi, E., 2002. Action spectrum of psba gene transcription is similar to that of photoinhibition in Synechocystis sp. PCC 6803. FEBS Letters 516, 167-171.

Ughy, B., Ajlani, G., 2004. Phycobilisome rod mutants in Synechocystis sp. strain PCC6803. Microbiology 150 (12), 4147-4156.

Umena, Y., Kawakami, K., Shen, J.-R., Kamiya, N., 2011. Crystal structure of oxygen-evolving photosystem II at a resolution of 1.9 å. Nature 473 (7345), 55.

Vajravel, S., Kovács, L., Kis, M., Rehman, A. U., Vass, I., Gombos, Z., Toth, T. N., 2016. $\beta$-carotene influences the phycobilisome antenna of cyanobacterium Synechocystis sp. PCC 6803. Photosynthesis Research 130 (1-3), 403-415.

Van de Hulst, H. C., 1957. Light scattering by small particles. John Wiley. 
van Stokkum, I. H., Gwizdala, M., Tian, L., Snellenburg, J. J., van Grondelle, R., van Amerongen, H., Berera, R., 2018. A functional compartmental model of the Synechocystis PCC 6803 phycobilisome. Photosynthesis Research 135 (1-3), 87-102.

Vavilin, D., Brune, D. C., Vermaas, W., 2005. 15n-labeling to determine chlorophyll synthesis and degradation in Synechocystis sp. PCC 6803 strains lacking one or both photosystems. Biochimica et Biophysica Acta (BBA)-Bioenergetics 1708 (1), 91-101.

von Wobeser, E. A., Ibelings, B. W., Bok, J., Krasikov, V., Huisman, J., Matthijs, H. C., 2011. Concerted changes in gene expression and cell physiology of the cyanobacterium Synechocystis sp. strain PCC 6803 during transitions between nitrogen and light-limited growth. Plant Physiology 155 (3), 1445-1457.

Wang, L., Dai, Y., Chen, W., Shao, Y., Chen, F., 2016. Effects of light intensity and color on the biomass, extracellular red pigment, and citrinin production of Monascus ruber. Journal of Agricultural and Food Chemistry 64 (50), 9506-9514.

Wang, Q., Hall, C. L., Al-Adami, M. Z., He, Q., 2010. Isia is required for the formation of photosystem I supercomplexes and for efficient state transition in Synechocystis PCC 6803. PloS ONE 5 (5), e10432.

Warren, C., Weedon, B., 1958. Carotenoids and related compounds. Part VII. Synthesis of canthaxanthin and echinenone. Journal of the Chemical Society, 3986-3993.

Westermark, S., Steuer, R., 2016. Toward multiscale models of cyanobacterial growth: A modular approach. Frontiers in Bioengineering and Biotechnology 4, 95.

Wilhelm, C., Selmar, D., 2011. Energy dissipation is an essential mechanism to sustain the viability of plants: the physiological limits of improved photosynthesis. Journal of Plant Physiology 168 (2), 79-87.

Wozniak, B., Dera, J., Ficek, D., Majchrowski, R., Kaczmarek, S., Ostrowska, M., Koblentz-Mishke, O. I., 2000. Model of the in vivo spec- 
tral absorption of algal pigments. Part 1. Mathematical apparatus. Oceanologia 42, 177-190.

Wright, S., Jeffrey, S., Mantoura, R., 1997. Phytoplankton pigments in oceanography: guidelines to modern methods. UNESCO Publishing.

Xu, H., Vavilin, D., Funk, C., Vermaas, W., 2004. Multiple deletions of small cab-like proteins in the cyanobacterium textitSynechocystis sp. PCC 6803: consequences for pigment biosynthesis and accumulation. Journal of Biological Chemistry 279 (27), 27971-27979.

Yacobi, Y. Z., Köhler, J., Leunert, F., Gitelson, A., 2015. Phycocyaninspecific absorption coefficient: Eliminating the effect of chlorophylls absorption. Limnology and Oceanography: Methods 13 (4), 157-168.

Young, A., Frank, H., 1996. Energy transfer reactions involving carotenoids: quenching of chlorophyll fluorescence. Journal of Photochemistry and Photobiology B: Biology 36 (1), 3-15.

Yun, Y.-S., Park, J., 2001. Attenuation of monochromatic and polychromatic lights in Chlorella vulgaris suspensions. Applied Microbiology and Biotechnology 55, 765-770.

Zakar, T., Herman, E., Vajravel, S., Kovacs, L., Knoppová, J., Komenda, J., Domonkos, I., Kis, M., Gombos, Z., Laczko-Dobos, H., 2017. Lipid and carotenoid cooperation-driven adaptation to light and temperature stress in Synechocystis sp. PCC6803. Biochimica et Biophysica Acta (BBA)-Bioenergetics 1858 (5), 337-350.

Zakar, T., Laczko-Dobos, H., Toth, T. N., Gombos, Z., 2016. Carotenoids assist in cyanobacterial photosystem II assembly and function. Frontiers in Plant Science 7, 295, 7 pages.

Zaman, S. F., Baleanu, D., Petráš, I., 2016. Measurement of para-xylene diffusivity in zeolites and analyzing desorption curves using the Mittag-Leffler function. Fractional Calculus and Applied Analysis 19 (2), 551-560.

Zavřel, T., Sinetova, M. A., Búzová, D., Literáková, P., Červenỳ, J., 2015. Characterization of a model cyanobacterium Synechocystis sp. PCC 
6803 autotrophic growth in a flat-panel photobioreactor. Engineering in Life Sciences 15 (1), 122-132.

Zhang, L., Selão, T. T., Selstam, E., Norling, B., 2015. Subcellular localization of carotenoid biosynthesis in Synechocystis sp. PCC 6803. PloS ONE 10 (6), e0130904.

Zhang, W., Ren, Y., Zhang, Y., Zhang, Z., 2010. Light attenuation of Synechocystis sp. PCC 6803 in photo-bioreactor and its growth dynamics research. Journal of Anhui Agricultural Sciences 38, 99579958,9967. 